\title{
Leadership standards: Professional Development Schools versus non-Professional Development Schools
}

\author{
David L. Lancaster \\ West Virginia University
}

Follow this and additional works at: https://researchrepository.wvu.edu/etd

\section{Recommended Citation}

Lancaster, David L., "Leadership standards: Professional Development Schools versus non-Professional Development Schools" (2010). Graduate Theses, Dissertations, and Problem Reports. 4623.

https://researchrepository.wvu.edu/etd/4623

This Dissertation is protected by copyright and/or related rights. It has been brought to you by the The Research Repository @ WVU with permission from the rights-holder(s). You are free to use this Dissertation in any way that is permitted by the copyright and related rights legislation that applies to your use. For other uses you must obtain permission from the rights-holder(s) directly, unless additional rights are indicated by a Creative Commons license in the record and/ or on the work itself. This Dissertation has been accepted for inclusion in WVU Graduate Theses, Dissertations, and Problem Reports collection by an authorized administrator of The Research Repository @ WVU.

For more information, please contact researchrepository@mail.wvu.edu. 
Leadership Standards: Professional Development Schools

Versus non-Professional Development Schools

David L. Lancaster

Dissertation submitted to the College of Human Resources and Education at

West Virginia University

in partial fulfillment of the requirements

for the degree of

\author{
Doctor of Education \\ in \\ Educational Leadership Studies \\ Paul E. Chapman, Ph.D., Chair \\ Cynthia Kelley, Ph.D. \\ Ted Price, Ph.D. \\ Richard Walls, Ph.D. \\ Diane Yendol-Hoppey, Ph.D.
}

Department of Educational Leadership Studies

Morgantown, West Virginia

2010

Keywords: Principals, Professional Development Schools, non-Professional Development

Schools, Perception, ISLLC Standards

Copyright 2010 David L. Lancaster 


\section{Abstract \\ Leadership Standards: Professional Development Schools Versus non-Professional Development Schools}

David L. Lancaster

This study compares the perceptions of two groups of West Virginia school principals through a survey instrument built in alignment with the Interstate School Leaders Licensure Consortium (ISLLC) standards. The perceptions of principals affiliated with Professional Development Schools (PDS) are compared to the perceptions of principals who are not affiliated with Professional Development Schools (non-PDS). The 587 principals in the state of West Virginia with active e-mail addresses were asked to participate in an online survey. The survey instrument contained six domains with 31 items aligned with the Interstate School Leaders Licensure Consortium's (ISLLC) six national standards on leadership. Two hundred forty-nine principals responded for a return rate of $43.3 \%$. The treatment group (PDS) had 117 principals that responded, and the comparison group (non-PDS) had 132 principals that responded. Only one statistically significant event (as determined by a $t$-test) occurred within the survey's 31 items. The statistically significant difference was found in Domain 2 (Advocating): Item 2 (collaboration). 


\section{Acknowledgements}

This dissertation is dedicated to my loving family. This work would not have been possible without their love, patience, and concern. My wife Kathy has served as my friend, my confidant, and a continued source of encouragement when I believed that I would never see the light at the end of the tunnel! Her unwavering support and strength encouraged me to follow this dream. I want to thank my children, Camaron, Kyle, Connor, and Caraline for the many hours they gave up for me to follow this dream. This work is also dedicated to my mother and late father, Hilda and Leroy Lancaster who have always believed there was no mountain too high for me to climb. Oh, where would I be without my brother, Kenny Lancaster always telling me to take care of my business first? I just want to thank all of you for allowing me to achieve this dream.

I am especially grateful to my chair, Paul Chapman, for his constant support and thoughtful guidance. I would like to thank my committee members: Dr. Walls who served as my psychometrics expert, Ted Price who served as my Public School and Higher Education connection, Dr. Diane Yendol-Hoppey who served as my PDS connection with WVU and Dr. Cynthia Kelley for serving as my outside committee member.

I would also like to extend my appreciation to Carol Hysell, a dear friend and Peggy Clifford who graciously provided their time and helpful feedback in order for me to achieve my dream. I am also obliged to the principals from the state of West Virginia who participated in this study. Their generosity of time and responses made this project possible. 
Table of Contents

\begin{tabular}{|c|c|c|}
\hline \multicolumn{2}{|l|}{ Chapter One } & 1 \\
\hline & Background of the Study & 1 \\
\hline & The Problem Statement & 8 \\
\hline & Purpose and Significance of the Study & 10 \\
\hline & Overview of the Method & 13 \\
\hline & Research Questions and Hypotheses & 14 \\
\hline & Delimitations & 17 \\
\hline & Definitions of Terms & 18 \\
\hline \multicolumn{2}{|c|}{ Chapter Two: Review of Related Literature } & 21 \\
\hline & A Snapshot of Professional Development School History & 21 \\
\hline & Evolution of Leadership Preparation Programs & 38 \\
\hline & Interstate School Leaders Licensure Consortium Standards & 54 \\
\hline & Connecting Research to Practice & 61 \\
\hline & Current Research, School Leadership, and PDS & 62 \\
\hline & Dissertation Focused on Administrators' Perceptions of a Change Initiative & 66 \\
\hline & Dissertation Focused on School Based Site Coordinators view of a PDS & 67 \\
\hline & Use of Research Based Leadership Practices by PDS Principals & 71 \\
\hline & Dissertation Focused on the Essence of the Principal's Role in a PDS & 74 \\
\hline & Summary of Related Literature & 82 \\
\hline \multicolumn{2}{|c|}{ Chapter Three: Research Design } & 85 \\
\hline & Research Questions and Hypotheses & 86 \\
\hline & Participants & 90 \\
\hline & Instrument Characteristics & 91 \\
\hline & Survey Instrument Development & 91 \\
\hline & Panel of Interviewers & 93 \\
\hline
\end{tabular}


$\begin{array}{ll}\text { Data Analysis } & 96\end{array}$

\begin{tabular}{|c|c|}
\hline Survey Questionnaire Dissemination and Collection & 98 \\
\hline Summary of Research Methods & 100 \\
\hline Chapter Four: Analysis of Results & 103 \\
\hline Descriptive and Statistical Analysis by Domain & 105 \\
\hline Domain 1: Visioning (ISLLC Standard 1) & 106 \\
\hline Domain 2: Advocating (ISLLC Standard 2) & 107 \\
\hline Domain 3: Management (ISLLC Standard 3) & 109 \\
\hline Domain 4: Collaboration (ISLLC Standard 4) & 111 \\
\hline Domain 5: Ethics (ISLLC Standard 5) & 112 \\
\hline Domain 6: Community Relations (ISLLC Standard 6) & 114 \\
\hline Descriptive and Statistical Analysis by Item & 115 \\
\hline Survey Domain 1 (Visioning) & 115 \\
\hline Visioning-Item 1 & 115 \\
\hline Visioning-Item 2 & 117 \\
\hline Visioning-Item 3 & 118 \\
\hline Visioning-Item 4 & 119 \\
\hline Visioning-Item 5 & 120 \\
\hline Survey Domain 2 (Advocating) & 121 \\
\hline Advocating: Item 1 & 121 \\
\hline Advocating: Item 2 & 122 \\
\hline Advocating: Item 3 & 124 \\
\hline Advocating: Item 4 & 125 \\
\hline Advocating: Item 5 & 126 \\
\hline Advocating: Item 6 & 127 \\
\hline Advocating: Item 7 & 128 \\
\hline Advocating: Item 8 & 130 \\
\hline Advocating: Item 9 & 131 \\
\hline Survey Domain 3 (Management) & 132 \\
\hline Management: Item 1 & 132 \\
\hline
\end{tabular}


Management: Item 2

\begin{tabular}{cc}
\hline Management: Item 3 & 134 \\
\hline Management: Item 4 & 136 \\
\hline Management: Item 5 & 137 \\
\hline Survey Domain 4 (Collaboration) & 138 \\
\hline Collaboration: Item 1 & 138 \\
\hline Collaboration: Item 2 & 139 \\
\hline Collaboration: Item 3 & 140 \\
\hline Collaboration: Item 4 & 141 \\
\hline Survey Domain 5 (Ethics) & 143 \\
\hline
\end{tabular}

$\begin{array}{ll}\text { Ethics: Item } 1 & 143\end{array}$

Ethics: Item 2

\begin{tabular}{cc}
\hline Ethics: Item 3 & 145 \\
\hline Ethics: Item 4 & 146 \\
\hline Ethics: Item 5 & 148 \\
\hline Survey Domain 6 (Community Relations) & 149 \\
\hline
\end{tabular}

Community Relations: Item $1 \quad 149$

Community Relations: Item 2

Community Relations: Item $3 \quad 151$

\begin{tabular}{lr}
\hline Analysis Of Narrative Responses & 152
\end{tabular}

$\begin{array}{ll}\text { Question } 1 & 152\end{array}$

Question 2

$\begin{array}{ll}\text { Chapter Summary } & 154\end{array}$

$\begin{array}{ll}\text { Chapter Five: Discussion } & 158\end{array}$

$\begin{array}{ll}\text { Synopsis of the Study } & 158\end{array}$

\begin{tabular}{lr}
\hline Instrument Development Phase & 158 \\
\hline Instrument Domains Correlated to ISLLC Standards & 160 \\
\hline Results & 161 \\
\hline Narrative Question One & 162 \\
\hline Awareness of ISLLC Standards & 162 \\
\hline
\end{tabular}




\begin{tabular}{|c|c|c|}
\hline & Awareness of accountability & 163 \\
\hline & ISLLC Standards and Leadership & 163 \\
\hline & Narrative Question Two & 163 \\
\hline & Category: Time & 164 \\
\hline & Conclusions & 164 \\
\hline & Recommendations for Further Study & 165 \\
\hline References & & 167 \\
\hline Appendices & & 180 \\
\hline & A Principal Perception Survey & 180 \\
\hline & B Letter of Support from State Superintendent & 183 \\
\hline & C Principal Advance E-mail & 184 \\
\hline & D Participation Request (Letter of Support) & 185 \\
\hline & E Follow up E-mail 1 & 186 \\
\hline & F Follow up E-mail 2 & 187 \\
\hline & G Follow up E-mail 3 & 188 \\
\hline & H Narrative Question 1(non-PDS) & 189 \\
\hline & I Narrative Question 1 (PDS) & 192 \\
\hline & J Narrative Question 2 (non-PDS) & 194 \\
\hline & K Narrative Question 2 (PDS) & 195 \\
\hline & L Commonalities in Standards ISLLC/NCATE & 204 \\
\hline
\end{tabular}




\section{Chapter One}

The focus of this study is how Professional Development School (PDS) principals perceive their professional world according to the Interstate School Leaders Licensure Consortium (ISLLC) national standards for school leaders as compared to non-Professional Development School principals. This study is based on the development of an instrument to assess professional development school principals' perceptions about their leadership in accordance with the ISLLC standards, referred to as, "The Principal Perception Survey (see Appendix A).

Chapter One of the proposal presents the background for this study, specifies the problem statement of the study, describes the significance of the study, provides an overview of the method, and presents the research questions. The chapter concludes by noting the delimitations of the study and defining specific terms.

Chapter Two is a review of related literature. It describes the problem in a current frame of reference, as well as describing historical perspectives of PDS, leadership preparation, the history of the ISLLC standards, and recent dissertations associated with related topics. Finally, Chapter Two will tie the problem statement, significance of the study, and the research question presented in Chapter One to the rest of the study.

Chapter Three presents the methodology used in the study. This includes an explanation of the design of the study, the survey or sampling procedures, the data collection and related safeguard management procedures, and the data analysis procedures.

\section{Background of the Study}

The primary stakeholders of American education are students and their families. Never before have external and internal forces in education taken on such urgency for stakeholder 
groups. The external influences are the globalizing world, which puts pressure on the American system and causes many to question whether our students are being prepared to compete in an international economy. The internal factors students and parents are concerned about are the quality of instruction, the relevancy of curriculum content, and visionary leadership for $21^{\text {st }}$ century challenges. It is not new for the public to insist on school reform, but the prevailing conditions bring about a new urgency.

Quality of instruction for American schools was a major issue in 1983. In April of 1983, A Nation at Risk was presented as an open letter to the public and the Secretary of Education by the National Commission on Excellence in Education. T. H. Bell created the National Commission on Excellence in Education. He directed the committee to examine the quality of education in the United States. In April of 1983, the group published their findings in, A Nation at Risk: the Imperative for Education Reform. The report emphasized a responsibility to provide leadership and assistance to schools and universities. A Nation at Risk serves as a reminder that change is a constant process in the educational system. Without change, the educational system in the United States will not progress, as do the educational systems in other countries. A majority of principals today was not in leadership positions when this report became public; however, the need for change in the school system remains a common concern for today's society. The American public seeks reform in the current educational system in all areas. According to A Nation at Risk, the educational foundations of our society are slipping away. America's tradition of being a leader in education has always stood without question. The corrosion, caused by mediocrity, threatens our current educational system and the future of the United States. This groundbreaking report signaled a "unilateral educational disarmament" 
within the American educational system (The National Commission on Excellence in Education, 1983 p. 1).

Following the call for educational reform after the report, A Nation at Risk, other information emerged calling for continued change in education. In 1987, the Carnegie Task Force on Education and the Economy presented A Nation Prepared calling for change (Carnegie Forum on Education and the Economy, 1987). Another report issued by the Holmes group, tomorrow's Schools in 1990, contained information from a group of the nation's leading deans in Colleges of Education (The Holmes Group, 1990). Throughout, the reports call for examination and change in the systems of teacher education across the country. These reports led to a continued call for change within our school systems. Quality of instruction remains the focus of school reform efforts.

President George W. Bush continued the pursuit for quality of instruction through change by legislative means. The No Child Left Behind (NCLB) agenda focuses on creating a mastery level of skills for all students. The testing process of NCLB determines whether a student knows the required content or can do the required skill as outlined in the state's standards. A non-partisan group was present on January 8, 2002 when President George W. Bush signed the No Child Left Behind Education Bill. President Bush thanked Senator Edward Kennedy, George Miller, Steve Chabot, and all those that had made NCLB possible. The leadership of the country felt the education system needed a change to protect America's future. President Bush stated, "We owe the children of America a good education, and today begins a new era, a new time in public education in our country. As of this hour, America's schools will be on a new path of reform and a new path of results" (Bush, $2002 \mathrm{pg}$. 2). The passage of the No Child Left Behind Act has turned up the heat even more in the demand for quality 
instruction. The full weight of the federal policy is in place and it demands accountability from our schools.

The relevancy of curriculum content is a major concern in our schools because of the competition in an international economy. A recent book by Thomas Friedman titled "The World is Flat" expressed the current educational needs of the twenty-first century. Workers must upgrade their education and knowledge skills so they can occupy one of the new high skill jobs that are being created in the expanding markets. America, as a whole, will do fine with free trade provided it continues to churn out knowledgeable workers who can produce ideas and goods that can compete in the new global markets. Educational institutions will need to produce highly skilled workers to enable American society to claim a healthy slice of the world market. Friedman explains how the flattening of the world is occurring. The world started to flatten at the start of the twenty-first century. The age of information and communication is located at an individual's fingertips with the Internet connections of today. With the flattening of the globe, individuals are required to run faster in order to stay in their current competitive place. These circumstances have implications for countries, companies, communities, and individuals. Governments, societies, and individuals must adapt in order to survive (Friedman, 2005). A picture of what children need to learn is coming into focus. Challenges to achieve learning and evaluation continue into the $21^{\text {st }}$ century (Pearlman, 2006). The stake- holders in America's educational system are concerned about the relevant content of the curriculum in schools today.

Friedman observed the American school system's need for relevant content on an international level. While in Bangalore, he observed the young, highly educated Indians working at engineering jobs at a fraction of the wages Americans make. This type of young digital native represents the real competition of a flattening world. Companies of today have multi-national 
boards that are concerned with cost and quality only. The multi-national boards are not concerned with saving a small town in Texas. Their main interest is profit. This international type of highly skilled individual is what maintains the pressure on the need for change in the American education system of today. Without highly skilled students entering the work force, America will lose its competitive advantage in a globalized economy. The prior example describing the global competitive workforce maintains that curriculum must be relevant to the skills needed today in an international market (Friedman, 2007).

The next issue parents are concerned about is the leadership necessary to keep the quality of instruction high and the relevance of curriculum content on target. Parents want to know if the leaders in our school systems are adequate for $21^{\text {st }}$ century challenges.

Arthur Levine completed a four- year study on the preparedness of school leaders. The report concluded the weakest programs in education lay within the administration branch of professional development. The number of strong leadership programs is limited, and most or those programs are evaluated as weak or appalling. Much of this is a result of inadequate clinical instruction, weak faculties, insufficient financial support, and poor research. The report suggests advancement should occur through a short series of certificate programs geared to the needs of today's leaders. However, Levine remains optimistic that school leadership programs can change and better prepare the school leaders of tomorrow. The awareness of the problems concerning school leadership is growing within the educational community. Levine cites correspondence with individuals throughout the educational community upon completion of his report. For example, Arthur Wise, the president of the National Council for Accreditation of Teacher Education, agreed with the analysis and most of the recommendations in the report 'Education School Leaders' (Levine, 2005). The National Council for the Accreditation of 
Teacher Education (NCATE) responded to the call for leadership accountability within schools by producing the Curriculum Guidelines for school administrators. These guidelines help to ensure quality of instruction in the classroom, the relevance of curriculum content for a highly skilled workforce, and the type of leadership needed for $21^{\text {st }}$ century challenges. The quality of leadership within our schools remains a theme in the school reform movement of the $21^{\text {st }}$ century.

The Interstate School Leaders Licensure Consortium (ISLLC) on November 2, 1996 adopted the first set of universal standards for school leaders. The ISLLC team believed that the standards approach provided the best avenue to assure parents that they have the right to expect quality leadership for the $21^{\text {st }}$ century. Another aspect of the document was to continue dialogue about quality educational leadership among stakeholders in the area of school administration (Interstate School Leaders Licensure Consortium, 1996).

During this challenging time, many educators have started to support an initiative referred to as a Professional Development School (PDS) concept. A Professional Development School is set in a state of simultaneous renewal for students, teachers, and principals (Goodlad et al. 1990). The climate is one of reflection and correction to achieve a relevant skill. Between the years of 1995-2001, the National Council for Accreditation of Teacher Education (NCATE) worked with hundreds of teacher educators to design standards for a professional development school. These standards addressed many areas such as quality of instruction, the relevance of curriculum content, and leadership for $21^{\text {st }}$ century challenges. Lee Teitle, (2003) articulated the type of theoretical goals of a Professional Development School for students:

The purpose of professional development schools is to promote student learning. PDSs do that by improving schools, preparing new teachers in better ways, supporting the 
growth and development of all educators, and using inquiry and research to see what is working well and what is not. Given the wide gap in achievement among students of differing racial, ethnic, and economic backgrounds in this country, PDSs have a special interest in promoting the learning of all students and reducing the achievement gap. ( $\mathrm{p}$. Xvii)

Information on how Professional Development Schools affect the quality of instruction, the relevance of curriculum content, and leadership for the $21^{\text {st }}$ century in our schools are areas of interest to the stakeholders of American education.

Districts around the country are reporting that pools of principal candidates are drying up. By looking at the abundance of responsibilities and the complexity of decisions leaders are asked to make each day, we can begin to see the multitude of reasons for the current problems existing in the educational system relative to leadership. The school leaders of the twenty-first century must change their method of operations and adapt in order to survive. School leaders must have a clear understanding of the best pedagogical practices and be able to transfer that to what teachers do in the classroom to provide quality instruction for students. The principal must be able to recognize the difference between relevant and non-relevant curriculum and take steps to eliminate non-relevant curriculum. The principal must deal with constant change within and without the school system and be ready to encompass the $21^{\text {st }}$ century. The conditions of school leadership are difficult. Many principals may receive endorsements, but few feel supported. Some say they are not prepared to take on these demanding leadership positions given the current condition of schools (Keller, 2000). 


\section{The Problem Statement}

This research focuses on the problem of school leadership conditions that drive current leaders away and thwart the ambitions of emerging school leaders. One of the main areas of concern is the shortage of qualified individuals willing to assume the responsibilities of the principal position. Many believe that administrators have been overburdened. Principal preparation programs have failed to plan for the next generation of school leaders. Given these dynamics, there is a shortage of qualified principals today (Murphy, 2005). The principal is the key player in the direction a school system will follow (Hammonds, 2002). Other individuals referred to the principal as the gatekeeper of change. We can no longer afford to lose quality school leaders in such tenuous times. The future looks bleak for all individuals involved in the school system of today if current trends involving school leaders do not change. Stakeholders must find a way to stop the trend of losing qualified principals and find a way to make the school leadership position attractive to emerging leaders (Fullan, 2004).

The overburdened principal of today requires assistant principals, and heads of various departments to assist in fulfilling their plethora of tasks. Even with the close support of assistants and department heads, the principal's multi-tasking has become burdensome. Some type of support structure beyond assistant principals and department heads must be in place for the principal to achieve the array of responsibilities expected of today's leader. If a supporting environment for the leadership is not in place, then all educators fail (Pellicer \& Anderson, 1995). The principals must deal with many complex issues such as, organizational changes, curriculum, instruction, pupil management, personnel management, school community relationships, and new skills in technology. All of the prior issues have expanded to the point that no one person alone can stand without some type of supportive structure. Without an 
encouraging atmosphere the principals find themselves feeling overburdened and in a state of helplessness.

Many criticize the failure to plan for the next generation of leaders as a reason for the leadership shortage. Mike Milstein and Associates (1993) studied principal-preparation programs for the Danforth Foundation and believed that universities have hoarded the programs and not really collaborated with school districts to stay current with leadership issues of today or the future. The current preparation process for individuals aspiring to be principals is viewed as one of the reasons for the leadership shortage. The lack of training leaves the principal feeling inadequate and overwhelmed. Keller felt principals were the key to a school's success and questioned why principal preparation was so unsatisfactory. Keller felt principal preparations were unsatisfactory and "about as wrong-headed as expecting someone to whip up a meal for a hungry crowd on the basis of some courses in economics and chemistry" (Keller, 2000, p. 1)

It is more likely a principal will stay in their current position of leadership if he or she is not overwhelmed and/or overburdened. The Professional Development School structure intends to build or create a community of support for all participants. Within the PDS structure, the continual renewal of teaching and learning of all participants is the key to self-actualization. Professional Development Schools are places where a principal will seek a self-actualized work environment. Maslow viewed self-actualization as self-fulfillment, the realization of personal potential (Woolfell, 2007). School leaders should want to understand themselves professionally. This type of understanding allows principals to do a better job tomorrow than today, due to reflection.

A plan must be in place to train the next generation of leaders. The PDS culture fosters the development of new leaders. The type of reflection practiced within a PDS may be the 
answer to developing self-actualized leaders. During the course of this study, the research will focus on the perceptions of school principals. The hypothesis related to this study is leaders within a PDS have a positive perception of their work because of the founding principles for the PDS movement. The founding PDS principles are as follows: professional preparation of candidates, faculty development, improvement of practice through inquiry, and enhanced student learning (National Council for Accreditation of Teacher Education, 2001). The founding principles of the PDS movement are built on creating an entire teaching and learning community based on democratic values. The PDS structure uses the full range of knowledge and skills of classroom teachers, school administrators, future teachers, and professors to shape the school (Richardson Foundation, 1990).

\section{Purpose and Significance of the Study}

How do we determine if the PDS system is providing the support structure for current and future principals to grow professionally as they lead? First, we must determine if a relationship exists between a principal's participation in a professional development school and his or her perception of the various facets of his or her leadership roles.

To research this question, an assessment tool was designed. It will be referred to as The Principal Perception Survey. The survey utilized the six ISLLC standards in its design.

The ISLLC standards are the foundation for nationally recognized programs that produce school leaders. If the PDS culture truly fulfills its primary intent to foster professional selfactualization, then it follows that principals from the PDS culture will have strong beliefs and perceptions that align with the ISLLC standards.

The purpose of this study is to develop an instrument that focuses on the perceptions of PDS principals in alignment with the Interstate School Leaders Licensure Consortium (ISLLC) 
standards. This study will add professional knowledge to the base of information already present concerning the PDS culture. Principals' perceptions from this study will gauge what a PDS culture looks like from their point of view. During the course of this study, an instrument will be constructed using the perceptions of principals in alignment with ISLLC standards to take a snapshot of how supportive the PDS climate is for them.

The question to be addressed in this study is, Do Professional Development School leaders perceive their work to be in alignment with the ISLLC standards? The PDS culture, by its nature, is supposed to foster professional growth for all of the professionals involved in the teaching and learning process.

The professionals in PDS schools are expected to engage in the process of personal and professional reflection to ensure professional growth. It is the PDS culture that evolves from all those working in this process of personal and professional reflection that enhances the ability of the organization to achieve its goals and maintain a consistent state of organizational renewal. These areas involve changes in relationships among a list of stakeholders such as teachers, pupils, parents, and principals (Murphy, 2005). Central to any PDS is the professional development of educators and administrators (Teitel, 2003). This study will provide information on the nature of the PDS culture in accordance with the ISLLC standards through principals' perceptions.

The Interstate School Leaders Licensure Consortium (ISLLC) national standards intent was to provide a hard target for quality educational leadership. The ISLLC standards present a common core of knowledge, dispositions, and performances that link leadership to productive schools and enhanced educational outcomes (Murphy, Shipman, 1999). The ISLLC standards 
are what every good principal should know about, value about their work, and be able to do in modeling for school leadership.

This study will take a precise look at how principals perceive their work in two different leadership settings: Professional Development Schools (PDS) versus non-Professional Development Schools. Through this type of study on educational leadership, a better understanding about good leadership in accordance with the ISLLC standards will be gained as perceived by principals. The information derived from the study on PDS and non-PDS principals will help to gauge whether PDS principals and non-PDS principals understand and model good leadership as outlined by the ISLLC standards.

Given the structure of the PDS, educators involved in that teaching and learning community should have a clear understanding of best practices. Making these best practices a part of everyday operations, gives everyone a common understanding of good school leadership. The consistency between what PDS leaders think, do, and say about their work should correlate with the ISLLC best leadership practices. If a type of correlation exists between PDS and the ISLLC standards then perhaps a new training ground could exist for principals. Possibly a Professional Development School could be a cradle for the development of principals. When comparing the Interstate School Leaders Licensure (ISLLC) standards to the National Council Accreditation for Accreditation of Teacher Education (NCATE) some commonalities exist. Some key words and concepts flow through both sets of standards.

The ISLLC standards do not directly correlate with the NCATE standards. The ISLLC Standards, which are designed for an organization (professional development school partners), have a different focus than the ISLLC standards, which are designed for individuals (school 
administrators). Nevertheless, we would expect both organizations to share key values and concepts, and they do.

The NCATE standards and the ISLLC standards both emphasize the concept of a learning community and both organizations address the primacy of community in their first standard. Each organization has a standard that directly addresses the need for collaboration of the shareholders within that community. Both organizations recognize the need for the management of resources and structures; however, NCATE uses a different vocabulary emphasizing "accountability and quality assurance."

NCATE's fourth standard directly addresses "Equity and Diversity." The ISLLC standards use a different approach. The ISLLC Standards use the phrase "the success of all students" in each standard. The fifth ISLLC standard directly addresses ethics, while the NCATE standards focus on accountability. Please see Appendix L.

\section{Overview of the Method}

School districts around the country are reporting that pools of principal candidates are drying up while the pressures on schools to do a better job of educating their students continue to build. As new accountability measures take effect, a broad consensus has emerged in education policy circles that raising the quality of school leadership is essential (Keller et al., 2000). West Virginia, like other states, has individuals certified to hold the position of principal: however, only a limited number of individuals are willing to take on the role of leadership. Principals continue to retire, and fewer and fewer qualified individuals apply for the leadership positions. There continues to be a great demand for qualified, energized, and professional school leaders.

"The study of principles which undergird sustained educational innovations in both of the spheres of influence which teacher education embraces-the schools and the universities-is an 
important related sub-topic to examine" (Hubbard, 2003, p. 9). The central focus of this research is developing an instrument to measure the impact of professional development school cultures on professional development school principals' perceptions of their work as it is related to the ISLLC standards. A systematic data collection will be useful to provide evidence that the standards are being employed in a variety of way (Murphy \& Shipman, 1999). An instrument developed to see how school leaders perceive their work within a PDS school culture in relationship to the ISLLC standards will be a worthy study.

\section{Research Questions and Hypotheses}

Does the PDS relationship affect how leaders perceive their work in accordance with the ISLLC standards? For the purposes of this study, the focus will be on the Interstate School Leaders Licensure Consortium standards (ISLLC) covering six domains.

Domain 1: An educational leader promotes the success of every student by facilitating the development, articulation, implementation, and stewardship of a vision of learning shared and supported by all stakeholders.

$\mathrm{H}_{0}$ : There is no statistically significant difference in Domain $\underline{1}$ scores when comparing individuals who are principals of Professional Development Schools with those who are principals of non-Professional Development Schools.

$\mathrm{H}_{1}$; There is a statistically significant difference in Domain $\underline{1}$ scores when comparing individuals who are principals of Professional Development Schools with those who are principals of non-Professional Development Schools.

Domain 2: An educational leader promotes the success of every student by advocating, nurturing, and sustaining a school culture and instructional program conducive to student learning and staff professional growth. 
$\mathrm{H}_{0}$ : There is no statistically significant difference in Domain $\underline{2}$ scores when comparing individuals who are principals of Professional Development Schools with those who are principals of non-Professional Development Schools.

$\mathrm{H}_{1}$ : There is a statistically significant difference in Domain $\underline{2}$ scores when comparing individuals who are principals of Professional Development Schools with those who are principals of non-Professional Development Schools.

Domain 3: An educational leader promotes the success of every student by ensuring management of the organization, operations, and resources for a safe, efficient, and effective learning environment.

$\mathrm{H}_{0:}$ There is no statistically significant difference in Domain $\underline{3}$ scores when comparing individuals who are principals of Professional Development Schools with those who are principals of non-Professional Development Schools.

$\mathrm{H}_{1}$ : There is a statistically significant difference in Domain $\underline{3}$ scores when comparing individuals who are principals of Professional Development Schools with those who are principals of non-Professional Development Schools.

Domain 4: An educational leader promotes the success of every student by collaborating with families and community members, responding to diverse community interests and needs, and mobilizing community resources.

$\mathrm{H}_{0:}$ There is no statistically significant difference in Domain $\underline{4}$ scores when comparing individuals who are principals of Professional Development Schools with those who are principals of non-Professional Development Schools. 
$\mathrm{H}_{1}$ : There is a statistically significant difference in Domain $\underline{4}$ scores when comparing individuals who are principals of Professional Development Schools with those who are principals of non-Professional Development Schools.

Domain 5: An educational leader promotes the success of every student by acting with integrity, fairness, and in an ethical manner.

$\mathrm{H}_{0}$ : There is no statistically significant difference in Domain $\underline{5}$ scores when comparing individuals who are principals of Professional Development Schools with those who are principals of non-Professional Development Schools.

$\mathrm{H}_{1}$ : There is a statistically significant difference in Domain $\underline{5}$ scores when comparing individuals who are principals of Professional Development Schools with those who are principals of non-Professional Development Schools.

Domain 6: An educational leader promotes the success of every student by understanding the profile of the community, and responding to and influencing the larger political, social, economic, legal, and cultural context.

$\mathrm{H}_{0}$ : There is no statistically significant difference in Domain $\underline{6}$ scores when comparing individuals who are principals of Professional Development Schools with those who are principals of non-Professional Development Schools.

$\mathrm{H}_{1:}$ There is a statistically significant difference in Domain $\underline{6}$ scores when comparing individuals who are principals of Professional Development Schools with those who are principals of non-Professional Development Schools. 


\section{Delimitations}

Classifying or defining a Professional Development School is a difficult target. Book (1996) observed, "Researchers and teacher educators are often at a loss to define when a school is actually a Professional Development School. Is it when the university and school district label it a PDS or make a commitment to create one?" (p. 204). "As more PDSs have developed, even though there has been some variability in the ways in which various authors define the concept and its purposes, there has been a convergence of agreement about operational terms and standards" (Hubbard, 2003, p. 9). The structure of the Professional Development School or non-Professional Development School will not have any bearing on the selection process of schools in this study; each professional development school and non-Professional Development School has a unique structure. Therefore, results will reflect a diversity of perceptions in relation to the efficiency of the Professional Development School and non-Professional Development School.

The question remains, when does a school actually become a Professional Development School? "As more PDSs have developed, even though there has been some variability in the ways in which various authors define the concept and its purposes, there has been a convergence of agreement about operational terms and standards" (Hubbard, 2003, p. 9). Just because principal or superintendent inks a formal agreement does not guarantee that, a school follows the true standards of a PDS. However, this study uses a Formal Partnership agreement to determine PDS status.

The results of this study do not be apply on a national level. This study will use a convenient sampling of West Virginia principals with active e-mail addresses. Results from this study will only reflect the opinions of principals who are willing to participate in the study. 


\section{Definitions of Terms}

The terms mentioned in brief are the operational definitions that will be used for this study.

Content validity - the degree to which a test measures an intended content area; it is determined by expert judgment and requires both item validity and sampling validity (Gay, Mills, \& Airasian, 2006).

Dimension - clusters of related indicators. (Vogt, 2005).

Holmes Group - Comprising 100 major research universities committed to reforming teacher education and the teaching profession: the Holmes Group has issued three reports of national interest in the two areas (The Holmes Group, 1990).

Interstate School Leaders Licensure Consortium (ISLLC) - was created under the auspices of the Council of Chief State School Officer and in cooperation with the National Policy Board for Educational Administration. ISLLC set out to develop a powerful framework for redefining school leadership and to marshal the forces necessary to bring that design to life. One of the results of the consortium's work was the creation of the ISLLC standards (Murphy \& Shipman, 1999).

Interstate School Leaders Licensure Consortium (ISLLC) standards - forged from research on productive educational leadership and the wisdom of colleagues, the standards were drafted by personnel from 24 state education agencies and representatives from various professional associations. The standards present a common core of knowledge, dispositions, and performances that will help link leadership more forcefully to productive schools and enhanced educational outcomes (Interstate School Leaders Licensure Consortium, 2006).

Mean- the arithmetic average of a set of scores (Gay, Mills, \& Airasian, 2006). 
National Council for Accreditation of Teacher Education (NCATE) - is one of the accreditation bodies for Professional Development Schools.

Null Hypothesis $\left(\mathrm{H}_{0}\right)$ an hypothesis that a researcher usually hopes to reject, thereby substantiating its opposite. The "null" does not necessarily refer to zero or no difference (although it usually does); rather it refers to the hypothesis to be nullified or rejected (Vogt, 2005).

Professional Development School (PDS) - innovative institutions formed through partnerships between professional education programs and P-12 schools. Their mission is professional preparation of candidates, faculty development, inquiry directed at the improvement of practice, and enhanced student learning (National Council for Accreditation of Teacher Education 2001).

Psychometric Research - research on how psychological variables are operational for purposes of measurement, particularly measurement of individual differences among people (Vogt, 2005).

Reform - refers to planned and unplanned changes. Many times these changes have acknowledged goals and predetermined implementation plans (Salaiz, 2004)

SPSS - (Statistical Package for the Social Sciences) The SPSS is a widely used brand of computer software that performs most standard statistical analyses of data.

Statistical Coding - translating data from one language or format into another. The coding schemes are decided before data is collected. The data is defined and measured (Vogt, 2005).

In the following chapter, selected pertinent research will be reviewed as a foundation from which the study grows. Chapter Two is a review of the history of professional 
development schools, leadership preparation, the ISLLC standards, recent dissertations associated with related topics, and a review of how research came to the social sciences. 


\section{Chapter Two: Review of the Related Literature}

\section{A Snapshot of Professional Development School History}

The related research is broken into four domains. Domain One focuses on a general overview of the history of Professional Development Schools. Domain Two focuses on how leadership preparation has evolved. Domain Three focuses on the emergence of the ISLLC standards. Domain Four focuses on connecting research to practice. Domain five focuses on recent dissertations associated with related topics.

The National Council for Accreditation of Teacher Education (NCATE) defines what a Professional Development School is and what standards must be maintained to receive its endorsement. The organization of NCATE is one of the accreditation bodies for Professional Development Schools. Professional Development Schools (PDSs) are innovative institutions formed through partnerships between professional education programs and Pre K-12 schools. Professional Development Schools are usually exemplary functioning schools in a public school setting with a four-fold mission. The mission includes the preparation of new teachers, faculty development, pedagogical improvement through reflective practice, and the enhancement of overall student achievement and success (NCATE, 2001).

“The PDS is historically linked to John Dewey's laboratory school, a place where pedagogy was to be developed according to scientific strategies of exhibition, testing, verification, and theory development" (Hubbard, 2003, p. 9). Dewey had many influences in his life in developing different educational concepts. For example, Dewey studied the Greeks' experiments in education completed over 2,500 years ago. His favorite person to read about during his career, was the philosopher and educator, Plato, (Breault and Breault, 2005). During 
his reading and study, Dewey concluded that learning is an active process. He believed the term education represented questioning, inquiring, and judging (Dewey, 1920).

Concepts resembling the structure of professional development schools started during the Progressive Era with John Dewey. John Dewey lived from 1859-1952. He was an American philosopher, psychologist, and educational reformer whose thoughts and ideas have been greatly influential in the United States and around the world. Upon Dewey's death in 1952, he was memorialized as America's most famous philosopher. He was revered by liberal educators and deplored by conservatives but universally acknowledged as the intellectual voice for America (Ryan, 1995). Although there are a number of progressive philosophers such as Jean-Jacque Rousseau, Leo Tolstoy, Johann Pestalozzi, Maria Montessori, Friedrich Froebel, C.S. Peirce, and others, John Dewey has been mentioned as the leader of the Progressive Movement more than any other educators (Perrone, 1989). Dewey conceived the idea of teachers being "students of teaching" who would reflect on their own practice while learning from another educator (Teitel, 2003).

According to Dewey, the child must be involved in an active process in order to learn, and needs to process information in a familiar or working environment. Education should not be broken into unrelated disciplines of study. In many cases, the curriculum becomes an obstacle rather than a support for the child. When the child faces the curriculum that is unrelated to his or her world the facts are torn away from their original place in experience and rearranged with reference to some general principle. This means the child must view facts objectively without reference to their place and meaning in the child's world. Children learn best with the experiences that promote their own well-being, or that of his or her family and friends. Development in its truest form is connected with experience the student seeks (Dewey, 1920). 
During the Progressive Era, while Dewey was discovering the fundamental principles of the PDS, the term did not yet exist. Dewey's idea was that children would come to school in a community that would provide real, guided experiences, thereby fostering their capacity to contribute to society. For example, Dewey believed students should be involved in real-life tasks and challenges. He referred to this type of learning as active learning. Active learning was never meant to let children do whatever they want and follow whatever grabs their attention at the moment. Dewey believed the task of the educator was to engage students actively so they could obtain skills, which produced immediate satisfaction in their work. Teachers or educators also benefited from the experience of being involved with students in the active learning process. Teachers gained skills and knowledge that they otherwise would not have had the opportunity to learn through reflection. In addition, teachers gained confidence in themselves and their teaching abilities through practice. These core beliefs were practiced at a progressive laboratory school. It was also referred to as a settlement house at the University of Chicago. The formation of the lab school in Chicago serves as a landmark in America School's history (Breault and Breault, 2005).

Jane Addams was born in 1860 and died in 1935. Addams' work embodied the Progressive Education Movement of the time. During this time in American history, child labor was a topic of debate. Addams fought for children's rights and stood against the exploitation of children. Addams' concern about the dangers of child labor propelled her into the political arena. She helped to form the National Child Labor Committee in 1904 (Schugurensky, 2002). Jane Addams and Ellen Starr founded a settlement house in the slums of Chicago in 1889. The settlement house concept was designed to be implemented in the most needy and poorest communities to aid in the success of the students and the settlement house concept. The 
settlement house was called Hull House. The house was at one time an elegant brick mansion built in 1856 by Charles J. Hull, a real-estate developer. However, by 1889 the Hull House was located in the heart of a poor immigrant Chicago neighborhood. The immigrant population in 1889 represented three-quarters of the city's one million inhabitants. In a short time, the Hull House became a complex of thirteen buildings. The complex included an art gallery, gymnasium, theater, dining hall, playground, and even apartments for staff. The Hull House attracted countless numbers of immigrants. Estimates indicate that approximately two thousand people entered the doors of the Hull House each week (Schugurensky, 2002).

John Dewey served on the Board of Directors for the Addams' Hull House.

Occasionally, he lived in the staff complex and worked at the Hull House. His educational philosophy of moving away from subject-centered rote exercises toward a cooperative, childcentered exploration of interests was put into practice in the Hull House setting. Dewey felt Addams was a personification of his educational idea that people learn by doing. Later, upon Addams' death, Dewey would dedicate one of his books to her Liberalism and Social Action (Schugurensky, 2002).

Addams believed that the emerging social consciousness would lead to improved education for children, which would alleviate the suffering of the poor. She recognized that charity was not the long-term answer for the poor, and she believed that both the rich and the poor could learn from one another. In addition, Addams believed that the same educational opportunities should be available to all. The Hull House was a success project during a difficult economic time in American history. When the depression of the nineteen thirties struck, Addams saw many of the things she had advocated and fought for become policies under 
President Franklin Roosevelt. She received numerous awards and became the first American woman to receive the Nobel Peace Prize in 1931 (Smith, 2000).

Lucy Sprague Mitchell's ideas coincided with Dewey’s and the Progressive movement. Mitchell used "schools of inquiry" to further the progressive ideas of the era (Lyons et al., 1997). Over Mitchell's lifetime from 1878-1967, she witnessed a variety of changes in America and the structure of education. Between 1890 and 1920, the United States experienced many changes. Industrialization of America was occurring with the movement of the general population from farms to cities. The increase in immigration accompanied a rise in poverty. Such conditions laid the groundwork for social and educational reforms. Mitchell was a teenager when she met Dewey. Dewey influenced Mitchell's ideas, which influenced her later work. Despite little experience with underprivileged people, she studied Jane Addams' work at the Chicago school (Smith, 2000).

Other reform movements were occurring during the early nineteen hundreds. In 1908 Henry Pritchett, president of the Carnegie Foundation, believed strongly that medical schools were in need of immediate reform. Pritchett believed training for young doctors could be considered haphazard. At this particular time in the medical community, there were no standards for practice. Pritchett decided to produce a survey to determine the condition of medical schools in the United States. He chose schoolmaster and educational theorist, Abraham Flexner, to head the survey. Over the course of eighteen months, Flexner visited all 155 medical schools within the United States. The report produced by Flexner indicated a great gulf between the best and worst medical schools concerning requirements for the students and performance indicators. Flexner indicated in his report that it was time to develop a number of properly supported institutions and the speedy demise of all inferior medical schools. Abraham Flexner's advocacy 
of the teaching hospital was built on Dewey's idea of actively engaging students to obtain needed medical skills. Abraham Flexner's model of a teaching hospital created a reform movement in the medical community (Beck 2004).

The fight for school reform continued during Dewey's lifetime. Many individuals joined Dewey in the fight for school reform. On February 21, 1917 at the "Learning for Earning" annual meeting: John Dewey, Samuel Gompers, President of the American Federation of Labor, and Owen R. Lovejoy, Secretary of the National Child Labor Committee, called for vocational training to be put in place in public schools. They recommended that the funding for this reform come from the public sector to keep public schools free from industrial exploitation. Dewey explained that the three R's are not good enough for the industrial workers needed for 1917 and the future work force of America. He believed the education process needed to be modified to produce students ready to handle modern industrial machinery, rapid transportation, and to be involved in a competitive market (New York Times, 1917).

Samuel Gompers supported Dewey's call for reform in public education to produce workers ready to use the modern machinery of the day. Samuel Gompers was the first and longest-serving president of the American Federation of Labor (AFL). The high point of the AFL's and Gompers political strategy occurred during President Woodrow Wilson's administration from 1912-1920. During World War I, Gompers was appointed to the Council of National Defense (AFL-CIO website). However, on February 21, 1917 Gompers not only called for school reform with Dewey, but also insisted that the funding for public education come from public funds. Gompers believed that public schools nourish the foundational supports of democracy. He believed that if funding came from any other source, it would breed suspicion and give curriculum control to private interests. If philanthropic friends of education desire to 
provide funding, let them arrange a way for the public agents to be given control of the money (New York Times, 1917).

From the twenties through the fifties, Lucy Sprague Mitchell continued the reform movement in education. An example of a unique innovative effort occurred in 1931. Mitchell opened the Cooperative School for Student Teachers, later known as the Cooperative School for Teachers (CST). Throughout the twenties, the majority of teachers in the United States merely had high school degrees. Mitchell and the CST joined forces with eight experimental schools in order to create a new breed of teacher. Mitchell wanted a trained individual who could bring a practical attitude to each teaching situation, and help future teachers develop a scientific attitude toward their work (Smith, 2000).

The development of Dewey and Mitchell's concepts can be traced through the Bureau of Educational Experiments and Bank Street College (Mitchell, 1950). The teachers' documentation, records, and reflections serve as important sources of knowledge about the learning environment. The original concepts of Dewey concerning how students learn and in what structure they learn best received continued support from the contemporary works of Nathan and Susan Isaacs, Jerome Brunner, John Holt, Bill Hull, Joseph Featherstone, and James Herndon (Perrone, 1989). Additional support from noted theorist Jean Piaget and Eleanor Duckworth extended Dewey's concepts into a more recent era in education. Piaget's (18961980) work on the cognitive development of children coincided with Dewey's principles on student learning in many areas. According to Piaget, the student's role shifts from passive to active learning when they view teachers as collaborators in specific areas of expertise. Dewey and Piaget viewed learning as being holistic and inseparable from the social and historical realities of the student's daily experiences (Breault and Breault, 2005). 
Prior research conducted by Eleanor Duckworth, a professor of education at Harvard Graduate School of Education, and a translator and a former student Jean Piaget, defined the conditions under which learning takes place. She asserts children will stop having their own thoughts if their ideas are not valued by adults. The devaluation of student's ideas forces him or her into rote memorization. This process destroys the student's exploration and creativity. Duckworth's explanation of how a student learns reflects Dewey's education philosophy of moving away from subject-centered rote exercises toward a cooperative, child-centered exploration of interests. Dewey's philosophy still lies at the heart of many bold educational experiments, such as Outward Bound. Dewey helped to shape Abraham Flexner's advocacy of the teaching hospital in the reform of medical education (Levine, 1992).

Teaching hospitals emerged at a critical time in the history of the medical profession and the history of the hospital as an institution. Public education was facing the same kind of challenge the medical profession faced earlier in the same century. The training for teachers needed change, as well as the teacher training schools themselves. Albert Shanker, president of the American Federation of Teachers (1964-1984), led the AFT in designing an institution referred to as a professional practice school. Shanker used the information produced by the Holmes Group to emphasize the need for school change. The Exxon Education Foundation was a major financial contributor in the school restructuring process. Many projects sprang up with a variety of names such as; training schools, clinical schools, professional practice schools, and professional development schools (Levine, 1992).

Professional development schools are compared to the teaching hospital model advocated by Abraham Flexner. A PDS is considered the equivalent to the teaching hospitals in the medical profession. The intent of the PDS is to provide opportunities to improve professional 
education by providing necessary links between schools and universities (Holmes Group, 1986). The reason for the comparison is that teaching hospitals like professional development schools provide clinical preparation for student teachers much like teaching hospitals do for medical students and interns. Going beyond the comparison between teaching hospitals and PDSs, the future intent of the PDS structure is to redesign teacher preparation and transform the teaching profession (Darling-Hammond, 1994). This education reform movement has come full circle with the use of Dewey's ideas in the development of Abraham Flexner's model of a teaching hospital to medicine as the PDS is to education (Levine, 1992).

During the sixties, Schager (1967) referred to schools as Centers of Inquiry. The Centers of Inquiry maintain a connection to institutions of higher learning in a formal structure. The practice in "Centers of Inquiry" included participation in advanced graduate courses, research, and jointly conceived investigations between public school faculty and higher education faculty. The sharing of knowledge enhanced the professional development between both institutions. The collaboration benefited both the public school and the university. The university would lend sufficient support to enable the public school to investigate its own problems. In return, the public school would serve as an additional source for research. During the collaboration between the two institutions, common ground was found between the two faculties. In addition, common terms and definitions of the type of jobs began to appear. Schager (1967) observed, "In time, perhaps, schools organized as centers of inquiry would become comparable to teaching hospitals in the medical profession" (p. 75).

The Professional Development School concept has been in place in a variety of forms and concepts for some time. However, the term professional development school has not always been the term to identify a particular type of school design. The evolutionary course to the term 
professional development school started with the ideas of the progressive movement with Dewey in the lead. Next, the structures for clinical preparation for student teachers much like teaching hospitals do for medical students and interns created a type of working model. This working model was copied from Abraham Flexner's teaching hospital model; however, no particular name or label was applied in the initial stages.

Terms such as key schools, clinical schools, centers of inquiry, laboratory schools, training schools, professional practice schools, and professional development schools started to emerge to identify the working model. Individuals organizing the Holmes Group met at the University of Wisconsin. John Palmer, education dean at Wisconsin, lead the discussion on strong professional schools for educators. During this discussion, the different names of professional schools were discussed. The group felt clinical schools suggested people going to a clinic and not portraying students. Key schools did not pose any type of mindset for the structure of a professional school. Laboratory schools sounded too experimental and carried baggage of early lab schools. This group coined the term "professional development school" due to no history in the name, and it seemed to serve the intent of the professional school structure (Darling-Hammond, 1994).

Another example of how the name evolved through time involved Marsha Levine. In 1992, she used the professional practice school term to describe the modern structure we refer to today as professional development schools. Today, Levine serves as Senior Consultant for the National Council for Accreditation of Teacher Education and refers to the same concept and working model as a professional development school. The standards being used in most states to determine the quality of a PDS was created by the National Council for Accreditation of Teacher 
Education in 1996. It is interesting to note that the concept of a PDS is perceived to be important enough to be included as a positive institutional indicator by NCATE.

The Professional Development School structure has been on an evolutionary course like its name due to many calls for reform and reports issued demanding change. One of those reports that brought concern to the education system was a report titled, A Nation at Risk in the early nineteen eighties (Klaumeier, 1990). The report was the result of eighteen months of study and indicated that reform needed to occur within the educational system in fundamental areas.

The eroding public opinion of the educational system forced the system's response: the PDS. The public perceives the universities as being unwilling to link themselves to school reform issues that taxpayers care about (Frazier, 1994). Also involved is the financial support for school reform. Finding funding for the PDS projects has been a continuing challenge. The funding comes from different areas. The majority of professional development schools started out with little funding. The PDS movement has been encouraged by The Holmes Group, The National Network for Educational Renewal, The American Association of Colleges for Teacher Education, The National Education Association, and The American Federation of Teachers (Darling-Hammond, 1994). Public schools and universities have worked together in the past. Laboratory schools and curriculum reform of the nineteen sixties brought the two institutions together.

The PDS, however, departs from the past in two directions. First, the PDS is an effort to invent an institutional coalition that will bring all the required forces togetheruniversities, schools of education, and public schools, and second, it promises to work on the problems of teaching over the long haul (The Holmes Group, 1990, p. 2). 
An example of the call for reform being turned into action occurred in West Virginia. In the mid-nineteen eighties, West Virginia became one of the first states in the country to establish a PDS partnership through the Benedum Collaborative. The Benedum (1985) project followed the PDS structure creating "a place where public schools and universities integrate professional practice in schools with professional preparation programs in colleges and universities to enhance the quality of both. "There are thirty-one professional development schools within five counties associated with the Benedum Collaborative" (p. 1). Richard Hawthorne, the Dean of the College of Education at West Virginia University during the start of the Benedum Collaborative explained the kinds of profound and complex changes needed to sustain PDS relationships:

The changes expected in the faculty and culture of teacher education program is very much the same as those reform expectations laid out for schools. That is, the expectation represents the substantive rethinking and redirecting of core beliefs, constructs, and roles that dominate the way things have been done in Colleges of Education for decades (Hawthorne, 1997, p. 296).

In addition, Exxon and Ford corporations supported the professional development school movement. In 1988, Exxon supported an initiative by the American Federation of Teachers. A task force was set up to answer questions on how to prepare teachers for restructured schools. The goal of this project was to envision what a restructured school and the method of delivery would look like. During this project, Professional Development Schools were referred to as Professional Practice Schools (Levine, 1992).

Another report issued in 1990 by the Holmes Group called for the leading schools of education to train teachers the way medical schools use hospitals to train doctors. The 
expectation would be that teacher candidates serve as classroom observers and interns in the school: both to learn and practice their skills. Teachers would work with teacher candidates to plan lessons. The teacher candidate would have the opportunity to observe a teacher's exemplary instruction and receive the opportunity to teach lessons.

The goal of the Holmes Group was to create an agenda that would reform education. The central focus of The Holmes Group would be "professional development schools" (DarlingHammond, 1994). Part of their research indicated teachers needed to be more involved with schools. This same group, in 1986, called for vigorous new certification tests for teachers. The group envisioned a national network of "professional developments schools." This design would include public schools where university professors and students of education would work with teachers and administrators. The outcome would be new curriculum design, research projects, new teacher training, and the rejuvenation of experienced teachers.

The idea of professional development schools was not presented as new due to several variants of professional development schools already in existence. The report's contributions were the definition of common principles for such schools and a pledge to establish them in major research universities and to establish a nationwide network. The model for a professional development school would be a teaching hospital where there would be a long-term relationship between a university and a public school. This would allow both institutions to make changes in curriculum and practices to train teachers better. The proposal for PDSs recognized that efforts to reform teacher education must be accompanied by efforts to make schools better places for teachers to teach and learn. The idea is to integrate theory and practice (Holmes Group, 1990). 
The call for reform continued in 1995 with the release of Tomorrow's Schools of Education by the Holmes Group. The Holmes Group (1995) articulated the value of universitybased education schools:

Institutions preparing educators should either adopt reforms along the lines proposed in these pages or surrender their franchise... We assume this drastic stance precisely because we believe that the country needs university-based education schools and that they can make a difference in the teaching and learning of children... Schools of education that exercise introspection and focus their mission accordingly can, indeed, must help solve the problems that afflict the public school... Within these education schools and the universities that house them resides a vast, mostly untapped potential that can be unleashed in the service of better public education if only the faculty members and administrators implement change of the sort we envision... developing sensitize strategies, making sound contributions and setting stands of accountability (p. 3). Sewall, Shapiro, Ducett, and Sanford (1995) describe the PDS approach as a logical coalition building between schools and universities. A major issue facing public schools is the low public esteem experienced by schools and teacher preparation institutions. The building of a coalition between public schools and universities is a defensive reaction to the public's perceptions of schools and teacher preparation institutions. However, the invention of such new institutions creates many challenges. The growth of a PDS faces pitfalls such as "turfism," tradition, scarce resources for change, inexperience, and uncertain environmental constraints. Whose knowledge counts, and who will lead the PDS? As the profession of teaching and the PDS structure strives to mature, it must grapple with all of these issues (Darling-Hammond, 1994). Another institutional factor that appeared to hinder the growth and professionalism of 
PDSs was the change of university PDS faculty. These changes often caused disruption to educational initiatives that had begun with prior faculty from the university at a particular PDS (Kess, 2002).

The Professional Development School movement of today receives kudos from the public, state departments of education, and corporations, but the purse strings remain tied. No major foundation has made a long-term commitment specifically to the PDS movement. Federal or state funding is committed for only short periods. Institutional support for teacher education is generally less supported by universities than are most other programs. This leads to a lack of funds on which to build a labor-intensive innovation like a PDS project. Individual schools or districts have few resources to invest in a PDS project. Many schools hiring novice teachers realize that they will only prepare the new teacher to move onto a more affluent school. The majority of new hires occur in less affluent schools where resources are limited. Due to limited funding, the teacher turnover is the highest, and the cycle of novice teachers continues in the less affluent school (Teitel, 2003).

Money is needed if the PDS movement is to survive and continue the work with current action-research initiatives that address school improvement plans and pre-service teacher education (Kess, 2002). It is difficult to find a money source that will continue over an extended period. With financial resources being so limited, someone or some organization would have to make a decision to move resources from a current project. What project is going to lose capital? This is a difficult question in an educational arena already documented as having limited resources. College presidents, local superintendents, teachers' association, or a local school board would have to make a difficult decision to move funding from a current area presently receiving money. Key people would have to take a stand in those groups to make a lasting 
funding source for a PDS movement. This would only occur if key individuals were knowledgeable about a PDS and its benefits to the education community. Future hope for funding sources for PDS might be found in the states. If PDSs can become institutionalized then perhaps states will begin to acknowledge them as part of the infrastructure for a strong educational system. If this occurs, the states will fund them through basic aid allocations as they do other parts of the educational system. Another future financial source for the PDS movement might be located at the federal level. Since the federal government invested in teaching hospitals as part of its efforts to improve the medical education and ensure qualified health professionals, it is possible the federal government will invest in the PDS movement to improve education and ensure qualified teaching professionals (Darling-Hammond, 1994).

According to Lee Teitel (2003), many professional development schools got their start in funding under the umbrella for school reform. The professional development schools make sense to the business world. The on-the-job training and application is supported throughout the business world. Exxon and Ford foundations have supported education enhancement throughout the years. In 2007, the Exxon Mobile Corporation directed 69.7 million to education worldwide (Worldwide Giving Report, 2007). The leaders of funding believe that bringing multiple players to the table at the same time will provide a structure for change and better understanding. In this case, the world of business, public schools, and higher educational institutions will have a better chance of creating change by working together.

Professional Development Schools are a model for change and are still in a developmental stage (Metcalf-Turner, 1999). As such, each site will operate in a distinctive manner. Each PDS will have a distinct institutional history, mission, program design, and a variety of availability resources. Metcalf-Turner (1999) explained the diversity of PDSs: 
The PDS label does not seem as important as the work that goes on between those who are responsible for carrying out collaborative activities aimed at meeting goals for both academic and professional development. The flexibility in designing collaborative partnership between public schools and universities seemed to be one of the unique characteristics of the PDS model. Only time will tell along with further inquiry into the impact that the work has on teacher preparation professional development and student achievement (Metcalf-Turner, 1999, p. 40)

The literature related directly to Professional Development Schools is growing at a rapid pace. In 1990, Stallings and Kowalski felt that PDS projects would increase rapidly and become available for research attempting to define the PDS degree of effectiveness (Stallings \& Kowalski, 1990). In 1994, there were more than 100 PDS sites across the country (DarlingHammond, 1994). One year later, Teitel used the ERIC database and located over 200 references discussing some aspect of the PDS movement (Teitel, 1996).

Another example of the rapid growth of the PDS movement can be identified in a three part national survey conducted by the American Association of Colleges of Teacher Education (AACTE). The first survey in 1991-92 indicated that there were 28 PDS partnerships between schools and colleges or universities. Eighty elementary and secondary participating schools were identified in the 1991-92 survey. The second survey in 1993-94 indicated that 84 PDS partnerships had been established with 344 elementary and secondary schools participating in the partnerships. The final survey in $1997-98$ found more than $46 \%$ of teacher preparation programs indicated commitment to over 600 PDS partnerships (Abdal-Haqq, 1998).

The future of education is reliant on leadership of collaborative change initiatives (Fullan, 1991). Robinson and Darling-Hammond (1994) view PDS projects as "opportunities for joining 
practitioners in public schools and universities in preparing and admitting future members to their profession who are willing and able to engage in the kinds of personal and organizational development demanded by learning organizations" (p. 217).

The original concepts resembling the structure of Professional Development Schools started during the Progressive Era with John Dewey. Each Professional Development School has continued to evolve "organizationally in different ways, given the unique culture, history, and interpretation of the goals in relation to locally identified strengths and challenges" (Hubbard, 2003, p. 34). The PDS initiative, as a whole, has progressed into the $21^{\text {st }}$ century with changes to its name, rapid growth in numbers, and ever-shifting purpose. The organizational and governance structures of a PDS will continue to transform in the world of business, public schools, higher education, teachers, pupils, parents, and principals (Murphy, 1990). As a result, the emphasis on what a PDS looks like relies heavily on how respective institutions assess the changes in organizational culture, curricula, and patterns of inquiry. This will create the unique Professional Development School in each community.

\section{Evolution of Leadership Preparation Programs}

Domain 2 is focused on the preparation of the modern day school principal. School Principal is rarely the term used for the leader of today's school. The title is simply, "Principal." The principal in some situations is referred to as Head of School, Head Master, School Leader, Principal Teacher, and Chief Administrator. Many of these prior terms are used in the private school sector. The term most commonly used to describe the leader of the school is Principal. The principal is the educator who has executive authority for a school and is identified as the critical ingredient in all stages of PDS work (Foster, Loving, \& Shumate, 2000). In addition, "PDS Principals are not only facilitators of their own staff members' learning, but also of their 
roles as mentors and guides for others" (Teitel, 2003, p. 70). "So the principal's role as a supporter and promoter of interactive professionalism is essential" (Fullan \& Hargreaves, 1996 p. 84). Today, "The job of school leader has been transformed by extraordinary economics, demographic, technological, and global change" (Levine, 2005a, p. 11). The principal of today works in an era of high-stakes testing and increased accountability. The principal must answer to The No Child Left Behind Act of 2001, school boards, and the community they work in (Field, 2008).

"Our nation faces the challenge of retooling current principals and superintendents while preparing a new generation of school leaders to take their places (Levine, 2005a, p. 5). Stronger administrative leadership is needed to support the institutional mission and to maintain the vitality within the faculty (Clark \& Lewis, 1988). On July $4^{\text {th }}, 1960$ John F. Kennedy stated, "It is time for a new generation of leadership, to cope with new problems and new opportunities. For there is a new world to be won." Kennedy's statement was on target in 1960 and could be applied to the times of educational leadership today. Hess (2003) expressed the struggled to find the leadership needed in today's schools:

As the nation's schools struggle to meet the needs of ill-served children, rise to the challenge of the No Child Left Behind Act, and adjust to a world of accountability and growing competition, educational leaders face unprecedented challenges. Yet, we retain a system of recruitment, preparation, and induction that does not recruit the leaders we need, does not prepare them for their positions, does not reward them on par with their responsibilities, and locks out candidates with vital knowledge and experience. (p. 4) 
The role of the modern day principal evolved from four major periods of the last century. The four periods of time are: Ideological Era; Prescriptive Era; Behavioral Science Era; and the Dialectic Era (Murphy, 1998).

The first era of the principal evolution process defined by Joseph Murphy was the Ideological Era, 1820-1899. There is a long history of principals, but in the early days the position of principal was not viewed as an essentinal part of school operation. The term adminstrator was used in place of the term principal in many of the early conversations referring to principals. The actual number of principals was small until after the Civil War. Early schools were simple organizations, and principals were expected to learn the job through trial-and-error. Formal training for the position of principal was minimal at best. During the Ideological Era principal requirements matched those designed for teachers (Gregg, 1969).

One of the first books written about school administration in the United States was written by William L. Payne in 1875 titled, Chapters on School Supervision. Payne also taught the first college level course in school administration at Michigan in 1879 (Callahan \& Button 1964). However, educational leadership programs for school administration were unknown until the early 1900 's. The earliest formal training dealt with the ideal education needed for students and roles of the principals within the school. No courses, credits, or requirements were in place to apply for a license as a school principal before 1900.

Teachings of the Ideological Era were limited to what a principal should be and instruction within the school. The first teachings for adminstrators were theories about being exemplary school leaders. This line of teaching went into the area of leaders having certain traits that would result in great leadership. Some of this teaching was referred to as "great man" and 
"trait" theories. The remaining instruction for school adminstrators focused on curriculum and instruction (Callahan \& Button 1964).

The final analysis of the earliest period (1820-1899) for educational administration was that a principal was not recognized as a distinct position. In this earliest period, "Ideological Era," school leaders were simply authorities whose insights and on-the-job experiences provided guidance to teachers, students, and the public. Little training was required.

The second era of the principal evolution process defined by Murphy (1998) was the Prescriptive Era, 1900-1946. In the 1900's, no institutions were offering programs of study in the area of school management. Following World War II, business administration remained minor areas of study. Business schools were institutions of modest reputation viewed as intellectually step-cousins to economics departments. As the centrality of management grew in the post-War economy, executive quality increase and business schools responded to competitive forces (Hess, 2003). Meanwhile, at the end of World War II, 125 institutions were offering programs in the area of preparing school leaders (Silver, 1982). More and more principals and superintendents started their career path with university training in the practice of school adminstration during the Prescriptive Era. Many states were certifying graduates of school management programs and requiring formal training for adminstrative positions.

During the first years of the $20^{\text {th }}$ century the superintendency first grew to include elements of business managements as well as educational oversight. The modern principalship emerged at the latter part of the Prescriptive Era. "The fields of educational administration were launched by Elwood Cubberley in the twenties.” (Hess, 2003). 9).

Higher education faculty involved with the preparation of principals during the Prescriptive Era were drawn from the superintendency. Retired school administrators constituted 
the bulk of the faculty at universities with principal preparation programs. Their concept of training was to share their professional experiences under certain situations they encountered as adminstrators (National Society for the Study of Education, 1964). The idea of school leaders, as business managers, first surfaced during the early 1900s (Gregg, 1960). These individuals carried heavy teaching loads with little emphasis placed on research. Students involved in the early leadership preparation were characterized as persons serving as school administrators while attending school on a part-time basis. Faculty and students during this period were typically white males (Campbell RF, Fleming T, Newell L \& Bennion JW, 1987).

The education received by principals and superintendents during the early 1900s' was for the most part the same training teachers received. Principal and teacher training remained the same until approximately 1910-1915, at which time the corporate world's wide acceptance of scientific management changed everything. For the next 20 years the business community exerted a great deal of influence over preparation programs for school administrators (Gregg 1969).

A change in the focus of principal training occurred during the Precriptive Era (19151946). The original focus of the people's perception of what a principal should be shifted to an understanding to an understanding of the adminstrator's responsibility. The student within the principal preparation program was now studying the job of administration, its roles and how to perform these roles successfully (Campbell RF, Fleming T, Newell L \& Bennion JW, 1987).

The Faculty of the leadership preparation during this time equipped future principals for the position of the day and did not consider tomorrow. Many stories or experiences were presented to the future principals as a source of tried approaches. Almost no attention was given to challenges that were to be faced in the future by principals or theoretical concepts needed for 
potential growth in leadership. Murphy (1992) articulated the type of theoretical study during the Prescriptive Era:

The scholarship that informed course content throughout this era was little more than 'naked empiricism' or 'factualism', resulting in the development of: 'fuzzy concepts'; 'inadequately field-tested principles'; and a mere 'encyclopedia of facts' that lacked 'the power of unifying interpretive theories'. The knowledge base was comprised of: 'folklore, testimonials of reputedly successful administrators, ... the speculation of college professors'; 'personal success stories and lively anecdotes'; 'personal accounts or "war stories", and prescriptions offered by experienced practitioners'; 'experiences of practicing administrators as they managed the various problem areas of school administration'; 'maxims, exhortations, and several innocuous variations on the theme of the Golden Rule'; and 'preachments to administrators about ways in which they should perform'. (p. 31-32)

The final analysis of Prescriptive Era (1900-1946) saw the establishment of formal leadership programs, most of which emphasized technical skills, with a strong flavoring of business efficiency. During the Prescriptive Era, professors working with the leadership preparation came mostly from the superintendents. The Faculty of the Prescriptive Era attempted to prepare candidates for the principal ship, as it existed, not as it might be.

The third era of the principal evolution process defined by Murphy was the Scientific Era, 1947-1985. The Scientific Era gained support for change because of the harsh attacks on the status quo in the area of administrative training during the end of the Prescriptive Era. A considerable amount of criticism leveled against the status quo was in alignment with the personal success stories and untested principles that constituted the knowledge base of 
educational administration at the time. These types of values were inappropriate in a scientific world. During these times of change, unscientific and non-theoretical approaches to adminstration were under attack. The time for change was at hand, and the alternative vision by the Scientific Era held promise of dramatically improving the education for prospective school leaders (Murphy 1998). Scientists, not business people, held center stage during this time period. With this type of backdrop the quest for an insertion of scientific training was undertaken (Culbertson 1965). Murphy alludes to four major events that marked the Scientific Era. The first major event occurred in 1947 with the formation of the National Conference of Professors of Educational Administration (NCPEA). This organization linked together higher education faculty who worked with leadership candidates throughout the country. The NCPEA exercised considerable influence over emerging concepts entering the field of leadership. This organization also had influence over the professionals working in the school administration training programs (Campbell RF, Fleming T, Newell L \& Bennion JW, 1987).

The second defining moment for the Scientific Era occurred with the creation of the Cooperative Project in Educational Administration (CPEA). This project consisted of a consortium of eight universities funded by the Kellogg Foundation with the primary purpose of instituting changes in preparation programs. Continuing initiatives were carried through and charted during NCPEA meetings. In 1954, the focus was to encourage a multidisciplinary approach to analyze the programs used to educate school leaders (Gregg, 1969).

The third defining moment in the Scientific Era occurred in 1955 with the establishment of the Committee for the Advancement of School Administration (CASA). The work on the development of professional standards of performance turned out to be the most important work the CASA organization created. 
Finally, the fourth and final defining moment of the Scientific Era occurred in1956 with the creation of the University Council for Educational Administration (UCEA). The UCEA became a dominant force in shaping what educational administration programs studied during the 1960s and 1970s (Campbell RF, Fleming T, Newell L \& Bennion JW, 1987).

During the 1970s and 80s critics started to question and attack principals and superintendents as being out of step with public concerns and school quality. Murphy and Hallinger (1987) note,

Practitioners have become disillusioned by the failure of university programs to ground training procedures in the realities of the workplace and by their reluctance to treat content viewed as useful by administrators. This disenchantment, in turn, is partially fueling the demand for changes in methods of training school administrators. (p. 252) Training of school leaders was the target of the criticism. The low standards and the weak programs were graduating too many unprepared administrators. The criticisms prompted many states to boost licensure requirements (Hess, 2003).

Under the pull of the aforementioned forces a period of great growth occurred in the business of preparing school leaders. In 1946, approximately 125 institutions were in the industry of preparing school leaders. Forty years later, over 500 institutions were involved in the production of school leaders (National Commission on Excellence in Educational Administration, 1987).

By the eighties, principals gave birth to the notion of instructional leadership. Hess maintained the term instructional leadership "referred to school leaders who supported a culture focused on the core business of teaching and learning, provide professional development, use data to evaluate performance" (Hess, 2003, p. 9). The report, A Nation at Risk published in 
1983 continued the pressure for change within the leadership of schools. "The reform movement put a spotlight on school leadership, highlighted its importance for school success, made student achievement the measure of school performance, and demanded accountability from leaders for results (Levine, 2005a, p 17).

The faculty members of leadership programs changed during the mid 1940s to the mid 1980s. A faculty member in the 1940 s was most likely to be a generalist. Information presented to future students preparing to be principals was drawn from experience and practice of the leadership profession. By the mid 1980s, during the Scientific Era, the typical faculty member in educational administration would likely be a discipline-focused specialist with little or no practical experience. American society had become more ethnically, culturally and lingustically diverse. Many leadership positions were now filled by women. The students within the educational program became more diverse from 1940 to 1980 . The number of women and the representaion of minorities increased over the forty- year span. Murphy found that students representing the bottom quartile on national entrance exams were heavily represented within educational administration programs (Murphy, 1992).

During the scientific era, the social sciences infused the content of the preparation programs. The base of information was the study of the science of administration. The intent of this movement was to produce hypothetical and deductive knowledge in future principals to replace the seat-of-the-pants information that principals were using from the Prescriptive Era. (Crowson \& McPherson 1987). By the end of the Scientific Era, an indicator of a high quality program was the acceptance of a heavy reliance on social science content (Miklos,1983).

The final analysis of the scientific era (1947-1985) was a period of time in which theoretical ideas from the social sciences began to take precedence over seat-of-the-pants advice. 
Simultaneously the makeup of faculty changed with the old practice-oriented generalists being replaced by discipline-focused specialists with little practical experience. With enough objective data, they believed school leadership could be reshaped in a rational way.

The fourth era of the principal evolution process defined by Joseph Murphy was the Dialectic Era, 1986-present. Just like the two proceeding eras, the change to the Dialectic Era is being fueled by attacks on the current state of preparation programs. "The reputation of school leadership programs had declined sufficiently that critics could credibly suggest scrapping those programs altogether and replacing them with a variety of alternatives" (Levine, 2005a, p. 18). The assault on preparation programs seems to be harsher and more comprehensive than the earlier calls to reform in prior eras. The evidence being used to call for reform in the preparation programs comes from critiques of existing training programs (Hallinger \& Murphy 1991).

In 1987, concerns about educational leadership prompted the University Council for Educational Administration (UCEA) to form a panel to address issues involving school leadership. The National Council on Excellence in Educational Administration (NCEEA) recommended changes in the leadership arena. Areas of reform recommended by the NCEEA included reducing the number of preparation programs, increasing professional development, and reforming licensure standards (Hess, 2003).

There is a consensus in the educational community about the deficiencies of current preparation programs for future leaders. One issue is the lack of experience in the P-12 setting by higher education faculty (McCarthy \& Kuh, 1997). Another issue during the Dialectic Era involves what vision to follow. One vision suggests greater attention to matters of practice in the design and delivery of educational experiences. Another vision suggests movement toward a professional school model. Others believe the vision should start with an additional emphasis on 
values, social context, core technology, and new forms of leadership. "Think of the vision as an iceberg, the vast majority of which is underwater. Many leaders take shortcuts by slicing off the visible part of the iceberg and then assume that they have captured its full power" (Fullan, Bertani, \& Daigle, 2004, p. 2). The leaders have two points of emphasis to deliberate. One is to determine the vision to follow, and the second is to recognize the entire scope the idea represents.

An array of reports coming from a variety of stakeholders has called for reform throughout the leadership preparation programs. The National Commission on Excellence in Educational Administration (NCEEA) sponsored research on the status of the leadership preparation programs. The NCEEA report in 1987 titled Leaders for America's Schools helped crystallize the sense of what is wrong with the profession and discussed possible solutions. One of the suggestions from this report recommended the creation of a national policy board.

Directors from ten different groups with deep interest in educational leadership worked together to form the National Policy Board of Educational Administration (NPBEA) in 1988. One year later in 1989, the NPBEA released its first report. The report outlined extensive changes needed to strengthen the preparation programs. Its recommendations were adopted in a slightly modified form by the UCEA.

In the middle of all of the reports and debates, two national efforts to redefine the knowledge base in the field of leadership occurred. The National Commission for the Principalship (NCP) in 1990 published a report entitled Principals for Our Changing Schools: Preparation and Certification (National Commission for the Principalship, 1990). This report was an attempt to unpack the functional knowledge base required by principals. In addition, the NCP assigned a working team to review 21 functional domains listed in the report. This effort 
resulted in a document published in 1993 by the team titled the National Commission for the Principalship. One year later the UCEA approved six writing teams to update the knowledge bases in educational administration preparation programs (Murphy, 1998).

The Danforth Foundation was viewed as a player in the Dialectic Era for program preparation reform (McCarthy, 1998). The Danforth Foundation of St. Louis, Missouri, has a long history of influencing education. In the spring of 1985, the foundation initiated a review of literature. The results found little was being written to this point about leadership of schools. The terms superintendent and principal were rarely used. This lack of recognition in the reform literature about leadership became an area of interest for the group. At that time, the group decided to set in motion activities to emphasize the need for leadership in reform and to provide capable school leaders for the future (Milstein \& Associates, 1993).

Danforth has supported four significant efforts to assist educational administrators to selfanalyses and create change. Danforth started in the late nineteen eighties by supporting a Principals' Program to improve preparation programs for prospective leaders. This decision led to the creation of Danforth Programs for the Preparation of School Principals (DPPSP). This project began in 1987 with four universities and expanded to include 22 universities in 1992. Much information was learned because of these experimental programs. Two studies were initiated to survey basic information about the program's efforts. The results from the first study were sent to unit heads of preparation programs throughout the country. The second study consisted of case analyses of five preparation programs that were part of the Danforth project. Bruce Barnett of Northern Colorado University, Donn Gresso of East Tennessee University and David Parks of Virginia Tech made preliminary site visits, and played key roles in getting a sense of the situation in each institution and served at regional coordinators. "Subsequently, the 
three regional coordinators met with Peter Wilson, who is responsible for the foundation's DPPSP, and Mike Milstein of the University of New Mexico, who later conducted the case studies, to agree on the five programs to include in the study." (Milstein \& Associates, 1993).

Next, Danforth supported a Professors' Program to enhance the capability of departments to respond to needed reforms. The third initiative supported by Danforth was to support research and development efforts, such as the Problem-Based Learning Project. Finally, Danforth supported a series of conferences and workshops created to help simplify or introduce reform ideas for tomorrow's schools (Murphy, 1998). Milstein and Associates (1993) noted:

The experimental programs being tested at universities in cooperation with the Danforth Foundation are leading to better understandings of what is required to improve the preparation of educational administrators, and particularly of school principals. What began as a limited project in 1987 at four universities has grown to become a nationally important activity that has directly involved 22 universities, and less directly, many others have become interested observers of the effort. (p. 178)

During the Dialectic Era, two activities involving the standards pertaining to leadership were shaping the landscape. The first initiative involved the National Council for the Accreditation of Teacher Education (NCATE) setting guidelines for school administration. This project was completed over a three-year period by combining various reports and professional associations into a comprehensive framework to reshape preparation programs for school leaders. The second initiative involved the Interstate School Leaders Licensure Consortium (ISLLC), which produced the first universal set of standards for school leaders in 1996. By 2002, the ISLLC standards had been incorporated into policy by some 35 states (Hess, 2003). One of the intents for the development of the ISLLC standards was to stimulate dialogue about 
the quality of leadership across the educational landscape. The second intent was to strengthen preparation programs primarily through the manipulation of state controls over areas such as program approval (Thomson, 1998).

The current Dialectic Era (1986-present) was characterized by highly critical evaluations of administrator preparation programs and persistent efforts to transform the profession. Murphy (2006) has argued "that much of university preparation of educational leaders is, at best, of questionable value and, at worst, harmful” (p. 6). Levine (2005a) wrote “...the curriculum seemed little more than a grab bag of survey courses. If the class on the principal-ship were removed, it would be a real challenge to guess the purpose of the program." (p. 28).

The disapproval of administrator preparation programs extends to the criteria of acceptance for students entering the programs of leadership. "Educational administration applicants are among the lowest performers on standardized tests.” (Levine, 2005a) (31). Creighton and Jones in their experiences with university preparation programs have yet to witness a rejection of a candidate at the master's level requesting entrance into an educational leadership program. Creighton found it interesting that the heavy emphasis placed on the quality of program without the same importance placed on the quality of the leadership candidates. "Why is it assumed that high quality effective administrators can be produced by university programs without first attracting and selecting candidates with existing potential of school administration and leadership?” (Creighton \& Jones, 2001, p. 30).

Another issue for leadership candidates is the demand put on their time. A majority of students hold jobs and many are in present leadership positions. These types of positions demand a great deal of time and energy. Even the competent students tend to produce poor work. Leadership students are so busy at their day jobs that they have little time to devote to 
their graduate programs. "Even clinical experience tends to be squeezed in while students work full time and generally occurs in the school where the student is employed. For the most part, students described the experience as "something to be gotten out of the way" (Levine, 2005a, p. 40).

In some instances, the university treats the leadership program like a cash cow. "When university administrators use education schools as cash cows or systematically underfund them, they are in essence acknowledging that the program is weak or unimportant to them" (Levine, 2005a , p. 64). "The majority of programs range from inadequate to appalling, even at some of the country's leading universities" (Levine, 2005a, p. 23). Many times, admission standards are set to ensure quantity rather than quality. Preparation programs should aim at admitting only those who can meet high academic standards as well as demonstrate a strong commitment to meeting the needs of young people (Clark, 1998). "A growing number of education schools are lowering admission standards, watering down programs, and offering quickie degrees" (Levine, 2005a, p.24). "Until all involved parties seriously address the selection of candidates with strong analytical ability, high administrative potential, and demonstrated success in teaching, the education profession will continue to be a refuge for mediocre candidates" (Creighton \& Jones, 2001, p. 31).

Leaders of professional organizations have voiced concerns over the pool of good leadership candidates being shallow. For example, Paul Houston, executive director of the American Association of School Administrators in 2002 expressed his concern over the limited number of good leadership candidates. Hess noted recent sentiment among superintendents found fewer than 40 percent were happy with their principals' ability to make tough decisions. While filling a principal position, 60 percent of superintendents felt they had to take whatever 
they could get. The problem is not a lack of warm bodies. The individuals applying for leadership positions need the skills, training, and knowledge to lead $21^{\text {st }}$ century schools (Hess, 2003).

Some of the criticism has led to revisions and evaluations of programs for school principals. Partly because of the evaluation of formal preparation involving school principals, many formal programs have a clearer vision for the entry-level for school administrators (Leithwood, Louis Seashore, Anderson, \& Wahlstrom, 2004). In addition, there has been better communication among the diverse groups interested in the preparation of school leaders, and a notable effort to define rigorous standards for the profession.

No longer can the principal simply monitor the instructional process by setting curricular goals, monitoring lesson plans, and evaluating teachers. Today, instructional leaders immerse themselves in the technology of teaching and learning. The data must be used to make decisions and align staff development with student learning needs. The Education Commission of the States, in analyzing how the No Child Left Behind Act will affect leaders, noted principals needed a sophisticated understanding of assessment, and they should be master teachers so assessment data could be used intelligently (Anthes, 2002).

The current period is difficult to see clearly, and we are far from certain what future historians will say about it. The majority of the education community today believes the existing paradigm is inadequate, but they are not sure what should replace it. Because of this uncertainty, practitioners who choose to join the discussion may have an opportunity to reshape leadership preparation programs. 


\section{Interstate School Leaders Licensure Consortium Standards}

The educational environment has been on an evolutionary path throughout this country's history. The redefinition of school leadership is just one more adaptation that is occurring in the educational environment. The current economic conditions on a global scale have drawn much attention to the product of the United States educational system, which is the graduate. Current forces driving education reform are seeded in how the United States maintains economic supremacy in the global context. Urgency for change in how we educate students to be competitive in the global market is the primary force behind the reform process. Murnane and Levy (1996) articulated the value of the new type of skill level needed for students:

Today's schools look much like Ford in 1926. The products they produce-student achievement levels - are not worse than they were 20 years ago; and in most respects, are slightly better. But in those 20 years, the job market has changed radically. Just as the Model T that was good enough in 1921 was not good enough in 1926, the education that was adequate for high-wage employers in 1970 is no longer adequate today. (p. 77) Today's leaders must operate to meet the needs of an ever- changing school environment and to meet the best interest of the children in their schools. Murphy and Shipman (1999) expressed principals of today and the future must:

"Widen the circle of participation in schools, focus on collaboration and shared decision making, and enhance self-efficacy among organizational members. Finally, in facilitating the development of caring community, leaders need to demonstrate the ethic of care to all members of the school community. (p. 216) 
Principals must meet new challenges in today's schools. The need for principals with leadership skills is stronger than ever. Elements for developing professional standards for school leaders were in place according to Murphy \& Shipman, 1999.

The birthplace for the ISLLC standards started with an organization referred to as The Council of Chief State School Officers (CCSSO). The CCSSO is a nonprofit organization comprised of individuals that are heads of departments of elementary and secondary education in the United States. In addition, the District of Columbia, the Department of Defense Education Activity, and five extra state jurisdictions make up the membership of the CCSSO. The CCSSO works by gaining its members' consensus on major educational issues and expresses their opinion to all stakeholders within the educational network. These stake- holders include civic and professional organizations, federal agencies, Congress, and the public. The CCSSO responds to concerns about education issues by using special committees to study and take action on topics of importance in education (Interstate School Leaders Licensure Consortium [ISLLC], 2006)

"The National Policy Board for Educational Administration (NPBEA), under the leadership of its then-corporate secretary, Scott Thomson, created ISLLC in 1994 to develop standards to anchor the profession as it headed into the $21^{\text {st }}$ century" (Murphy, 2005b). The ISLLC initiative was supported by the members of 24 states, a foundational grant from the Pew Charitable Trusts, assistance from the Danforth Foundation, and the National Policy Board for Educational Administration (NPBEA). The member states are Arkansas, California, Connecticut, Delaware, Georgia, Illinois, Indiana, Kansas, Kentucky, Maryland, Massachusetts, Michigan, Mississippi, Missouri, New Jersey, North Carolina, Ohio, Pennsylvania, Rhode Island, South Carolina, Texas, Virginia, Washington, and Wisconsin. In addition, the following 
professional associations are affiliated with the Interstate School Leaders Licensure Consortium (ISLLC):

- American Association of Colleges for Teacher Education,

- American Association of School Administrators,

- Association for Supervision and Curriculum Development,

- Association of Teacher Educators,

- National Association of Elementary School Principals,

- National Association of Secondary School Principals,

- National Association of State Boards of Education,

- National Council of Professors of Educational Administration,

- National Policy Board of Educational Administration,

- National School Boards Association,

- The University Council for Educational Administration.

Starting in 1994 Interstate School Leaders Licensure Consortium (ISLLC) worked on designing model standards for school leaders. Murphy (2005b) noted, "A significant chunk of the standards is supported by the empirical findings from studies of effective schools and from the larger body of research on school improvement in which school effects studies are nested." (p. 159). The objective of the Consortium was to create a set of standards that would provide the basis for reshaping the profession of school administration and to direct action in the academic, policy, and practice (Murphy, 2005b). Using research on productive educational leadership and the wisdom of colleagues, the Consortium drafted standards. The ISLLC standards were developed to set a common core of knowledge, dispositions, and performances to which good leaders would aspire. In recent years, consensus has been building around the 
standards of the Interstate School Leadership Licensure Consortium (ISLLC), which have guided certification reform in many states (National Policy Board for Educational Administration 2002).

The ISLLC standards help principals focus on enhancing educational outcomes. The original purpose in developing the ISLLC standards was to meet the curriculum guidelines for school administration set by the National Council for the Accreditation of Teacher Education (NCATE). The National Council for Accreditation of Teacher Education (NCATE) recently aligned its accreditation standards for leadership-training programs with ISLLC (National Policy Board for Educational Administration, 2002). The ISLLC standards represented a concerted effort to improve the skills of school leaders, which will lead to enhanced faculty involvement and student outcomes (ISLLC, 2006).

"Although numerous sources may be cultivated, norms rooted in the ethos and culture of teaching, as a profession, provide the most effective basis for leadership in a school." (Greenfield, 1995, p. 75). The Consortium believed the framing of the design for building the values would be of great importance in developing good standards. They used a two-pronged approach in the strategic design for leadership standards. The Consortium used research on the linkages between educational leadership and productive school. Each standard begins with the educational leader as the one that promotes the success of each standard (Lashway, 2002). The children's educational outcomes determined which schools would receive the designation of "productive school." Next, the group reviewed significant trends in society and education that hold implications for emerging views of leadership. Our society is becoming more diverse every day. Our society is more ethnically, linguistically, and culturally diverse. At the same time, poverty is increasing while indices of physical, mental, and moral well-being are declining. In addition, the shift to a post- industrial society and a shift to a global marketplace with increasing 
reliance on technology pose new challenges for education. The Consortium held that the standards should address the new type of leadership needed in schools to meet the changing times in our society.

The Interstate School Leaders Licensure Consortium (ISLLC) believed that the standards design would be strengthened considerably if they contained overarching principles to guide the framers' efforts. The overarching principles served two functions during the creation of the standards. First, the principles served as a guide for testing the scope of each standard. Second, the overarching principles helped to give meaning to the standards and indicators during creation. Listed below are the seven principles the Consortium used to guide their work:

1. Standards should reflect the centrality of student learning.

2. Standards should acknowledge the changing role of the school leader.

3. Standards should recognize the collaborative nature of school leadership.

4. Standards should be high, upgrading the quality of the profession.

5. Standards should inform performance-based systems of assessment and evaluation for school leaders.

6. Standards should be integrated and coherent.

7. Standards should be predicated on the concepts of access, opportunity, and empowerment for all members of the school community (ISLLC, 2006).

The work of ISLLC focused on two additional goals when writing the standards. One of the intents for the development of the ISLLC standards was to stimulate dialogue about the quality of leadership across the educational landscape. Many of the stakeholders included state agencies, professional associations, and institutions of higher education, parents, students, and community. The second intent in the creation of the ISLLC standards was to enhance the quality 
of educational leadership within the country's schools. The Interstate School Leaders Licensure Consortium desire was to raise the standards and develop norms for the practice of effective school leadership (ISLLC, 2006).

On November 2, 1996, the Interstate School Leaders Licensure Consortium (ISLLC) adopted standards for school leaders. Approval by the full Consortium was obtained for the adopted standards for school leaders. Neil Shipman, Director ISLLC, and Joseph Murphy, Chair ISLLC (1996) articulated how they wanted the standards used for school leadership practice:

It is the desire of the Consortium to raise the bar for the practice of school leadership. Thus, the standards and indicators reflect the magnitude of both the importance and the responsibility of effective school leaders (ISLLC, 2006, p. iii).

We encourage you to heavily use this document - circulate it widely to members of the public and the profession as well as to the policy-making community. It is through this shared visionof education that school leaders will be successful and that our children will be assured of the education they will need to carry out the responsibilities of the future (ISLLC, 2006, p. iii).

The ISLLC team decided at the beginning of the project to focus on standards. This strategy made sense for a variety of reasons. First, other arenas of educational reform need guide lines or standards to enact reform. The efforts of the Interstate New Teachers Assessment and Support Consortium (INTASC) would be able to use the standards as an appropriate advantage point for reform. In addition, the ISLLC team believed that the standards approach provided the best avenue to allow stakeholders to drive improvement efforts in areas of licensure, program approval and candidate assessment (ISLLC, 2006). 
The ISLLC standards are the first universal set of standards for school leaders. These standards strengthen preparation programs primarily through the manipulation of state controls over areas such as licensure and program approval (Thomson, 1998). Critics contend there is a lack of research to support the standards. The standards rest heavily on the research of productive schools of women and men who lead schools where all children are well educated (Murphy, 2005b).

Creation of the standards for school leaders is a product of the profession. Nine professional associations with close ties to school leadership were included in the process. Professors form school administration programs were involved during the development of the ISLLC standards. The organization of ISLLC only employs an executive director and a halftime secretary with a three-year budget of 250,000 . It would be difficult to classify ISLLC as a bureaucracy due to no physical home, budget, and limitation of employees. The standards are a set of ideas that have spread because of the widespread professional support (Murphy, 2005b). The educational leadership community has endorsed the interstate School Leaders Licensure Consortium's (ISLLC) push for standards (Hess, 2003). The ISLLC standards were formally developed in mid-1994. Approval arrived at the end of 1996. Since that time, the standards have exerted a great deal of influence on the profession of school administration (Murphy, 2005b). The work to find effective school leadership has been a challenge for the past century. The leadership position is ever evolving and a difficult target to obtain. Defining the current era of leadership during an era of transition in society and schooling is a challenging task. Other entities have devoted productive energy to upgrading the profession. All the major professional associations have devoted energy to the issues facing school leadership. The work of ISLLC to create leadership standards is part of a long tradition of continual upgrading in the 
teaching profession. The vision to find quality educational leadership in a changing environment is the challenge of the future.

\section{Connecting Research to Practice}

Schon (1983) described inquiry as a reflective practice in which the teacher is researcher. He described practice as beginning with a knowledge base then becoming an active process involving inquiry, creativity, analysis, and evaluation.

Inquiry is driven by an organization's need to examine its goals and purposes; to decide on programs, curricula, and instructional practices that will advance its ability to realize its examined goals and purposes; and to evaluate whether or not it has been successful in achieving its goals. (Hunkins, Wiseman, \& Williams, 1995).

Inquiry as a reflective practice in leadership is an active process. The principal as the instructional leader must have a strong knowledge base and an understanding of how and why research is needed and when to implement proven teaching strategies. A good practitioner with the understanding of how research works has the building blocks for being an independent leader. For example, Joseph Murphy served as a Public School Administrator for six years (personal communication, March 5, 2009). Dr. Murphy has published over 15 books and 200 articles. In addition, he has worked in conjunction with other members of the education community to create ISLLC leadership standards. Who better to assist in preparing leadership standards than a former leader?

Using a comparison of the health profession field, Portney and Watkins (2000) articulated the value of research being applied to practice:

The ultimate purpose of a profession is to develop a knowledge base that will maximize the effectiveness of practice. To that end, health professionals have recognized the 
necessity for documenting and testing elements of clinical practice through rigorous and objective analysis and scientific inquiry. The concept of evidence-based practice represents the fundamental principle that the provision of quality care will depend on our ability to make choices that are confirmed by sound scientific data, and that our decisions are based on the best evidence currently available. If we look at the foundations of clinical practice, however, we are faced with the reality that often compels practitioners to make intelligent, logical, "best-guess" decisions when scientific evidence is not available. (p. 3)

Research is a systematic method for evaluating the effectiveness of treatments and establishing guidelines to achieve desired outcomes. Whether it is in the school as a teacher or as an administrator leading the school, the desired outcome is growth for all participants in areas of personal, professional, and academics. The ultimate goals are to further the intellectual progress of the profession by contributing to the present scientific base of knowledge through the development of theory and edifying the performance of practitioners. The Professional Development School movement was geared to reflective practices of the classroom teacher and expanded over time to encompass school leaders.

\section{Current Research, School Leadership, and PDS}

Glenn Hubbard (2003) used case studies to establish a profile of efforts to sustain Professional Development Schools. During his research, Hubbard defines a Professional Development School (PDS) and relays the history and culture of the institution.

"Professional Development Schools occur at the intersection of an emergent notion of collaborative work, aimed at the renewal of both the school and the teacher education program, including the professional development of faculty from both domains" (Hubbard, 2003, p. 28). 
The PDS conceptual structure is supported by using the resources of both colleges and schools. It redefines the various relationships among critical stakeholders, which make up teacher education. The PDS term is used to describe various reform movements and strategies for changing the fundamental structure of teacher education programs. A PDS is typically housed at an elementary, middle, or high school. A PDS “is a movement toward a new form of collaboration between higher education and the schools, which links reform agendas in both sectors" (Hubbard, 2003, p. 9).

Historically, the roots of the Professional Development School (PDS) can be traced back to the eighteen hundreds. "It is clear that the movement to develop PDSs embraces many critical, structural and conceptual dimensions of previous efforts to refine and reinvent teacher education, including efforts at "professionalizing" the curriculum in the early part of the century" (Hubbard, 2003, p. 9). John Dewey's laboratory school where pedagogy could be developed through testing, verification, and theory development was linked historically to the PDS concepts.

In a more recent chronological development of the PDS initiative, the Holmes Group recommended the creation of teacher education Professional Development Schools (The Holmes Group, 1990). Almost ten years later, the National Commission on Teaching and America's Future reinforced the recommendations made by the Holmes Group (National Commission on Teaching and America's Future, 1996). The recommendations called for a transformation of teacher learning and a change in how knowledge is constructed in a teacher-learning context. In addition, the establishment of a PDS implies that research is conducted in a collaborative process by both school and university or college faculty. This notion of collaboration is sometimes referred to as simultaneous renewal (Goodlad et al, 1990). Fullan reminds us that the PDS 
initiative is an enormous complex set of ideas; "if they are to work... the culture of the school and the culture of the university change and begin to overlap in organic ways" (Fullan, 1991, p. 323).

An event of historical importance with the PDS evolvement occurred in 2001. The American Council on Education (AASCU) sponsored a Task Force to examine the Professional Development for Teachers. The focus of the task force was to identify general reforms in teacher education by focusing on the responsibilities of universities and colleges. The report grounds its recommendations on three assumptions about universities and teacher education.

1. The improvement of teacher quality is a systemic issue, involving the interaction of public policy and many groups and agencies

2. The president and provost must be strong visible leaders for teacher education

3. Within universities, teacher education is an all-university responsibility (Lewis, 2001, p. 13).

This report cites the importance of professional development programs for the needs of schools and communities. The Professional Development School structure is one of the strategies the report recommends to achieve higher quality programs for pre-professional teachers and university professionals. The report lists recommended reforms needed to sustain PDS relationships. The report suggests college presidents should evaluate and find ways for promotion and tenure policies to be integrated in formal evaluations for higher education faculty. In addition, the report suggests universities should develop incentives for faculty participation in collaborative activities and action research in school-college collaborations (Hubbard, 2003).

During 2001, the National Council for Accreditation of Teacher Education (NCATE) created its standards for Professional Development Schools. The setting of norms by NCATE 
was an important step in ensuring quality for the PDS movement. The establishment of the standards brings "rigor to the concept of PDSs, so that its potential will not be lost" (National Council for the Accreditation of Teacher Education, 2002, p. 2). The PDS Standards developed by NCATE were intended to create a developmental framework, with detailed descriptive rubrics. The guidelines from the rubrics would guide institutions in assessing their own PDS work and create a set of norms for a PDS.

The culture of the PDS initiative must marry the various members of the educational community allowing them to work as a cohesive unit. The success of PDSs "has rested upon the development of participants as joint, self-empowered problem-solvers, framing their own unique PDS questions and wrestling with their own unique circumstances to develop locally appropriate strategies for change" (Robinson \& Darling-Hammond, 1994, p. 204). Teitel writes that "for any effect of involvement with PDSs to be observable, first there must be evidence that distinct approaches, techniques, or philosophies have evolved which distinguish PDS-related teacher preparation activities from those in the mainstream of the university" (Teitel, 1992, p. 77). Teitel believed significant structural and ideological change must become an integral part of the institutional culture for the PDS initiative to be successful. For the PDS culture to be complete and to be an effective practice, teacher education preparation programs must be tied closely with school partners in defining parameters of best practices in teaching, administration, and research (1992).

"It is clear that in any broad-based cross-institutional involvement with external partners, a significant number of artifacts of the organizational culture for teacher education programs must change" (Hubbard, 2003). To accommodate the development of Professional Development 
Schools, concepts and structural key features of organizational practice must change. Hubbard (2003) articulated the importance of organizational change:

For faculty and staff, these shifts may include differences in workload determination, hiring practices, and the reward structure, including both promotion and tenure criteria; for administrators, new forms of leadership must be promulgated, based in commitment to the innovations from within the teacher education program, as well as buttressed without from the institution at large. (p. 19)

Maintaining the balance between past cultures in higher education and public schools will be a challenging task. Fullan (1991) observed: "If it is hard to get two people to cooperate, imagine trying to get huge institutions like governments, universities, districts, schools, and unions to work together on complex matters" (p. 290). The challenge of PDSs for teacher preparation units and public school partners is the need to define how the process of conceptual renewal will occur and be maintained (Gabbard, 2003). School leadership is the tie that maintains the ongoing concept of renewal.

\section{Dissertation Focused on Administrators' Perceptions of a Change Initiative}

The National Commission on Excellence in Education (1983) recommended reform in the area of vocational education. The imperative for educational leaders today is to prepare young people emerging from high school for college or give them the workforce skills needed for today. The knowledge base continues to expand making the lack of skills more pronounced than ever before. Businesspersons like Bill Gates were suggesting students were not prepared for postsecondary education or the world of work. Leaders of tech-prep have the task of changing the learning process to a more sophisticated knowledge of technology. 
Salaiz (2004) used a case study to view the perceptions of administrators during a time of change to the institution's vision. The Tech Prep reform initiative created serious challenges for the administrators. During this research, the key to any new initiative according to Salaiz is the involvement of key administrators and the principal. Common barriers encountered during the new initiative included lack of time, inadequate facilities, insufficient funding, communication gaps, and staff mobility.

Lyndon B. Johnson held that all problems can be solved working together, but few problems can be solved by ourselves. School administrators face the issue of solving many problems, and they need individuals working collaboratively with them. School principals are often the people most likely to put together the various stakeholder groups for reform. It is critical in a PDS system for site based coordinators to be the lynch pin in organizational operations.

\section{Dissertation Focused on School Based Site Coordinators view of a PDS}

The view of relationships within a PDS through a Site Coordinator's eyes holds valuable information. Kess' (2002) study looked at the perceptions of teachers as reported by schoolbased site coordinators in PDSs. The study design used qualitative methods through interviews of site coordinators and the review of documents concerning events, policies, and procedures. This study clarified the substantive contributions the PDS experiences have on the expanding professional roles in areas of leadership, teacher professionalism, center of pedagogy, and clarifying the PDS mission.

A site coordinator fills many roles on a daily basis. Many of these roles include being a juggler, negotiator, or matchmaker, raising the PDS flag, unifier, and change agent. The juggler is responsible for coordinating various PDS activities. For example, the site coordinator avoids 
scheduling some type of professional program during an education class. As the negotiator or matchmaker, many responsibilities are shared with university and partnered school faculty. It is the matchmaker's responsibility to coordinate the placement of teacher candidates in varied field placements. There will be times during the placement process that personalities may influence placements. Raising the PDS flag involves the building of public relations. The coordinator needs to make more teachers aware of what the PDS is about and try to gain additional support. Campus friendly for the PDS means additional opportunities for research and field placements. The Unifier finds ways to increase the communication between the school-based coordinator and the faculty for sharing information on current PDS initiatives. Finally, the change agent facilitates changes through action research. With all participants working with the same information and vision, the PDS has a better chance of being successful Kess (2002.

Teachers within a PDS have opportunities to assume greater responsibilities in expanding their professional role. Teachers, who assume roles extending beyond the classroom, exercise skills in areas of leadership among their colleagues. These expanded leadership opportunities for individuals allow them to share responsibilities in a variety of areas and positions. The culture created by the PDS in the partnership with the public school and university provides additional opportunities to experience growth in leadership positions. For example, liaisons, site steering committees, and positions serving on PDS councils involve the use of leadership skills. The school-based site coordinator position exists in a PDS partnership; teachers who assume this role have the potential to enhance their knowledge and hone leadership skills. One issue that has been a down side with the added responsibilities is one individual wearing two hats. Instead of giving full attention to one job, an individual must split their focus and time with two positions. 
"The constraints of having a full-time job as a teacher and their PDS role was a common problem expressed by several school-base site coordinators" Kess (2002, p. 178).

Kess defined professionalism as a teacher going beyond the classroom responsibilities. The teacher participates in practices or relationships such as; leadership responsibilities, continuing professional development and enhancement of content knowledge or pedagogical skills to expand their service to the profession. The PDS structure promotes inquiry for all stakeholders within the system.

Undergirding our reform efforts is the commitment to inquiry in schools as the basis for professional practice of both university and school faculty, and to the idea that reflection and inquiry are essential to the renewal of schools and the teaching profession...we believe strongly that teachers who are reflective in their practice will collectively have a stronger voice in changing their individual classrooms and the schools in which they work (Norlander, Case, Reagan, Campbell \& Strauch, 1997, p. 44).

The underlying theory for enhancing teacher professionalism is to improve teacher education and teaching practices (Richardson Foundation, 1993).

Kess (2002) listed the factors supporting and hindering the professionalism of a teacher serving as site coordinator. The factors identified as hindering the professionalism of the teacher as school-based site coordinator are:

(1) the financial constraints,

(2) the lack of time,

(3) changes at the university level,

(4) changes at the district bureaucratic level.

The factors identified by Kess as supporting the professionalism of school-based 
site coordinator were:

(1) the university's response to bridging school and university cultures;

(2) the supportive nature of the university facilitator;

(3) the school administration; and

(4) the school faculty. (p. 68)

Kess (2002) believed the PDS represented the center of pedagogy. Pedagogy is both a concept and setting when associated with a PDS. The PDS should not exist as an entity to itself. The central idea is to join all participating entities under one roof or purpose. Schools, School Districts, Schools of Education, and Departments of Arts and Sciences need to make a commitment to the PDS for it to be successful. Kess (2002) articulated the value of a reflective practitioner:

Findings emerging in this study supported the PDS literature concerning teachers in this role who believed they have benefited in their own learning and teaching practices because they have become practitioners that are more reflective. In effect, as designated exemplary sites and subjects of inquiry-based research, this PDS role encouraged reflection and self-analysis to offer opportunities for the teachers as school-based site coordinators to align their professional development and pedagogical practices with theory. (p. 266)

Goodland (2000) maintains the PDS has a unique mission to support both individuals and institutions during the transformation process. The evolving nature of a PDS allows teachers to experience being leaders and learners. In turn, these individuals can take newly acquired skills and lead institutions toward a proactive agenda for education. Ultimately, the PDSs may boost public confidence that is needed to support the teaching profession. This support allows more 
school wide research opportunities, additional instructional activities for pre-service teachers, and additional leadership opportunities for teachers (Kess, 2002).

\section{Use of Research Based Leadership Practices by PDS Principals}

Susan Stephenson Field (2008) studied highly effective PDS principals through interviews to determine how they used research-based leadership practices to lead their partnership schools. School principals are critical to school success and have a great impact on student achievement (Marzano, McNulty, \& Waters, 2005). Past research has consistently found positive relationships between principal behavior and student academic achievement (Cotton, 2003). "The principals in your PDS need to build community, nurture leadership in a variety of areas, and support and negotiate the change process (Teitel, 2003, p. 70). The NCATE standards are closely aligned to the current leadership research on principal tasks and practices that are connected with student achievement (Field, 2008). Principals need to know where their efforts will have the biggest payoff and proceed accordingly (Leithwood, 2004).

The principal serving as the educational leader must direct the efforts of resources to maximize returns. The principal must have a clear mission and vision in place for the school. In addition, the principal must be willing to be share leadership, invest in professional development, and foster a learning community to achieve the full potential of the organization.

A sense of mission is necessary for all organizations, but especially important for schools. A school may have a clear purpose and mission, but not all stakeholders may have the same vision. Without a clear understanding of the mission, the stakeholders' lacks direction and achievement will suffer (Gullatt \& Lofton, 1996).

A shared vision is essential for high performing schools. Marzano et al. (2003) described shared vision as: 
...focus that refers to the extent to which the leader establishes clears goals and keeps those goals in the forefront of the school's attention. This is done effectively when the principal safeguards against expending huge amounts of energy and resources on school improvement initiatives that go nowhere. (p. 50)

Principals must reach out to stakeholder groups for the shaping and support of the school vision. The principal must engage teachers, parents, students, and others to share in creating the vision. The purpose of the process for the sharing and shaping the vision together is aimed at helping all stakeholders understand the organization and its activities and goals (Hallinger \& Heck, 2002). It is the principal's responsibility to ensure the vision is widely known and connect to the goals for support throughout the school community. The school needs to have a clear academic mission and the mission must be communicated to the staff (Hallinger, 2003).

Principals that share the decision-making process tend to be successful. They ensure everyone is involved by having the needed information and training in place to make the process productive (Cotton, 2003). Marzano et al. (2003) described shared leadership in allowing others the opportunity for input dealing with the implementation of important decisions and policies. Leithwood et al. (2004) felt that the empowering of others to help in making significant decisions was the key for leaders in giving greater voice to the community of stakeholders. It is helpful for some leadership functions to be performed at different levels of the organization. This type of responsibility stimulates the thinking of people about the decision making process throughout the organization. A growing volume of research finds that when principals empower their professional staff through shared leadership, everyone benefits including students (Cotton, 2003). 
A heavy investment in professional development for teachers and principals in the fundamentals of classroom instruction is critical for the success of a school (Elmore, 2000). Principals from high-achieving schools offer more professional development activities than lower achieving schools. High achieving schools offer more varied types of professional development (Cotton, 2003). Leaders determine the direction of the professional development agenda. Reeves (2006) recommended that schools center their attention on specific things: What to teach, how to teach it, how to meet the needs of individual students, and how to build internal capacity. With an emphasis on internal capacity, the leadership of professional development efforts comes from the faculty itself, and a large part of professional education takes place in the classroom in the context of authentic teaching. (p. 86)

The principal should be interested in fostering a learning community. Central to the notion of a learning community is the concept of inquiry as part of professional development (Teitel, 2003). A learning community involves colleagues talking about the act of teaching, observation of teachings, and reflecting on individual instruction. Silins, Mulford, \& Zarins (2002) believe organizational learning is more likely to occur where sufficient resources and time to develop professionally are available and where staves are looking for opportunities to increase knowledge and skills. In the end teachers feel they own the program, and this leads to a comfort level where individuals are willing to take professional risks. This kind of climate is necessary for the active place of innovative programs (Field, 2008).

Bolman and Deal (2003) articulated the powerful tool of reframing for leaders. The use of the reframing technique allows principals to look at a situation from more than one angle. In order to build this climate of self- renewal a principal must have a working knowledge of the 
way they operate. Leaders must know from which viewpoint the stakeholders of the schoolwork. An understanding of both viewpoints and frames will give principals an opportunity to share their vision. The stake holders must receive the message of renewal in a context or frame that they understand.

\section{Dissertation Focused on the Essence of the Principal's Role in a PDS}

Tilford (2007) studied the role of a principal in a PDS. The Boleman and Deal concept of Four Frames was used to analyze the leadership styles. "The study indicated that Professional Development School work can serve the professional growth needs of principals in addition to facilitating growth of staff and students." (p. 16). The principal is the central hub of connection for all areas of the PDS. "Given the importance of the principal in the success of the PDS, we need to better understand the principal's role in PDS leadership.” (p. 18). Also noted in Tilford's study were limited research on principals and their role as leaders within the PDS setting.

Tilford alluded to the PDS movement as being a significant reform movement in the United States over the past decade. Teitel (1999) supported the thought of the PDS being influential over the past decade. Even with the PDS being an agent of reform little attention has been given to the leadership of the PDS. According to Trachtman and Levine (1997), "To date, little attention has been paid to the kind of leaders needed to build Professional Development Schools, or to the nature of effective leadership for collaborative, restructuring environments. Indeed, frequently leaders are not even identified." (p. 76). With the limited amount of attention or research on the topic of PDS leadership, Tilford selected to study the role of PDS principals working in a collaborative system. The study reviews the literature concerning the 
history of the PDS movement, the NCATE Standards for Professional Development Schools, and characteristics of a PDS principal.

Tilford pointed out the progressive ideas the National Commission on Teaching and America's Future (NCTAF) (1996) supported. Reforming education with PDSs was an idea NCTAF supported with the proposal of yearlong internships for pre-service teachers in PDSs. The following comments demonstrate the support NCTAF wished-for with PDSs.

In tandem with these new program initiatives, more than 200 (out of 1,200) schools of education have created 'Professional Development Schools' that, like teaching hospitals in medicine, provide new recruits with sites for intensively supervised internships where they can experience state-of-the-art practice that is aligned to their coursework. They also provide sites for research and school-and university-based faculty, creating more powerful knowledge for teaching by putting research into practice and practice into research. (p. 80)

At the same time, Levine (1992) was calling for the movement toward a collaborative system to continue. Levine referred to this type of system as a professional practice school. She wanted the professional practice school to operate with dual responsibilities. First, the professional practice school would be responsible for educating pre-service teachers and supporting ongoing research with the purpose of improving practice. Levine believed the academic and social learning of the children was the top priority. However, Levine stressed the importance of equity, inquiry, and building a nurturing climate of learning for all participants. As Levine stated, "If one hopes to have teachers teach in different ways, one must change the way teacher are taught themselves" (p. 13). "Clearly, a strong connection exists between Levine's call for professional practice schools and the Professional Development Schools 
envisioned by the Holmes Group as they both emphasized creating a learning culture for all participants" (Tilford, 2007, p. 26).

Different terms have alluded to or been associated with and even described what a Professional Development School is. Abdal-Haqq (1998) noted in various literature the different terminology associated with PDSs. Many of the terms found in the literature included clinical schools, partnered schools, and professional practice schools. Abdal-Haqq (1998) identified the goals and principals that were common to those schools regardless of their name:

Preparation of pre-service teachers and other school-based educators; b) professional development of practicing teachers and other school-based educators; c) exemplary practice designed to maximize student achievement; and d) sustained, applied inquiry designed to improve students and educator development. (p. 6)

Teitel (1998) contributed to the national conversation with his thoughts related to School University collaboration and the connection to PDSs. In his writings, he defined a Professional Development School as "complex partnerships formed by two or more institutions in mutual renewal and simultaneously trying to expand professional development opportunities at both institutions, engage in research and development, and improve the education of children, adolescents, and prospective teachers." (p. 1)

As the conversation about PDSs continued among stake- holders in education, The National Council for Accreditation of Teacher Education (NCATE) responded. The group NCATE created a set of standards outlining a series of norms for work associated with organizations having different names but the same purpose as a PDS.

The National Council for Accreditation of Teacher Education published a report in spring of 2001 titled Standards for Professional Development Schools. The report was released after 
NCATE had completed three years of piloting the standards. Five standards addressing the characteristics of PDSs were outline by NCATE. The PDS standards are connected and overlapping. Consequently, the standards should be viewed holistically. Each PDS standard consists of several elements.

Standard I (NCATE, 2001) focused on the concept of learning communities. Five elements were identified within standard I:

(1) supports multiple learners,

(2) work and practice are inquiry-based and focused on learning,

(3) a shared vision of teaching and learning grounded in research and practitioner knowledge, (4) serves as instrument of change,

(5) creates an extended learning community.

The element of supporting multiple learners is addressed in the environment of the PDS. The learning structure within a PDS climate involves P-12 students, candidates, faculty, and other professionals. An example of multiple learners being supported would occur during the field experience. P-12 students, pre-service teachers, and faculty members participate in positions such as mentors, co-teachers, and colleagues in study groups. Professional development opportunities are offered to all through seminars, committees, and other professional activities.

Element Two is addressed in the PDS through inquiry-based practice weaved together in learning, accountability, and faculty development. Inquiry is used in individual classrooms searching for the best approaches for teaching and learning.

A shared vision of teaching and learning grounded in research and practitioner knowledge is the third element. The PDS partners believe that children and adults learn best by 
practice in context. The learning experiences in the PDS reflect the most current research and advice from seasoned practitioners.

Serving as an instrument of change is the focus of element four. The PDS serves as a tool for education reform and school improvement. The development of new approaches and examining teaching practices fits into the PDS culture.

Element Five focuses on the extended learning community. Multiple partner institutions and established relationships is the combination that leads to an extended learning community. The PDS structure is the place to understand and address professional and cultural differences of various stakeholders.

Barth (1990) feels it is the principal's responsibility to ensure the learning community is in place. The principal must keep the communication lines open to individuals both inside and outside the school to keep relationships relevant.

Standard II deals with accountability and quality assurance. Five elements were identified within Standard II, and they are: (1) developing professional accountability; (2) assuring public accountability; (3) setting PDS participation criteria; (4) developing assessments, collecting information, using results; and (5) engaging within the PDS context (NCATE, 2001).

Accountability and quality assurance is connected to the PDS's purposes and mission. A continuous process of assessment is completed on the local, state, and national level to insure accountability. The results from the prior assessments are used to make constructive changes at the partnership levels. Public accountability in element two is handled by keeping the public aware of the results of the PDS work. 
Element Three participation criteria are satisfied by the accreditation process. The public needs to be aware of the open recruitment of PDS faculty. PDS school faculty is to be licensed in the fields they teach and supervise.

The creation of standards for pre-service teachers matches with one part of element four. In addition, PDSs revise their teaching and learning approaches after the completion of assessments. This is measured through the assessment of P-12 student achievement. The idea is to collect information and use it to improve performance. This concept is strongly connected with the concept of renewal. The final element of engaging the partnership school in PDS context is the key to increasing the stakeholder's knowledge about their school. This effort will allow PDS personnel to be current with national, state, and local policies that effect the operation of their school.

The school's goals and mission are strongly connected to the PDS accountability and quality assurance. The principal's role is to establish the individual school goals within the federal, state, and local mandates (Matthews \& Crow, 2003). "The principal also determines the criteria for success in the PDS program at the school” (Tilford, 2007, p. 29).

Standard III (NCATE, 2001) focused on collaboration. Three elements were identified within the collaboration standard; (1) engage in joint work; (2) design roles and structures to enhance collaboration and develop parity; (3) systematically recognize and celebrate joint work and contribution of each partner.

Elements One and Two consist of joint work and roles in a collaborative effort. Both of these are fully integrated into the decision making process of a PDS. The objective is to strive for equal participation while limiting the power of an individual or partner within the collaborative. For example, the PDS partnership committee includes representatives from all 
stakeholders. The final element of celebration has been integrated into the school's culture. Tilford (2007) articulated the role of the principal collaboration standard.

The principal is most often the person responsible for deciding the structures that exist within his school. A principal lays the groundwork for collaboration by inviting all staff to participate. Principals can also design structures that make participation easy for staff members. Celebrating collaboration through sharing inquiry projects at faculty meeting or encouraging events where all participants are invited help build the collaborative spirit. (p. 30)

Standard IV (NCATE, 2001) focused on diversity and equity. Three elements were identified within the collaboration standard; (1) ensure equitable opportunities to learn; (2) evaluate policies and practices to support equitable learning outcomes; (3) recruit and support diverse participants.

Elements One and Two are addressed by the PDS nurturing climate for learning. Opportunities for learning will occur for all students including those from diverse ethnic and socio-economic backgrounds. The assessment procedures will ensure that equitable outcomes apply to all students. To support a diverse culture of learning the PDS should include diverse participants from the school and university. This type of inclusion will address the third element of recruitment of diverse participants. "As principals do the hiring for schools, they are the key to bringing in diverse participants. The structures and policies a principal designs can foster equitable opportunities for learning" (Tilford, 2007, p. 30).

Standard V (NCATE, 2001) focuses on structures, roles, and resources. Five elements were identified within Standard V and are; (1) establish governance and support structures; (2) 
ensure progress towards goals; (3) create PDS roles; (4) resources; (use effective communication.

Element One is the establishment of governance and support structures. The PDS uses the governing council to engage the faculty and to provide oversight. Many of the rules of oversight are directly tied to the support of the PDS, as the principal deems necessary. Making public the goals of the PDS, helps to move the PDS initiative forward. This type of effort addresses standard two. Elements 3 and 4 are addressed when the modification of positions and the reallocation of resources occur to strengthen the PDS. All of these changes will be served with the effective use of communication as stated in standard five. "PDS partner institutions create communication links with the broader school district and university communities. Partner institutions receive and exchange information about PDS work plans, resources, and structures (NCATE, 2001, p. 16).

Tilford (2007) summed up the role of the principal when working with NCATE standards.

The NCATE (2001) Standards for PDSs, although not written specifically for the principal, provide a compass whereby principals need to guide their staff and other PDS participants. As indicated, many of the standards outlined could not be accomplished without the effective leadership of a principal. (p. 31)

The characteristic of the PDS principal play a key role in the development of the NCATE standards. Kersh and Mastal (1998) analyze the role of the principal.

The school principal is a key person in collaborative school reform. The principal's position in these partnerships is uncomfortable: he or she must accommodate the administrative expectation of the principal as a strong leader while simultaneously releasing "power" to 
teachers to participate in decision-making in the collaborative.... For any sustained partnership, the principal must continuously, vigorously, and openly support the partnership (p. 6).

Tilford held understanding the characteristics of an effective PDS principal were imperative due to the importance of the leadership position to the PDS movement. Tilford used the information from Foster, Loving, and Shumate (2000) that the essential function of the principal must be involved in all stages of the PDS work to support the importance of understanding the principal position. In addition, Cramer and Johnston (2000) pointed out that many articles emphasize the importance of the principal in school-university collaborative experience, yet a limited number of studies have focused on the principal's role separate from teachers, students, and university personnel.

\section{Summary of Related Literature}

There is a shortage of qualified principals today (Murphy, 2005). The shortage of qualified school leaders is an issue of extreme importance today. The present climate in school leadership finds current leaders leaving and the new pool of leadership applicants being described as shallow (Keller, 2000). In addition, many of the qualified individuals are unwilling to assume the responsibilities of the principal. There are many opinions as to why the condition of leadership is in such difficulty. Opinions and beliefs vary as to what the real issue is for administrators. Some judge that principals are over-burdened, while others blame the failure of leadership preparation programs. These critics believe that the programs have failed to plan for the next generation of school leaders. The specific reason for the shortage is still unknown. However, the importance of the principal position is without question. The principal is the key 
player in the direction a school system will follow (Hammonds, 2002). Other individuals referred to the principal as the gatekeeper of change (Fullan, 2004).

The professionals who work in Professional Development Schools (PDS) are expected to engage in the process of personal and professional reflection to ensure professional growth. It is the PDS culture that evolves from all those working in this process of personal and professional reflection that enhances the ability of the organization and individuals to maintain a consistent state of renewal. These areas of renewal involve relationships among a list of stakeholders such as, teachers, pupils, parents, and principals (Murphy, 2005). Central to any PDS is the professional development of educators and administrators (Teitel, 2003).

The authors of the (ISLLC) standards intended to provide a picture of quality educational leadership for all stakeholders. The ISLLC standards present a common core of knowledge, dispositions, and performances that will link leadership to productive schools and enhance educational outcomes (Murphy, Shipman, 1999).

Trachtman and Levine (1997) addressed the non-awareness of what the principal of today and the future looks like.

To date, little attention has been paid to the kind of leaders needed to build Professional Development Schools, or to the nature of effective leadership for collaborative, restructuring environments. Indeed, frequently leaders are not even identified. Most literature about Professional Development Schools focuses on the changing role of teachers in school-university collaboration or on the characteristics of the new school culture. In fact, PDSs are typically thought of as teacher-centered projects. (p. 76) This study will take a precise look at how principals perceive their work in two different leadership settings, Professional Development Schools (PDS) versus non-Professional 
Development Schools. Through this type of study on educational leadership, a better understanding about good leadership in accordance with the ISLLC standards will be gained as perceived by principals. The information derived from the study on PDS and non-PDS principals will help to gage whether PDS principals and non-PDS principals understand and model good leadership outlined by the ISLLC standards.

The purpose of this study is to develop an instrument that focuses on the perceptions of PDS principals in alignment with the Interstate School Leaders Licensure Consortium (ISLLC) standards. This study will add professional knowledge to the base of information already present concerning the PDS culture. Principal perceptions from this study will gauge what a PDS culture looks like from their perspective. During the course of this study, an instrument will be constructed using the perceptions of leaders in alignment with ISLLC standards to take a snapshot of how supportive the PDS climate is for principals. In chapter 3, the research methodology and research design are described. 


\section{Chapter Three: Research Design}

Individuals have been concerned with the management of the human organism. The human component is complex, and the most important aspect of any organization. Rensis Likert (1967) articulated the importance of managing the human component.

All the activities of any enterprise are initiated and determined by the persons who make up that institution, plants, offices, computers, automated equipment, and all else that a modern firm uses are unproductive except for human effort and direction. Human beings design or order the equipment; they decide where and how to use computers; they modernize or fail to modernize the technology employed; they secure the capital needed and decide on the accounting and fiscal procedures to be used. Every aspect of a firm's activities is determined by the competence, motivation, and general effectiveness of its human organization. Of all the tasks of management, managing the human component is the central and most important task, because all else depends upon how well it is done. (p. 1)

Research on the management of the human resources can be derived from verifiable information resulting from rigorous, quantitative research. Studies can be repeated to test the validity of the findings. Quantitative research anywhere in the world can add to knowledge already in place concerning the management or leadership of human recourses (Likert, 1967).

The research problem at the center of this study is how to begin the process of measuring principals' perceptions in alignment with what has been established as general principles of good school leadership. The notion of professional development schools as a work concept for educators was established by Dewey in the early nineteen hundreds. Instrumentation that would indicate a principal's perception on holistic professional growth would be invaluable. An 
instrument must be developed to gather data to answer the following questions presented in this study. Is there a relationship between involvement with a professional development school and leaders' perceptions of their work, corresponding to the ISLLC standards? Additionally, will PDS principals have higher scores as opposed to non-PDS principals on an instrument designed to test perceptions in alignment with the ISLLC standards?

The survey instrument developed to measure principal perceptions has six domains with 31 items. The items are correlated to the dimensions of the Interstate School Leaders Licensure Consortium (ISLLC) national standards. For the purposes of this study, the following six areas have been labeled in the survey:

Domain 1: Visioning

Domain 2: Advocating

Domain 3: Management

Domain 4: Collaboration

Domain 5: Ethics

Domain 6: Community Relations

\section{Research Questions and Hypotheses}

Is there a relationship between involvement with a Professional Development School and how leaders perceive their work in accordance with the ISLLC standards? The research hypotheses for this study relate to each of the Interstate School Leaders Licensure Consortium standards (ISLLC) covering the following six dimensions:

The Domain, null hypothesis, and alternative hypothesis for each research question follow. 
Domain 1 (Visioning): An educational leader promotes the success of every student by facilitating the development, articulation, implementation, and stewardship of a vision of learning that is shared and supported by all stakeholders.

$\mathrm{H}_{0}$ : There is no statistically significant difference in Domain $\underline{1}$ scores when comparing individuals who are principals of Professional Development Schools with those who are principals of non-Professional Development Schools.

$\mathrm{H}_{1}$ : There is a statistically significant difference in Domain $\underline{1}$ scores when comparing individuals who are principals of Professional Development Schools with those who are principals of non-Professional Development Schools.

Domain 2 (Advocating): An educational leader promotes the success of every student by advocating, nurturing, and sustaining a school culture and instructional program conducive to student learning and staff professional growth.

$\mathrm{H}_{0}$ : There is no statistically significant difference in Domain $\underline{2}$ scores when comparing individuals who are principals of Professional Development Schools with those who are principals of non-Professional Development Schools.

$\mathrm{H}_{1}$ : There is a statistically significant difference in Domain $\underline{2}$ scores when comparing individuals who are principals of Professional Development Schools with those who are principals of non-Professional Development Schools.

Domain 3 (Management): An educational leader promotes the success of every student by ensuring management of the organization, operations, and resources for a safe, efficient, and effective learning environment. 
$\mathrm{H}_{0}$ : There is no statistically significant difference in Domain $\underline{3}$ scores when comparing individuals who are principals of Professional Development Schools with those who are principals of non-Professional Development Schools.

$\mathrm{H}_{1}$ : There is a statistically significant difference in Domain $\underline{3}$ scores when comparing individuals who are principals of Professional Development Schools with those who are principals of non-Professional Development Schools.

Domain 4 (Collaboration): An educational leader promotes the success of every student by collaborating with families and community members, responding to diverse community interests and needs, and mobilizing community resources.

$\mathrm{H}_{0}$ : There is no statistically significant difference in Domain $\underline{4}$ scores when comparing individuals who are principals of Professional Development Schools with those who are principals of non-Professional Development Schools.

$\mathrm{H}_{1}$ : There is a statistically significant difference in Domain $\underline{4}$ scores when comparing individuals who are principals of Professional Development Schools with those who are principals of non-Professional Development Schools.

Domain 5 (Ethics): An educational leader promotes the success of every student by acting with integrity, fairness, and in an ethical manner.

$\mathrm{H}_{0}$ : There is no statistically significant difference in Domain $\underline{5}$ scores when comparing individuals who are principals of Professional Development Schools with those who are principals of non-Professional Development Schools.

$\mathrm{H}_{1}$ : There is a statistically significant difference in Domain $\underline{5}$ scores when comparing individuals who are principals of Professional Development Schools with those who are principals of non-Professional Development Schools. 
Domain 6 (Community Relations): An educational leader promotes the success of every student by understanding the profile of the community, responding to, and influencing the larger political, social, economic, legal, and cultural context.

$\mathrm{H}_{0}$ : There is no statistically significant difference in Domain $\underline{6}$ scores when comparing individuals who are principals of Professional Development Schools with those who are principals of non-Professional Development Schools.

$\mathrm{H}_{1}$ : There is a statistically significant difference in Domain $\underline{6}$ scores when comparing individuals who are principals of Professional Development Schools with those who are principals of non-Professional Development Schools.

The null hypothesis is the core idea in hypothesis testing. The null hypothesis is what the researcher usually hopes to reject, thereby substantiating its opposite. The concept is comparable to the legal assumption that a person is innocent until proven guilty. It suggests no relationship exists between variables until reasonable evidence is presented to convince us otherwise (Portney \& Watkins, 2000).

The rejection or acceptance of a null hypothesis is based on some level of significance (alpha level) as a criterion. The alpha level is the point of chance a researcher is willing to take of committing an alpha error or type I error. The alpha percentage is the probability that a type I error has been committed. "Thus, an alpha level of .01 is a more difficult criterion to satisfy than a level of .05" (Vogt, 2005, p. 7). For example, a .01 alpha or 1\% level indicated that a difference in means that was the highest level tolerable between the experimental and control groups would have resulted from an error in less than 1 out of 100 replications of the experiment. If the alpha was .05 or $5 \%$, the indication is a difference in means as large as that found between the experimental and control groups and would have resulted from a sampling error in less than 5 
out of 100 replications of the experiments. This suggests a $95 \%$ probability that the difference was due to the experimental treatment rather than to sampling error. Upon review of the prior information, the alpha for this study will be .05 or $5 \%$.

Participants. The principals selected for this study will come from a convenience sample from public county schools within West Virginia. All principals who have an accessible public e-mail address will be surveyed within West Virginia. A limitation to the sampling procedure will be connected with principals' participation. One issue will be the availability of e-mails for principals, and the other issue will be convincing principals of the importance of completing the survey. A letter requesting support for this survey will be sought from state leaders. One advantage of a relationship study is that all the data may be collected within a relatively short period. The survey instrument may be administered in one session by a mass email (Gay, Mills, \& Airasian, 2006).

The population of this proposed study is the group of principals with e-mail addresses from schools in West Virginia. The Control Group will be the principals from non-Professional Development Schools and will be self-selected by their individual response of non-PDS or PDS on the survey. The Experimental Group will be the principals from professional development schools and will be self-selected by their individual response of PDS or non-PDS on the survey. The proposed survey will look at the results of the data in the aggregate. An aggregate is "a group of persons, or other units of analysis, that have certain traits or characteristics in common without necessarily having any direct social connection with one another, such as the population of a city. Also called aggregation" (Vogt, 2005, p. 5). The groups of individuals being studied are principals within the state of West Virginia with e-mail addresses. The characteristics dividing the principals will be the connection with Professional Development Schools. 
Instrument characteristics. This instrument will compare the perceptions of principals working within Professional Development Schools to the perceptions of non-PDS principals by utilizing the national ISLLC standards. On November 2, 1996, the Interstate School Leaders Licensure Consortium (ISLLC) adopted standards for school leaders. The ISLLC standards represented a concerted effort to improve the skills of school leaders. The goal of the ISLLC standards is to enhance faculty involvement and improved student outcomes (ISLLC, 2006).

The descriptions of the dimensions of the six domains served as the framework for the development of the survey instrument. The purpose of the survey was to determine the perceptions of principals working within a PDS structure versus a non-PDS setting in alignment with the ISLLC standards

Survey instrument development. "Data collection is the soul of a study. The validity or 'truth' of all research depends on accurate data" (Fink, 2005, p. 106). One of the primary responsibilities during the review of literature is to evaluate the value of the study. The core of the literature is built on the data. The data must be unyielding in order for the study to be sound.

Question writing for surveys is a challenging art. Many things are competing for attention in the question writing process. Failure to address certain issues leaves the door open for a catastrophe in a self-administered survey. Dillman (2007) expressed the difficulty in writing a survey question:

The goal of writing a survey question for self-administration is to develop a query that every potential respondent will interpret in the same way, be able to respond to accurately, and be willing to answer. However, in practice, producing good questions is often difficult (Dillman, 2007, p. 32). 
Step one in the development of a survey was to define the intent of the instrument. In discussions, my committee chair brought to my attention the Interstate School Leaders Licensure Consortium (ISLLC) standards for leaders. With the ISLLC standards in place, the connection between principals' perceptions became the focus of the survey. The variable separating the principals will be their involvement with Professional Development School as leaders. The decision was made to base the instrument on the ISLLC standards.

Next, dialog was opened with some of my committee members, fellow cohorts, and experts. Literature was reviewed on the ISLLC standards and the development of survey instruments. After the prior efforts, each standard in the ISLLC document was broken into a domain. Each domain was narrowed to a specific purpose. For example, the ISLLC Standard One was now reflected in the survey by Domain 1 titled, Visioning. Under each domain, the key points were taken and used as survey items.

The first draft of the instrument involved the use of a Likert-type scale. Vogt (2005) stated, "Likert scales, and Likert-like scales, are the most widely used attitude scale types in the social sciences" (p. 174). The Likert-type scales deal with attitudes and tend to have high reliabilities. The headings in a Likert scale usually are characterized by headings such as; strongly agree, agree, disagree, and strongly disagree. Likert scales apply a rating scale that spans spectrums from one extreme to the other. At one end of the scale, a very positive heading is in place, and the band crosses to the other extreme with a very negative heading.

This survey requires the principals to self-report along a continuum of choices (Gay, et al., 2006). To help ensure construct validity, reverse construction was used on five items out of thirty-one possible. According to Vogt (2005) construct validity is "the extent to which variables accurately measure the constructs of interest. In other words, how well are the variables 
operationalized? Do the operations really get at the things you are trying to measure?" (p. 58). The reverse items were brought to the principal's attention by using negative words and being in bold print (Suskie, 1996).

When the survey was completed, each domain included declarative statements pertaining to each item in the ISLLC standards. The respondent would be asked to indicate the number on the scale that best describes their perception of the statement in its relationship to them and their school. Each item on the instrument is scored on a scale from 1 to 4.

\section{Panel of Reviewers}

The survey instrument is the result of feedback from a group of individuals generally viewed as experts in the field of education (Gay, et al., 2006). All reviewers expressed interest and were supportive in their comments on needed changes for the instrument. These reviews constitute evidence of content validity of the Principal Perception Survey instrument.

The Reviewer One is currently a professor of education and served as a co-facilitator of the Danforth supported principals' preparation program. This reviewer expressed two concerns and an additional line of thought. The reviewer expressed a concern about the use of the two words "and" and "or" in the survey. The use of these terms created a double-barreled item for the principal to evaluate. The issue created by double-barreled items is which part of the question the person is going to answer. This feedback resulted in changing items to rid the survey of double-barreled questions. The second concern of the first reviewer dealt with the number response in the survey. In the original survey, there was a numbering system from 1-5 with the number three representing the not sure response. The recommendation from this reviewer was to remove the number three response and the section not sure forcing the principals to respond to the question. This forced response will cause the administrator to choose a 
direction along the continuum in the survey. The recommendation was accepted, and the original number three response was removed creating a numbering system of 1-4 with a forced response. An additional thought that Reviewer One suggested was to think about the variable as a continuum. The respondents' perceptions are going to fall along a band. The continuum or band in this case is all of the school leaders' beliefs in this survey about the ISLLC standards. With the survey having no place, for not sure criteria to be entered the continuum of school leaders' beliefs will fall into a range.

Reviewer Two has served as the chair of a teacher education preparation program and as a higher education administrator. In addition, Reviewer Two has served as a state-level evaluator of teacher education preparation programs. Reviewer Two pointed out some clutter in the directions of the survey. If the respondents get lost in the directions, the return rate might be affected and the data will be limited. Upon reviewing the parts of the instructions causing confusion, changes were made, and portions of the directions were reworked. Other parts of the directions were removed to provide clarity for the respondents. This reviewer had no additional comments, but provided supportive comments about the survey's direction.

Reviewer Three has experiences in teaching at the elementary, middle, and college level. In addition, reviewer three has served as an assistant principal and principal. The reviewer gave me suggestions on a couple of changes and recommended some additional readings and individuals I should contact. Reviewer Three suggested some changes to the survey questions to ensure clarity. The changes were made to the questions to provide a query that every potential respondent will interpret clearly in the same manner. In addition, other contacts were suggested as well as supplementary literature to be reviewed. Contacts were made and additional literature was reviewed during the instrument development. 
Reviewer Four is a current professor in a Department of Educational Leadership program at a school of higher education. This reviewer has served as the Executive Director of the National Council of Professors of Educational Administration and served as the Project Director of the Connexions Knowledge Base Project. Reviewer Four commented on the option Not Sure on the survey questionnaire. By taking out this option, the principal will be forced to respond. Additional comments by reviewer four recommended some additional literature for review concerning the ISLLC standards and the contact information of another expert in the field. This reviewer encouraged me to be aware of content validity during the process. "A measure has content validity when its items accurately represent the thing (the "universe") being measured. Content validity is not a statistical property; it is a matter of expert judgment" (Vogt, 2005, p. 59). The survey questions were submitted to a variety of experts during the design of the instrument.

Reviewer Five has thirteen years of public school experience. Currently this reviewer serves as a higher education faculty member. This reviewer has worked in the areas of teacher inquiry, professional learning communities, and school improvement. Reviewer Five's research has appeared in such journals as Teachers College Record and Journal of Teacher Education. Reviewer Five made some suggestions on different ways to complete the leadership survey. After further discussion, a decision was made to stay with the plan of using the self-report process for the principals. The main points of the discussion for further use could include many viewpoints on the principal's position. If the construction of this instrument is sound, many new possibilities will be present for future research. Later, future studies could expand to a 360degree survey instrument. For example, question one in the instrument could address teacher's perceptions covering the same content. Question one in this alternative instrument could be, my 
principal developed a shared vision as a good collaborator. Next, the instrument could move to superintendents and change question one to, I believe principal $\mathrm{X}$ builds and shares his/her vision collaborative. Then, the survey could move on to parents, and the possibilities are unlimited if there is a solid instrument in place. At the end of the study, all stakeholders would be included in a 360-degree survey.

Reviewer Six is a university professor with a background in statistics and has at least sixteen publications over the past five years on topics such as, learning, memory, and rehabilitation. The recommendations I received from reviewer number six included comments on match-up issues between Domain Questions and Standard Functions on three different survey queries. The adjustments were made to all three questions. The reviewer emphasized the importance of deciding specifically what information the investigator is trying to obtain, and eliminating anything unrelated. These recommendations were acted upon accordingly.

Reviewer Seven served as a middle school principal, assistant principal, guidance counselor, and teacher. In addition, this reviewer served as the director of the National Association of Secondary School Principals. This reviewer suggested additional resources pertaining to the ISLLC standards for me to view during the creation of the instrument for this study. This reviewer had no additional comments, but provided supportive comments about the survey's direction.

\section{Data Analysis}

A Likert scale has been used in the general field of social science research. The survey instrument in the data collection for this study has four points in its scale for each of the 31 items. The mid line point found in five-point Likert scales will not be present. Many times this response located in the center is a no opinion option. This scale uses a forced response to put the 
respondent at a data point along a range of perceptions. Because of a four-point scale, each principal's response will span a spectrum from one extreme to the other (Suskie, 1996). Each question on the survey has a rating scale. The headings range from Strongly Disagree to Strongly Agree. Thus, the four options for responding to each of the 31 items are:

1. Strongly Disagree,

2. Disagree,

3. Agree,

4. Strongly Disagree.

Reverse scoring is used on five of the items. The distance between each point on the scale has the same value moving along the continuum. For example, agree and disagree responses have the same distance in perception value due to the design of the scale. If an individual has a strong conviction, they have the option to strongly agree or strongly disagree.

The data will be divided into two camps. Both of the groups in this study will be selfselected. This will occur on the first line of the survey when the principal checks Professional Development School (PDS) or non-Professional Development School. From this point, the survey applications will be disaggregated into units of analysis. The characteristic dividing the two groups will be its connection with PDS-schools. Additional identifiers will be in place. Each respondent will select gender and number of service years.

Each item on the survey will receive a $t$-test to determine the difference of the two means between the two groups. In addition, a $t$-test will be completed on each of the survey domains. This study includes the following six Domains: Visioning (5 items), Advocating (9 items), Management (5 items), Collaboration (4 items), Ethics (5 items), and Community Relations (2 items). 


\section{Survey Questionnaire Dissemination and Collection}

Sending questionnaires by e-mail is fast and efficient. However, as in any method, it has both advantages and disadvantages, and for the most part can be comparable to questionnaires delivered by mail (Gay, et al., 2006). In this case, the speed and ease of completing an on-line survey serves as an advantage. The disadvantage is the technological part of the survey excluding individuals who decline to use e-mail. The majority of principals in this era of technology use e-mail on a daily basis with their job. This study proposes using the following steps to gather the survey information.

Step 1 is the initial contact in which an advance e-mail will be sent to each potential survey respondent. This e-mail will establish a connection with the principal before the survey arrives, with background history of the survey and contact information for any questions. In addition, a letter of support (see Appendix B) for this survey will be requested from state leaders. Suskie (1996) recommends personalizing the contact with the respondent by including contact information and offering to send a copy of the survey results.

Step Two will involve the use of an online survey tool to disseminate and collect the responses. The researcher recognizes that principals filling out this survey are extending a great favor. To show appreciation for their effort, the goal will be to minimize the respondent's trouble and make accessibility a top priority. Easy access and a trouble free set-up of the survey will present a better chance of the participant completing the survey (Suskie, 1996). Survey Monkey will be the tool used to facilitate the survey process. A special tab will be inserted within the e-mail to provide a one-click tab to reach the survey page within Survey Monkey for easy access. The responses will be collected from Survey Monkey and placed in a spreadsheet. 
The spreadsheet will then be imported to the Statistical Package for the Social Sciences (SPSS) 16.0 family program for analysis.

Step Three will involve the use of reminders through an additional e-mail sent to nonrespondents of the first survey (see Appendix E). As the surveys arrive, a spreadsheet will display the progress of returns from principals. This will help to ensure reminders are only sent to non-respondents. This additional e-mail will restate the importance of the study and the need for their valuable feedback. In addition, an option will be offered to any participant desiring to receive a paper copy of the survey.

Step Four will involve the final process of reminders. A telephone call to request nonrespondents to answer the questionnaire will be completed. The issue in this case will be the availability of principals to take phone calls. In some cases, only messages of reminders will be an option.

What is an acceptable response rate for this study? According to Suskie (1996), an emphasis must be placed on the quality of the survey responses.

One of the most common questions asked by novice researchers is, "What percentage of my questionnaires do I need to get back?" There are both simple and complex answers to this question. The simple answer is that most pros suggest a $70 \%$ to $80 \%$ response rate and consider $50 \%$ minimally adequate. The complex answer is that this is almost an irrelevant question for two reasons. The first reason is that, as any good researcher will tell you, quality of responses is more important than quantity. It is more important to have respondents who are representative of the group from which you are sampling than to have a large return rate. (p. 69) 
The following is a timetable for the e-mail survey to be conducted with school principals with valid e-mail addresses in the state of West Virginia.

- Day 1 (Monday) Send out an advance e-mail explaining the survey process (see Appendix C).

- Day 2 (Tuesday) E-mail initial questionnaire package (see Appendix D).

- Days 3 and 4 (Wednesday and Thursday) Surveys are received by principals.

- Days 4 and 5 (Thursday and Friday) Returns begin coming in.

- Day 8 (Monday) E-mail survey reminders to all non-respondents (see Appendix E).

- Days 9, 10, 11, and 12 (Tuesday, Wednesday, Thursday, and Friday) Check incoming surveys to identify non-respondents.

- Day 15 (Monday) E-mail second questionnaire package (see Appendix F).

- Day 16, 17, 18, and 19 (Tuesday, Wednesday, Thursday, and Friday) Check incoming surveys to identify non-respondents

- Day 22 (Monday) E-mail the final reminder (see Appendix G).

Summary of research methods. Survey research is the method proposed to answer the research questions. Is there a relationship between involvement with a professional development school and how leaders perceive their work in alignment with the ISLLC standards? In addition, will PDS principals have higher scores as opposed to non-PDS principals on an instrument designed to test perceptions in alignment with the ISLLC standards?

The survey instrument design was based on the Interstate School Leaders Licensure Consortium (ISLLC) standards. This survey used equivalent statements from the (ISLLC) standards as a basis for all items designed for the principals' responses. The Principal Perception Survey contains 31 items. This instrument was reviewed by seven knowledgeable educators. 
The population of this proposed study will come from a convenience sampling of public school principals with e-mail addresses from schools in West Virginia. The proposed survey will look at the results of the data in aggregate form. An aggregate is "a group of persons, or other units of analysis, that have certain traits or characteristics in common without necessarily having any direct social connection with one another, such as the population of a city. Also called aggregation" (Vogt, 2005, p. 5). The samples drawn from the population will be divided into two groups. The first group, or Control Group, will be the principals from non-Professional Development Schools. Group 2, or the Experimental Group, will be the principals from Professional Development Schools.

After an advance e-mail has been sent to survey participants, the gathering of data will begin. Survey Monkey will be the tool used to facilitate the survey process. The responses will be collected from Survey Monkey and placed in a spreadsheet. The spreadsheet will then be imported to the Statistical Package for the Social Sciences (SPSS) 16.0 family program for analysis.

A t-test will be used on each survey item to determine the difference of the two means between the two groups. In addition, a $t$-test will be completed on each of the survey domains. The results of the $t$-test will be applied to the null and alternative hypotheses. The null hypothesis states that there is no statistically significant difference in the domain's scores when comparing principals who work in a Professional Development School as opposed to nonProfessional Development School principals in their perception of their work in accordance with the ISLLC standards. The alternative hypothesis states that there is a statistically significant difference in the domain scores when comparing individuals in the two groups being studied. 
Additionally, Means and Standard Deviations will be presented for each Item and each Domain for the two groups. Tables and figures will be presented as appropriate. The rejection or acceptance of the null hypothesis is based on the significance level of .05 as the criterion. This suggests a $95 \%$ probability that the difference was due to the experimental treatment rather than to a sampling error. 


\section{Chapter Four: Analysis of Results}

This chapter presents the analysis of the data collected to answer the question: "Is there a relationship between involvement with a Professional Development School and how leaders perceive their work in accordance with the ISLLC standards?" The principals selected for this study come from a convenience sample from public county schools within West Virginia. All of the principals surveyed had an accessible public e-mail address in West Virginia. A series of emails with an instrument specifically designed for collecting the data was sent to the principals with active e-mail addresses (Gay, Mills, \& Airasian, 2006). This allowed data to be collected within a relatively short period. Within West Virginia, 575 e-mail addresses of principals were active. Of the 575 e-mail addresses of principals, 249 principals responded. The return rate was 43.3\%. Of the 249 responses, 132 responses were from non-Professional Development School principals, and 117 responses were received from Professional Development School principals. The response rate was 53\% from non-PDS principals compared to $47 \%$ from PDS principals.

The first section of chapter Four contains the descriptive and statistical analysis of survey responses by domain of both of the comparison groups (132 non-PDS principals versus 117 PDS principals). The treatment group consisted of principals working within a Professional Development School. The second section of chapter Four describes the descriptive and statistical analysis of the responses to each of the 31 survey questions by both groups.

The final section describes the narrative responses of both groups to the following two questions:

(1) How have the Interstate School Leaders Licensure Consortium standards (ISLLC) affected your leadership? 
(2) What do you believe are the greatest challenges for providing a culture of professional renewal inside a Professional Development School system?

The $t$-test is the statistical calculation that determines whether the means of the responses of the comparison group and the treatment group are significantly different at a probability level of .05. In determining significance, the $t$-test makes adjustments for the fact that the distribution of scores for small samples becomes increasingly different from the normal distribution. Even if the null hypothesis were true, the two sample means would not be expected to be identical. There will be some chance variation. The $t$-test for the two independent samples is a parametric test of significance that determines whether the observed difference is sufficiently larger than the difference that would be expected solely by chance (Gay, et al., 2006).

Also used in this analysis is the calculation of the effect size, representing the power of the treatment. The effect size is expressed as a decimal number, and although numbers greater than 1.00 are possible, they do not occur very often. An effect size near .00 means that, on average, experimental and comparison groups performed the same. A positive effect size would means that, on average, the experimental group's (PDS principals) perceptions were more closely aligned with the ISLLC standards than the non-PDS principals were. A negative effect would suggest the opposite. As the number of participants in a research study increases, so does the accuracy of the data. Although there are no hard and fast rules, it is generally agreed that an effect size in the twenties (e.g., .28) indicates a treatment that produces a relatively small effect. An effect size in the eighties (e.g., 81) indicates a powerful treatment (Gay, et al., 2006).

Gay, Mills, and Airasian (2006) recommend a Likert-type scale that groups items into clusters that address the same issue and develop total scores across an item cluster. The Principal Perception Survey was designed on this basis. The instrument consisted of 31 
questions or statements. The 31 items were clustered to correspond to the six ISLLC standards. The six domains, which correspond to the six ISLLC standards listed in the survey instrument, are:

(1) Visioning

(2) Advocating

(3) Management

(4) Collaboration

(5) Ethics

(6) Community Relations

The null and alternative hypotheses for each of the six domains are:

$\mathrm{H}_{0}$ : There is no statistically significant difference in Domain $\underline{\mathrm{X}}$ scores when 9comparing individuals who are principals of Professional Development Schools with those who are principals of non-Professional Development Schools.

$\mathrm{H}_{1}$ : There is a statistically significant difference in Domain $\underline{\mathrm{X}}$ scores when comparing individuals who are principals of Professional Development Schools with those who are principals of non-Professional Development Schools.

\section{Descriptive and Statistical Analyses by Domain}

Of the 587 principals asked to participate in the survey, 249 responded. Of this number, 249 completed the survey, creating a response rate of 43.3 percent. The respondents of the comparison group (non-Professional Development School Principals) consisted of 66 males (50 percent of the comparison group) and 66 females (50 percent). The respondents of the treatment group (Professional Development School Principals) consisted of 69 males (59 percent) and 48 females (41 percent). 
Overall, 249 principals responded to the survey. The difference in the number of participants between the treatment group and the comparison group is negligible. The treatment group had 117 responses. The comparison group had 132 responses. One county in West Virginia was not represented due to non-participation in the survey.

Domain 1: Visioning (ISLLC standard 1). An educational leader promotes the success of every student by facilitating the development, articulation, implementation, and stewardship of a vision of learning that is shared and supported by all stakeholders.

The analysis of the respondents' scores for Domain 1 revealed no statistical difference between the responses of the principals from Professional Development Schools versus principals from non-Professional Development Schools.

Domain 1 contains the cluster of the following five questions:

1. I consider myself a good collaborator when it comes to developing a shared vision for this school.

2. I do not collect and use data to identify goals or assess organizational effectiveness.

3. I consider myself a methodical planner when it comes to the development of school goals.

4. I often deliver the message that continuous improvement is necessary to achieve goals. 5. I often include stakeholder groups when assessing whether or not we have achieved goals that move us toward the shared vision.

The 132 respondents in the comparison group secured a mean score of 3.43 and a standard deviation of 0.572 . The 117 respondents in the treatment group obtained a mean score of 3.45 and a standard deviation of 0.609 . Utilizing $p>.05$ (non-significant), the $t$-Test showed 
a two-tailed $t$-score of 0.621 . The analysis failed to reject the null hypothesis. There is no statistically significant difference between the scores of the comparison group and the treatment group (PDS) in Domain 1: Visioning (see Figure 1).

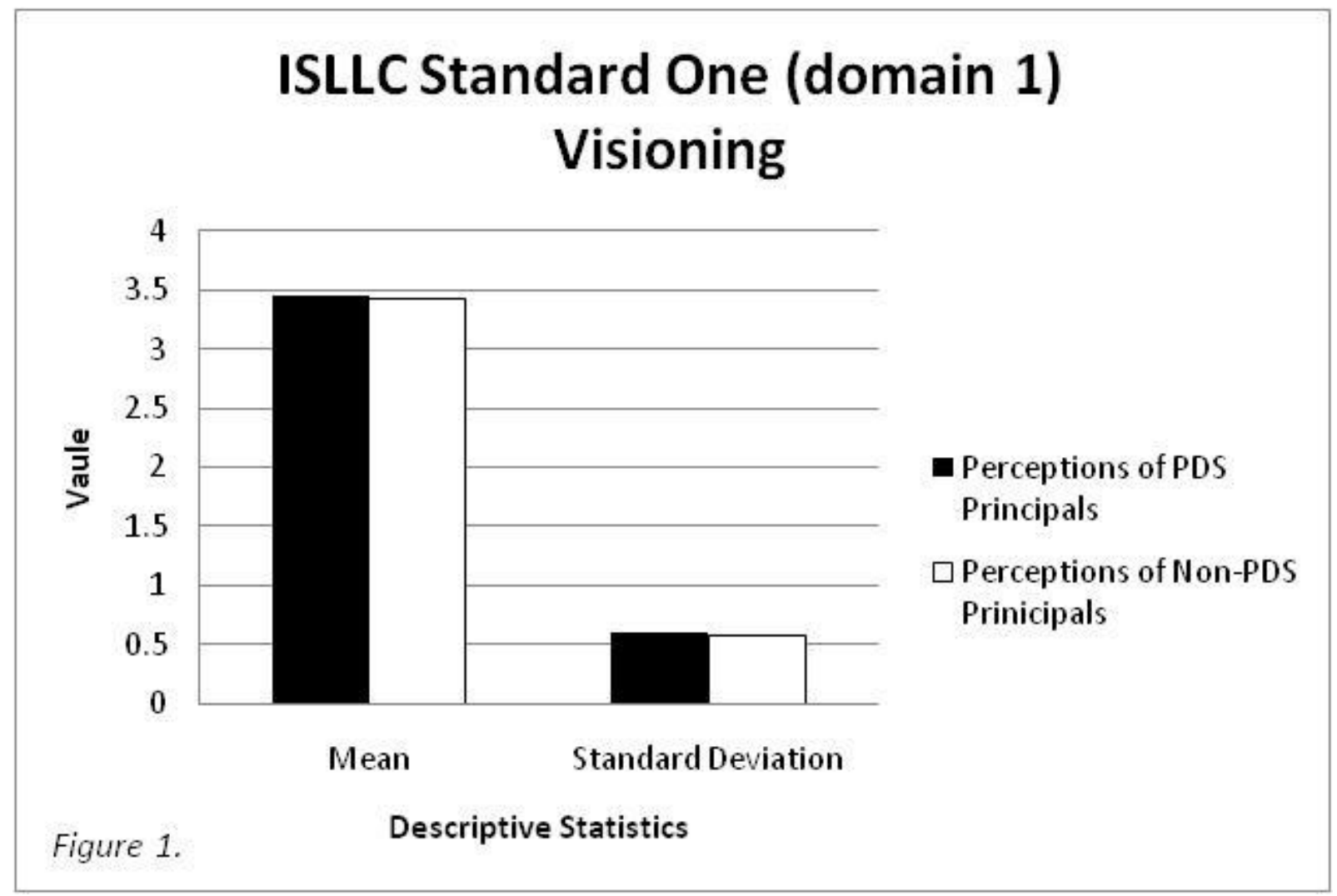

Domain 2: Advocating (ISLLC standard 2). An education leader promotes the success of every student by advocating, nurturing, and sustaining a school culture and instructional program conducive to student learning and staff professional growth.

The analysis of the respondents' scores for Domain 2 showed that there was no statistical difference between the responses of the principals from Professional Development Schools and the responses of the principals from non-Professional Development Schools. The explanation of this analysis follows. Domain 2 contains the cluster of the following nine questions:

1. My faculty and I have developed and maintained high expectations for all students. 
2. A rigorous curricular program has been collaboratively developed and is reviewed annually.

3. I believe we have a structured motivational learning environment at this school.

4. I have a plan for supervising instruction and I follow it.

5. I do not believe our current assessment system is structured for student growth.

6. I believe it is important to develop the instructional capacity of staff.

7. My faculty, staff, and I value and protect instructional time.

8. We are constantly engaged in discussions about the most effective instructional technologies, and we seek ways to support getting and using it.

9. Evaluation of instructional program impact is done continuously and the program is modified daily to meet students' needs.

Domain 2 contained nine questions. The number of comparison group respondents was 132 with a mean score of 3.32 and a standard deviation of 0.667 . The number of treatment group respondents was 117 with a mean score of 3.37 and a standard deviation of 0.670 . [The $t$-Test showed a two-tailed $t$-score of $1.967 p>.05$ (non-significant).] The analysis failed to reject the null hypothesis. There is no statistically significant difference in the scores of the comparison group and the treatment group in Domain 2 (see Figure 2). 


\section{ISLLC Standard Two (domain 2) Advocating}

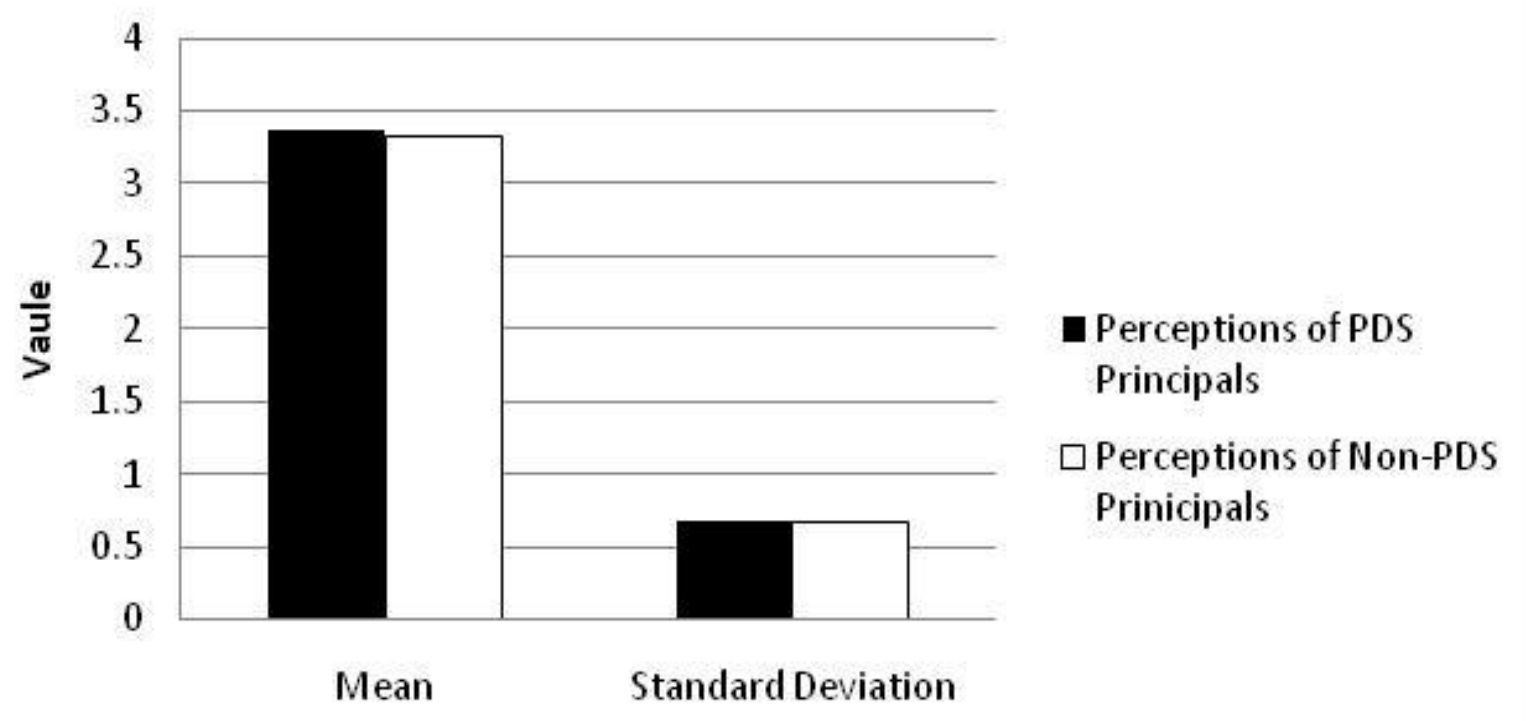

Figure 2.

Descriptive Statistics

Domain 3: Management (ISLLC standard 3). An education leader promotes the success of every student by ensuring management of the organization, operation, and resources for a safe, efficient, and effective learning environment.

The analysis of the respondents' scores for Domain 3 disclosed no statistical difference between the responses of the principals from Professional Development Schools and the principals from non-Professional Development Schools. The explanation of this analysis follows. Domain 3 contains the cluster of the following five questions:

1. I constantly review operational systems to determine if they are meeting the needs of all stakeholders.

2. I challenge my faculty and staff to find ways to maximize resources. 
3. Safety policy and procedures are at the forefront of continuous review.

4. I develop leadership capacity in my faculty and staff by enabling them to solve organizational problems.

5. Focused teaching and learning time is built into logistics.

Domain 3 contained five questions. The number of comparison group respondents was 132 with a mean score of 3.38 and a standard deviation of 0.556 . The number of the treatment group respondents was 117 with a mean score of 3.42 and a standard deviation of 0.540 . [The $t$ Test showed a two-tailed $t$-score of $1.187 p>.05$ (non-significant).] The analysis failed to reject the null hypothesis. There is no statistically significant difference in the scores of the comparison group and the treatment group in Domain 3 (see Figure 3).

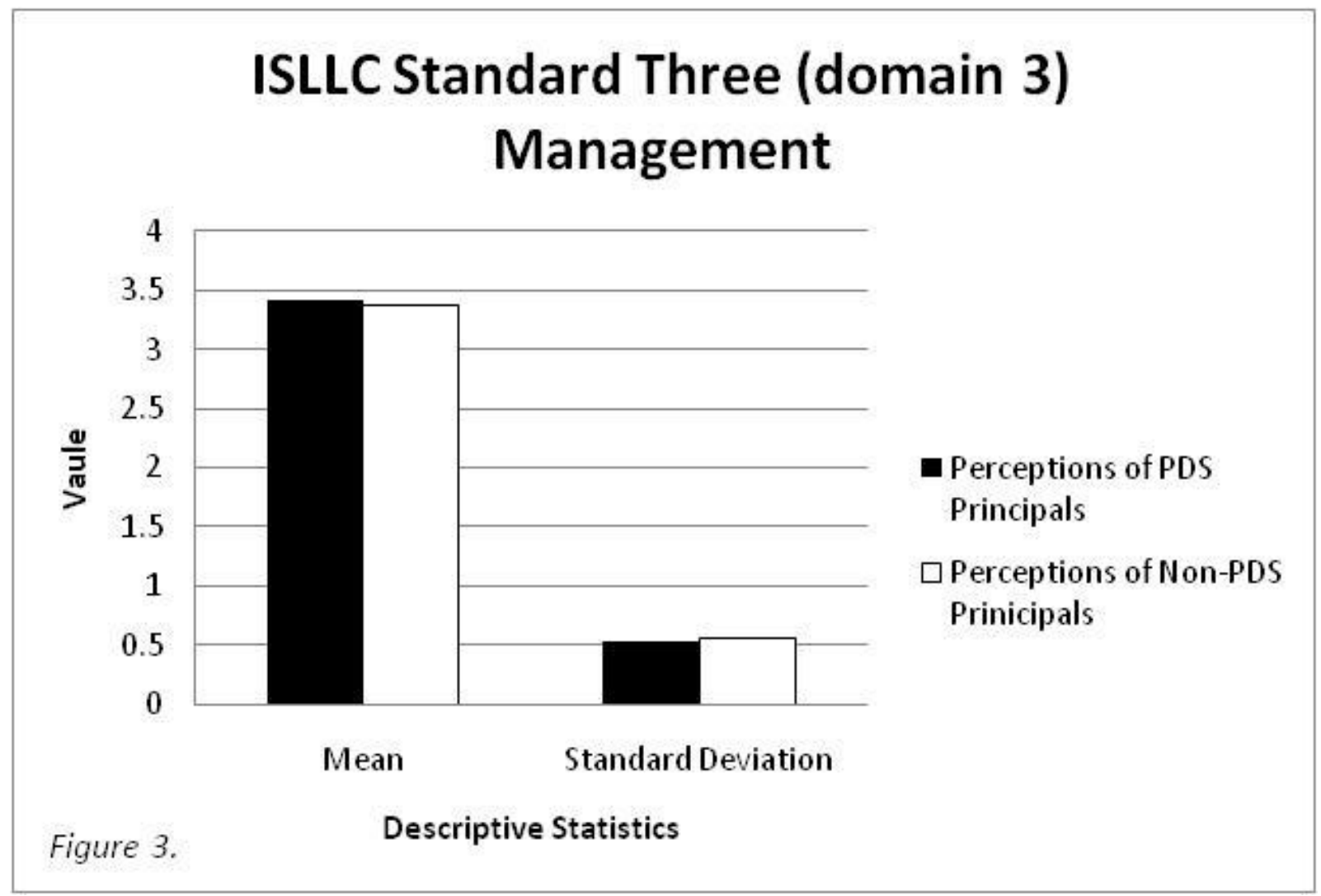


Domain 4: Collaboration (ISLLC standard 4). An education leader promotes the success of every student by collaborating with faculty and community members, responding to diverse community interests and needs, and mobilizing community resources.)

The analysis of the respondents' scores for Domain 4 revealed no statistical difference between the responses of the principals from Professional Development Schools and the principals from non-Professional Development Schools. The explanation of this analysis follows. Domain 4 contains the cluster of the following four questions:

1. The faculty and I analyze data pertinent to the educational environment.

2. I expect faculty and staff members to welcome and engage the community.

3. The well-being of students and their families give us a common purpose for building positive relationships.

4. I don't waste my time reaching out to disinterested community partners.

Domain 4 contained four questions. The number of comparison group respondents was 132 with a mean score of 3.44 and a standard deviation of 0.622 . The number of the treatment group respondents was 117 with a mean score of 3.43 and a standard deviation of 0.636 . [The $t$-Test showed a two-tailed $t$-score of $-0.195 p>.05$ (non-significant).] The analysis failed to reject the null hypothesis. There is no statistically significant difference between the scores of the comparison group and the treatment group in Domain 4 (see Figure 4). 


\section{ISLLC Standard Four (domain 4) Collaboration}

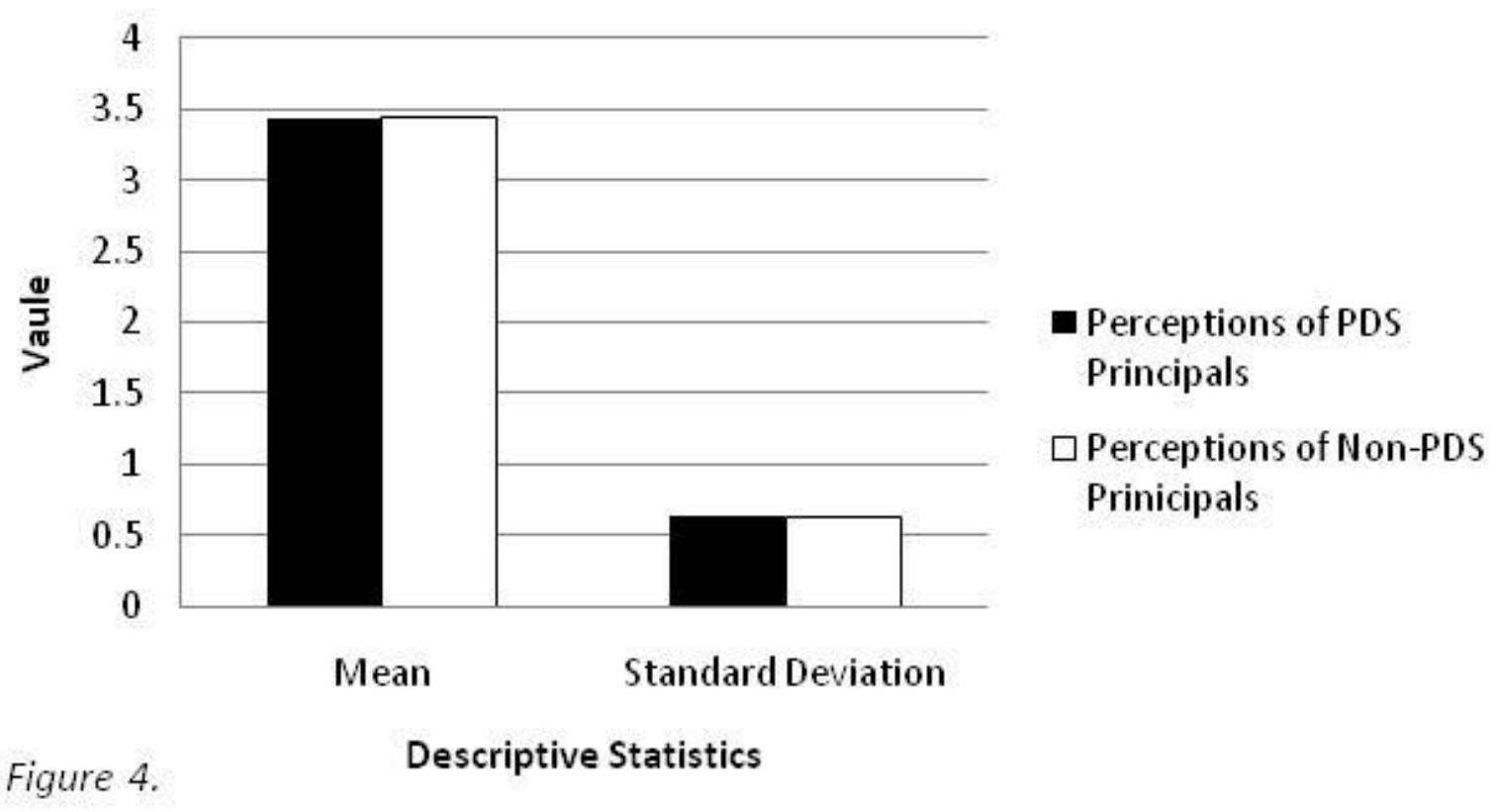

Domain 5: Ethics (ISLLC standard 5). An education leader promotes the success of every student by acting with integrity, fairness, and in an ethical manner.

The analysis of the respondents' scores for Domain 5 showed no statistical difference between the responses of the principals from Professional Development Schools and the principals from non-Professional Development Schools. The explanation of this analysis follows. Domain 5 contains the cluster of the following five questions:

1. I do not think it is important that every student maximize his/her achievement or success.

2. I model the kind of behavior I expect from everyone else.

3. My vision for building a fear free culture of teaching and learning is centered by democratic principles, equality and fairness, and strength in diversity. 
4. Legal consequences of decision-making are not connected to moral decision making.

5. I base all my leadership work on opening the door of education to all people regardless of social status, ethnicity, gender, religion, and cultural background.

Domain 5 contained five questions. The number of comparison group respondents was 132 with a mean score of 3.45 and a standard deviation of 0.738 . The number of the treatment group respondents was 117 with a mean score of 3.43 and a standard deviation of 0.763 . [The $t$-Test showed a two-tailed $t$-score of $-0.305 . p>.05$ (non-significant).] The analysis failed to reject the null hypothesis. There is no statistically significant difference between the scores of the comparison group and the treatment group in Domain 5 (see Figure 5).

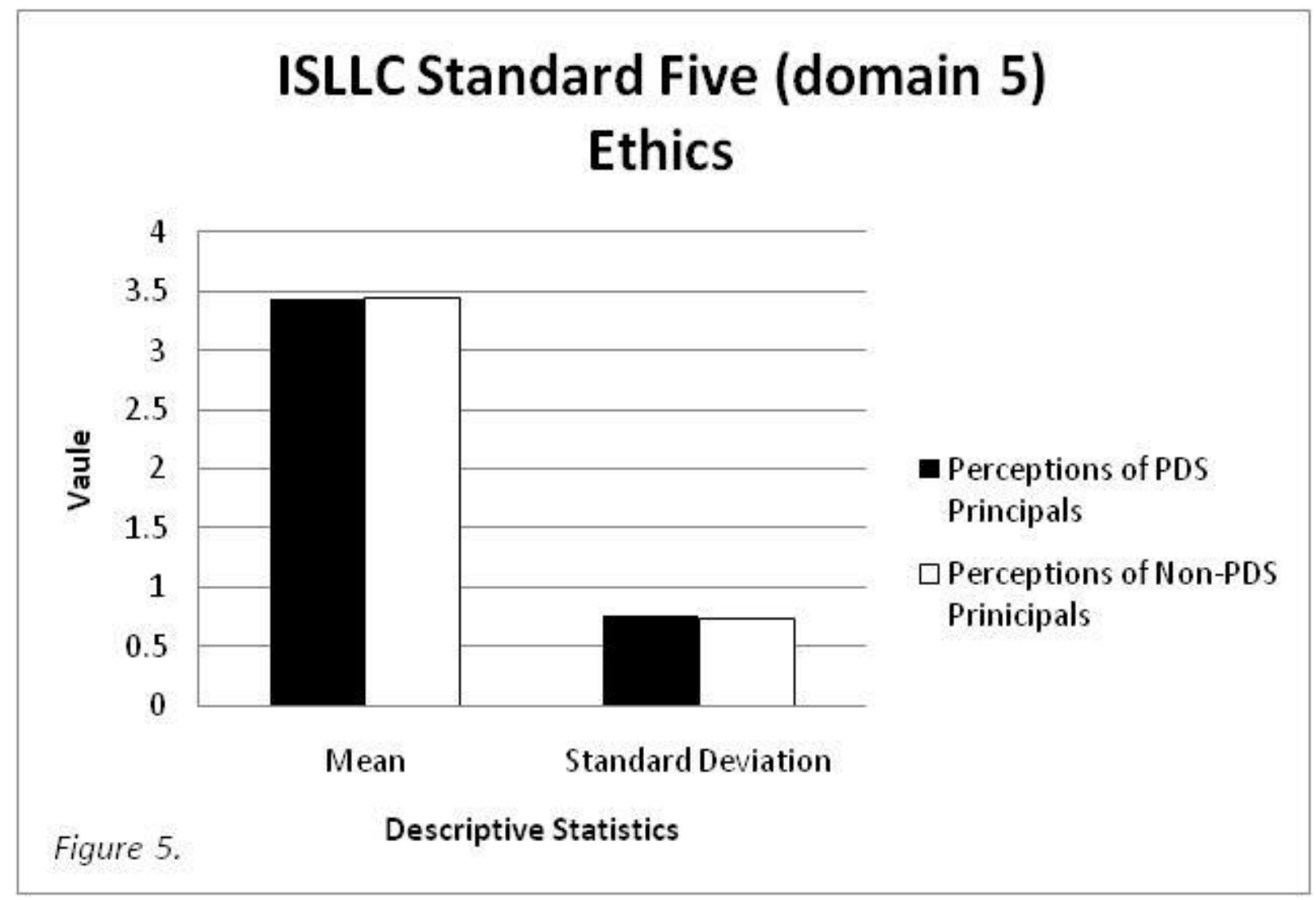


Domain 6: Community relations (ISLLC standard 6). An education leader

promotes the success of every student by understanding, responding to, and influencing the political, social, economic, legal, and cultural context.)

The analysis of the respondents' scores for Domain 6 showed that there was no statistical difference between the responses of the principals from Professional Development Schools and principals from non-Professional Development Schools. The explanation of this analysis follows. Domain 6 contains the cluster of the following three questions:

1. My faculty and I advocate for children and their families.

2. I act to influence policies affecting student learning.

3. I anticipate emerging trends and adapt my leadership strategies.

Domain 6 contained three questions. The number of comparison group respondents was 132 with a mean score of 3.49 and a standard deviation of 0.581 . The number of the treatment group respondents was 117 with a mean score of 3.48 and a standard deviation of 0.555 . [The $t$-Test showed a two-tailed $t$-score of $-0.108 . p>.05$ (non-significant).] The analysis failed to reject the null hypothesis. There is no statistically significant difference between the scores of the comparison group and the treatment group in Domain 6 (see Figure 6). 


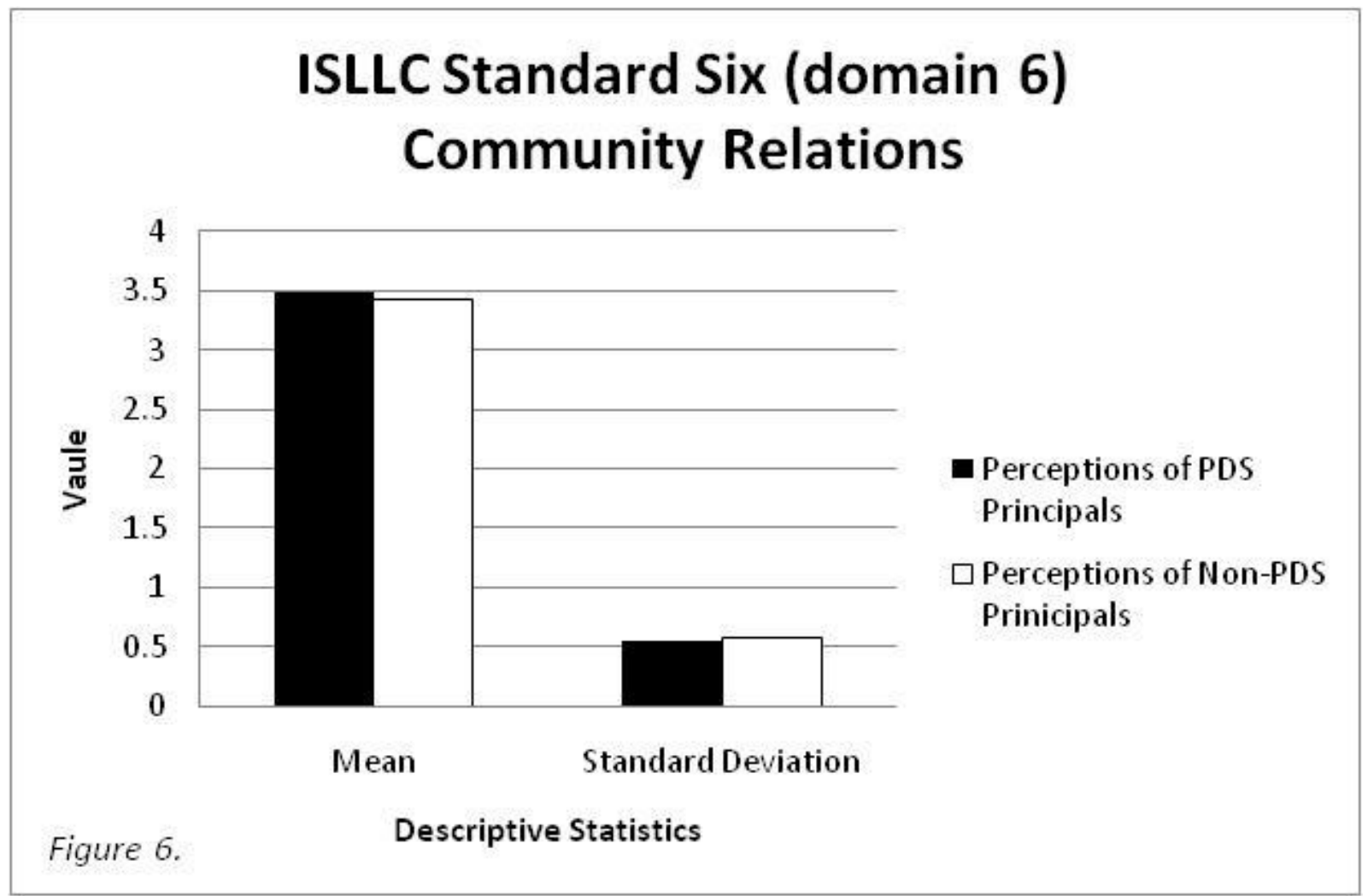

\section{Descriptive and Statistical Analysis by Item}

Of the 31 survey statements to which principals responded, there were statistically significant differences in one of them. The principals in the comparison group (NonProfessional Development Schools) had a higher mean score in nine items. The principals in the treatment group (Professional Development Schools) had a higher mean score in 22 items. An explanation of the statistical analysis of responses to each survey item follows.

\section{Survey Domain 1 (visioning).}

Visioning: Item 1. I consider myself a good collaborator when it comes to developing a shared vision for this school. 
The analysis of the respondents' scores for survey Item 1 showed no statistical difference between the responses of the principals who were involved as leaders in non-Professional Development Schools compared to principals as leaders in Professional Development Schools. The explanation of this analysis follows.

The comparison group $\mathrm{N}$ was 132 with a mean score of 3.50 and a standard deviation of 0.502. The treatment group $\mathrm{N}$ was 117 with a mean score of 3.48 and a standard deviation of 0.596. [The $t$-Test showed a two-tailed $t$-score. of $-.307 \mathrm{p}>.05$ (non-significant).] The analysis failed to reject the null hypothesis. There is no statistically significant difference between the scores of the comparison group and the treatment group in Domain 1: Item 1. The effect size, $d$ calculation, is 0.04 , indicating a small effect of the difference made by the treatment. The positive $d$ indicates that the comparison group scores were larger than the treatment group (see Figure 7).

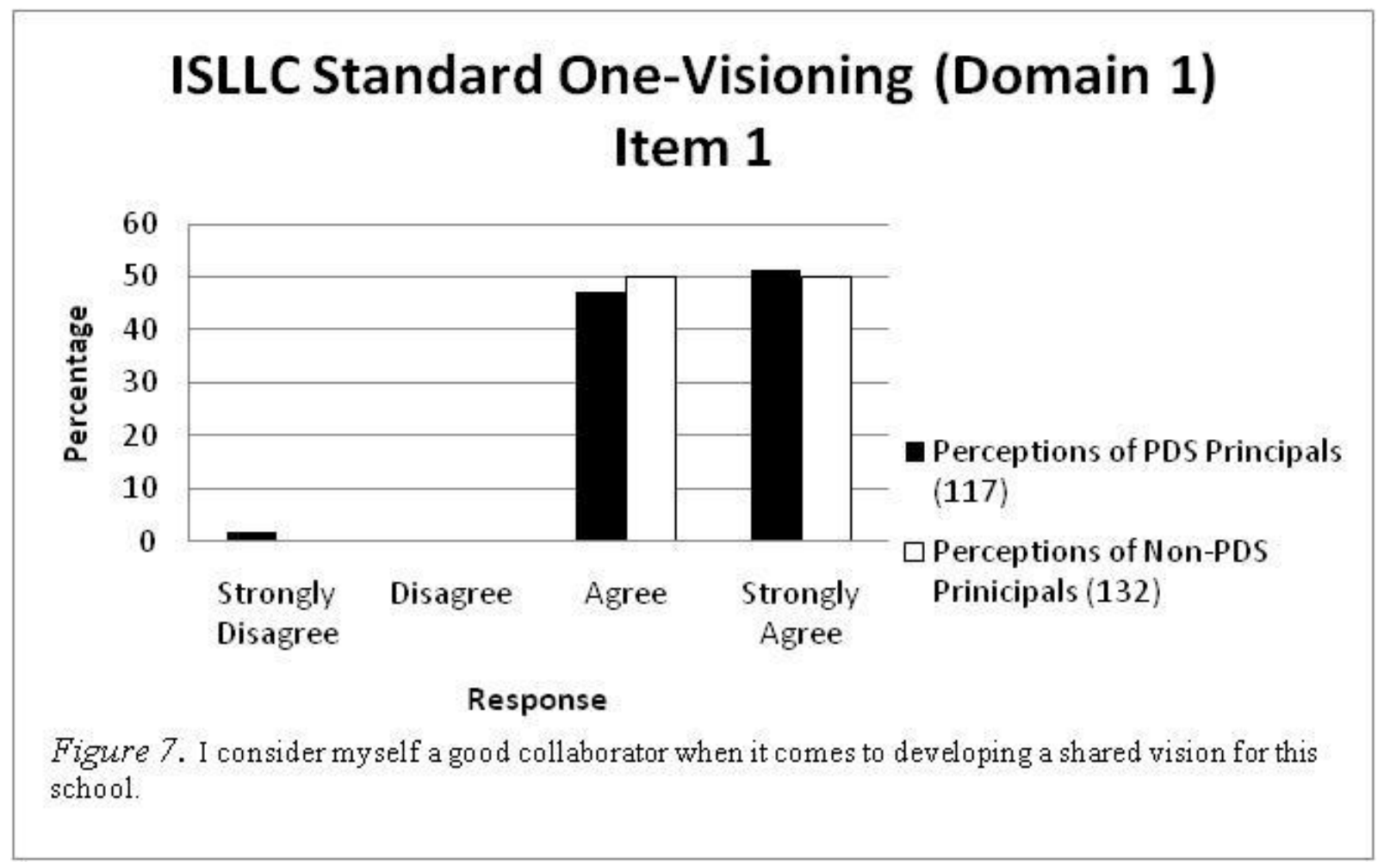


Visioning Item 2. I do not collect and use data to identify goals or assess organizational effectiveness.

The analysis of the respondents' scores for survey Item 2 showed that there was not a statistical difference between the responses of the principals who were involved as leaders in non-Professional Development Schools compared to principals as leaders in Professional Development Schools. The explanation of this analysis follows.

The comparison group $\mathrm{N}$ was 132 with a mean score of 3.70 and a standard deviation of 0.552. The treatment group $\mathrm{N}$ was 117 with a mean score of 3.65 and a standard deviation of 0.634. [The $t$-Test showed a two-tailed $t$-score of $-0.631 p>.05$ (non-significant).] The analysis failed to reject the null hypothesis. There is no statistically significant difference between the scores of the comparison group and the treatment group in Domain 1: Item 2. The effect size, $d$ calculation, is 0.08 , indicating a small effect of the difference made by the treatment.

The positive $d$ indicates that the comparison group scores were larger than the treatment group (see Figure 8). 


\section{ISLLC Standard One-Visioning (Domain 1) Item 2}

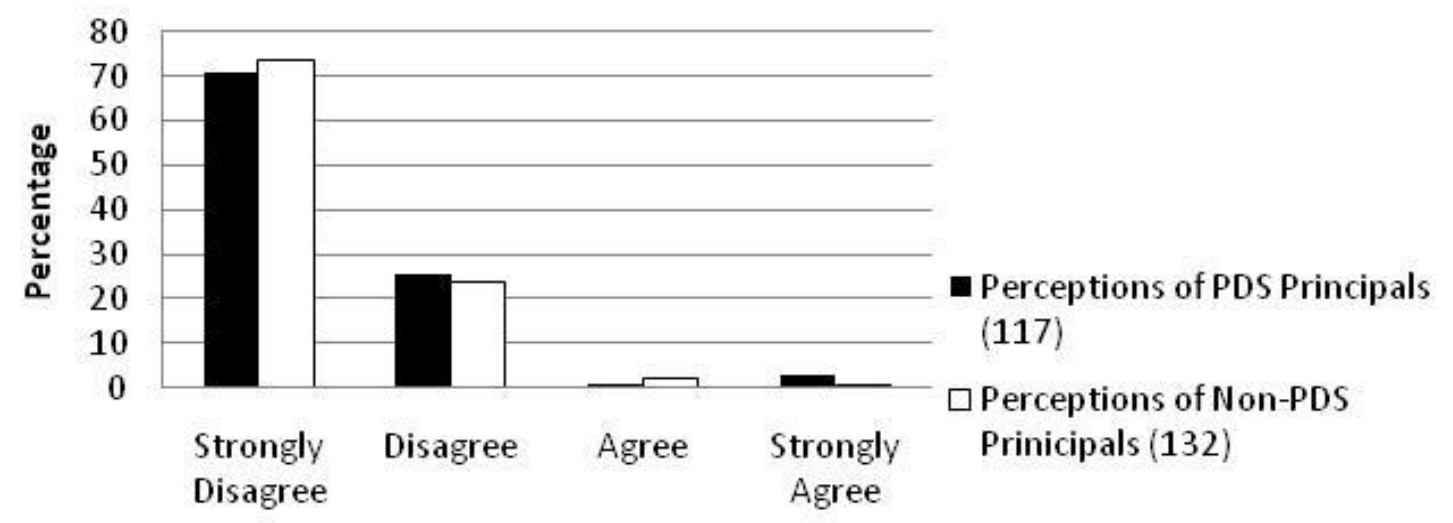

Response

Figure 8. I do not collect and use data to identify goals or assess organizational effectiveness.

Visioning Item 3. I consider myself a methodical planner when it comes to the development of school goals.

The analysis of the respondents' scores for survey Item 3 showed that there was not a statistical difference between the responses of the principals who were involved as leaders in non-Professional Development Schools compared to principals as leaders in Professional Development Schools. The explanation of this analysis follows.

The comparison group $\mathrm{N}$ was 132 with a mean score of 3.23 and a standard deviation of 0.518 . The treatment group $\mathrm{N}$ was 117 with a mean score of 3.21 and a standard deviation of 0.534 . [The $t$-Test showed a two-tailed $t$-score of $-0.332 p>.05$ (non-significant).] The analysis failed to reject the null hypothesis. There is no statistically significant difference between the scores of the comparison group and the treatment group in Domain 1: Item 3.

The effect size, $d$ calculation, is 0.04 , indicating a small effect of the difference made by 
the treatment. The positive $d$ indicates that the comparison group scores were larger than the treatment group (see Figure 9).

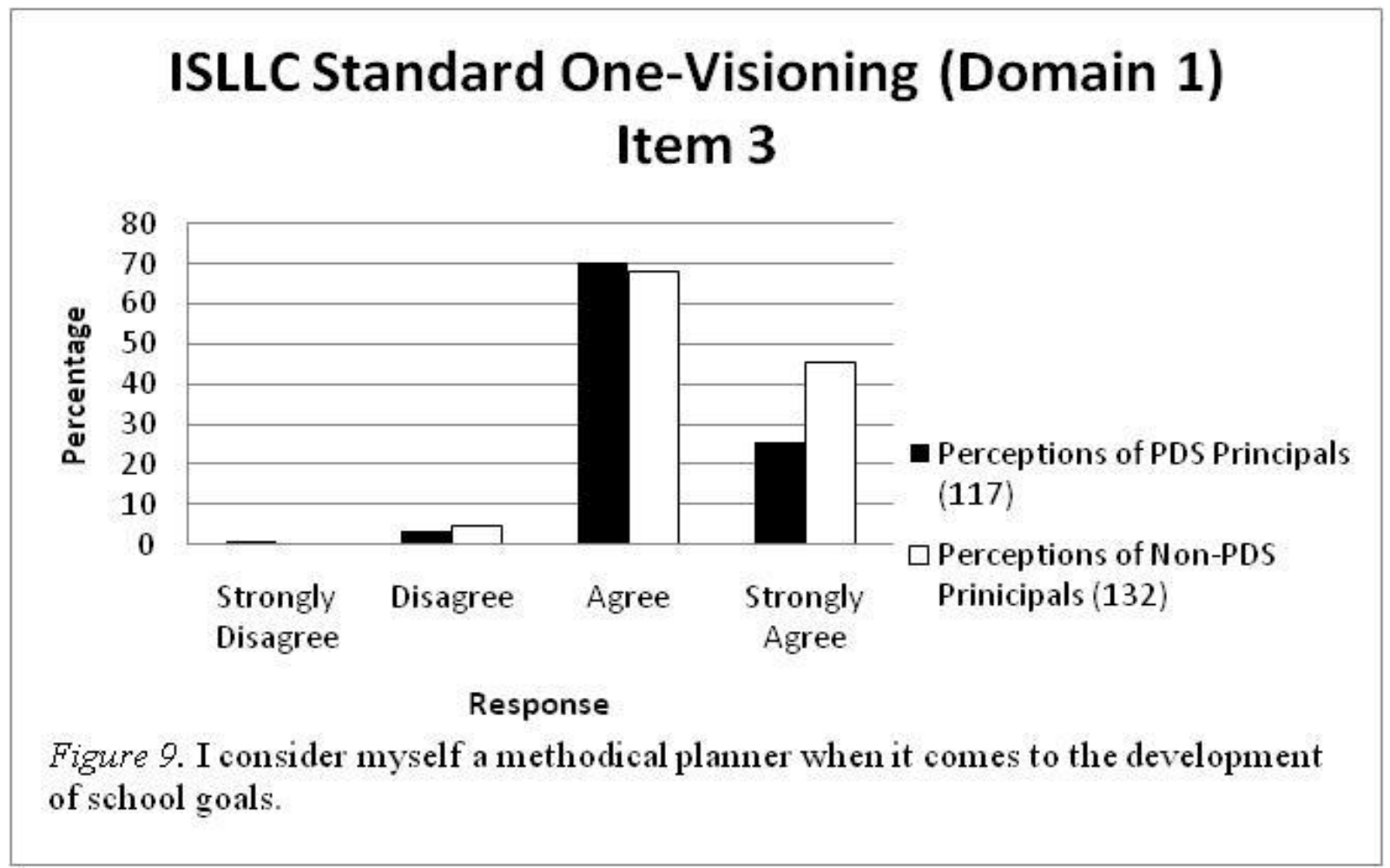

Visioning: Item 4. I often deliver the message that continuous improvement is necessary to achieve goals.

The analysis of the respondents' scores for survey Item 4 showed that there was no statistical difference between the responses of the principals who were involved as leaders in non-Professional Development Schools compared to principals as leaders in Professional Development Schools. The explanation of this analysis follows.

The comparison group $\mathrm{N}$ was 132 with a mean score of 3.47 and a standard deviation of 0.611. The treatment group $\mathrm{N}$ was 117 with a mean score of 3.54 and a standard deviation of 0.595. [The $t$-Test showed a two-tailed $t$-score of $0.897 p>.05$ (non-significant).] The analysis failed to reject the null hypothesis. 
The effect size, $d$ calculation, is -0.12 , indicating a small effect of the difference made by the treatment. The negative $d$ indicates that the treatment group scores were larger than the comparison group (see Figure 10).

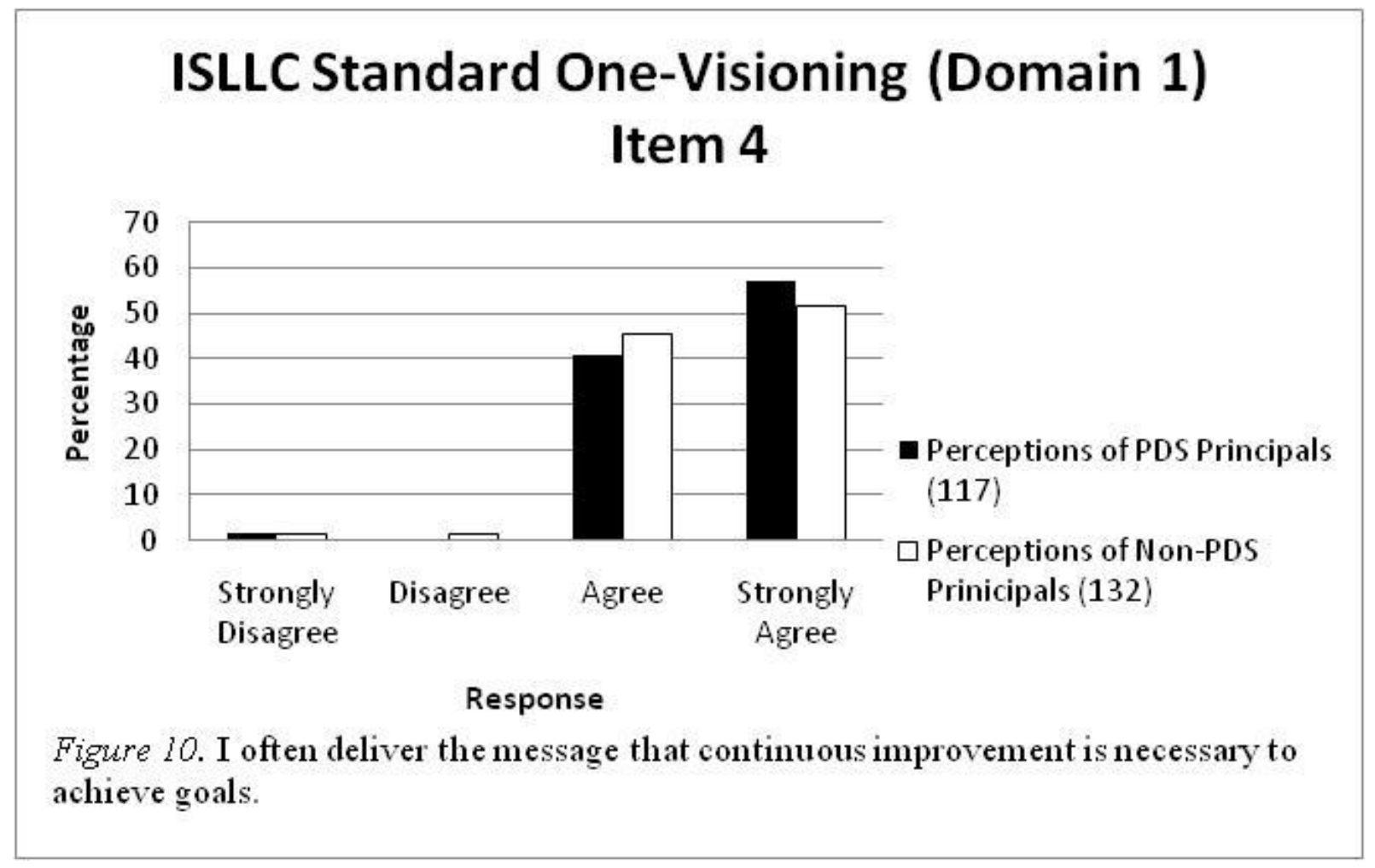

Visioning: Item 5. I often include stakeholder groups when assessing whether or not we have achieved goals that move us toward the shared vision.

The analysis of the respondents' scores for survey Item 5 showed that there was not a statistical difference between the responses of the principals who were involved as leaders in non-Professional Development Schools compared to principals as leaders in Professional Development Schools. The explanation of this analysis follows.

The comparison group $\mathrm{N}$ was 132 with a mean score of 3.25 and a standard deviation of 0.544. The treatment group $\mathrm{N}$ was 117 with a mean score of 3.38 and a standard deviation of 
0.598. [The $t$-Test showed a two-tailed $t$-score of $1.742 p>.05$ (non-significant).] The analysis failed to reject the null hypothesis. There is no statistically significant difference between the scores of the treatment group and the comparison group in Domain 1: Item 5.

The effect size, $d$ calculation, is -0.23 , indicating a small effect of the difference made by the treatment. The negative $d$ indicates that the treatment group scores were larger than the comparison group (see Figure 11).

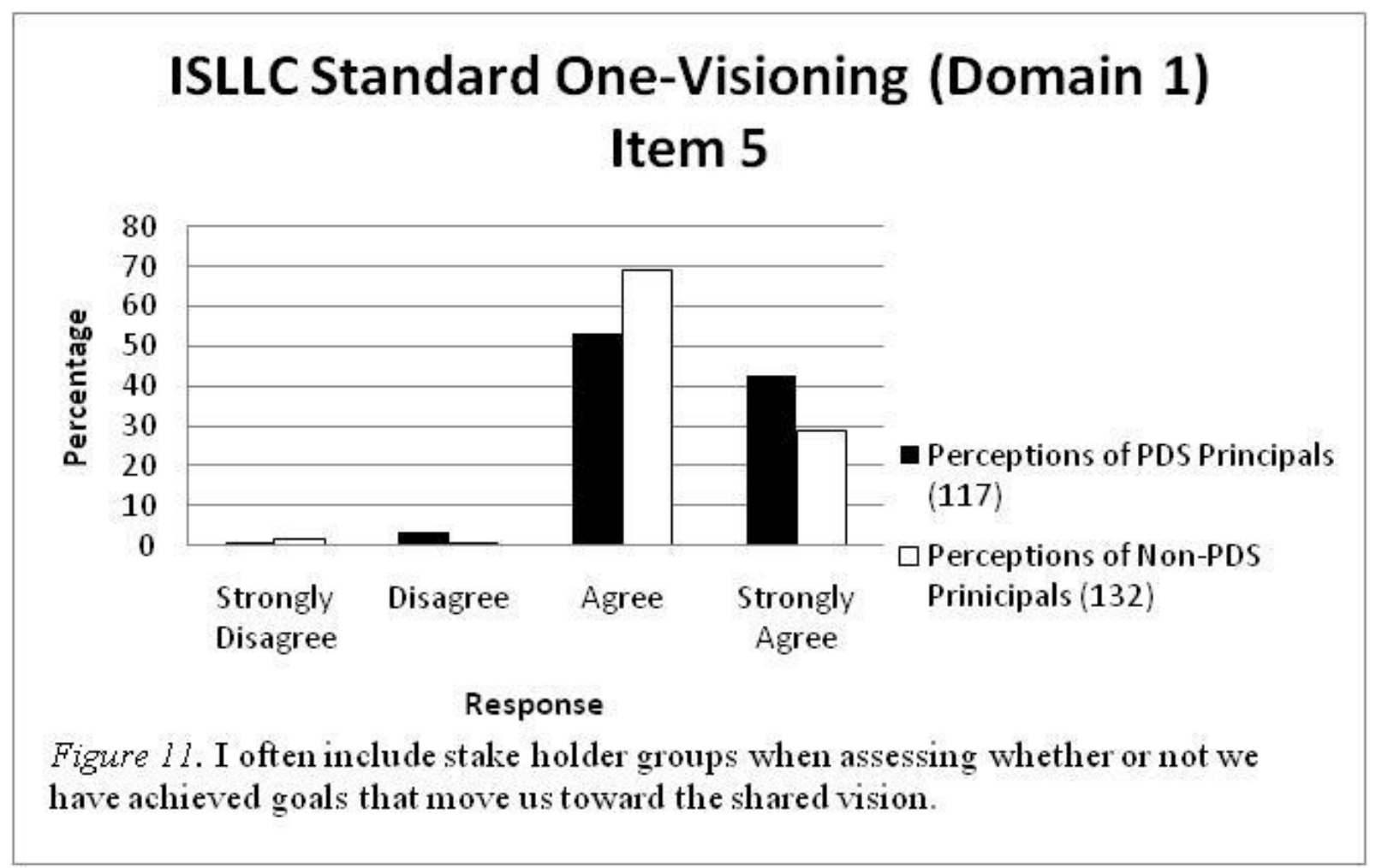

\section{Survey Domain 2 (advocating).}

Advocating: Item 1. My faculty and I have developed and maintained high expectations for all students.

The analysis of the respondents' scores for survey Item 1 in Domain 2 showed that there was not a statistical difference between the responses of the principals who were involved as 
leaders in non-Professional Development Schools compared to principals as leaders in Professional Development Schools. The explanation of this analysis follows.

The comparison group $\mathrm{N}$ was 132 with a mean score of 3.52 and a standard deviation of 0.559. The treatment group $\mathrm{N}$ was 117 with a mean score of 3.61 and a standard deviation of 0.541. [The $t$-Test showed a two-tailed $t$-score of $1.311 \quad p>.05$ (non-significant).] The analysis failed to reject the null hypothesis. There is no statistically significant difference between the scores of the comparison group and the treatment group in Domain 1: Item 6.

The effect size, $d$ calculation, is -0.16 , indicating a small effect of the difference made by the treatment. The negative $d$ indicates that the treatment group scores were larger than the comparison group (see Figure 12).

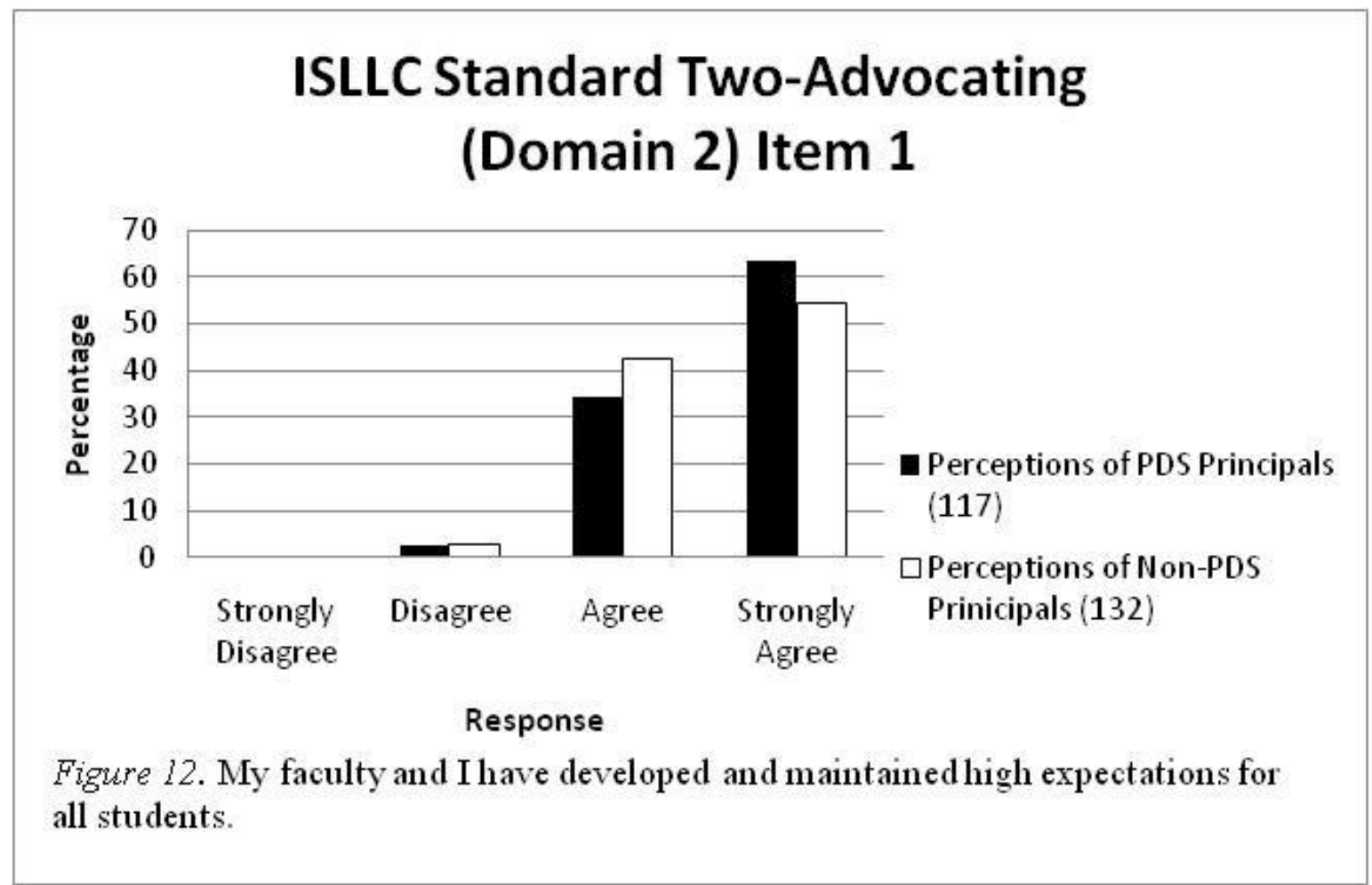

Advocating: Item 2. A rigorous curricular program has been collaboratively developed and is reviewed annually. 
The analysis of the respondents' scores for survey Item 2 in Domain 2 showed there was a statistical difference between the responses of the principals who were involved as leaders in non-Professional Development Schools compared to principals as leaders in Professional Development Schools. The scores of the principals in the treatment group (PDS principals) perceived their schools to be higher performing than the non-PDS principals. The explanation of this analysis follows.

The comparison group $\mathrm{N}$ was 132 with a mean score of 3.30 and a standard deviation of 0.589. The treatment group $\mathrm{N}$ was 117 with a mean score of 3.46 and a standard deviation of 0.550. [The $t$-Test showed a two-tailed $t$-score of $2.29 p>.05$ (non-significant)]. The analysis rejected the null hypothesis. There is a significant difference in the scores of the comparison and treatment groups in Item 2.

The effect size, $d$ calculation, is -0.28 , indicating an effect of the difference made by the treatment. The negative $d$ indicates that the treatment group scores were larger than the comparison group (see Figure 13). 


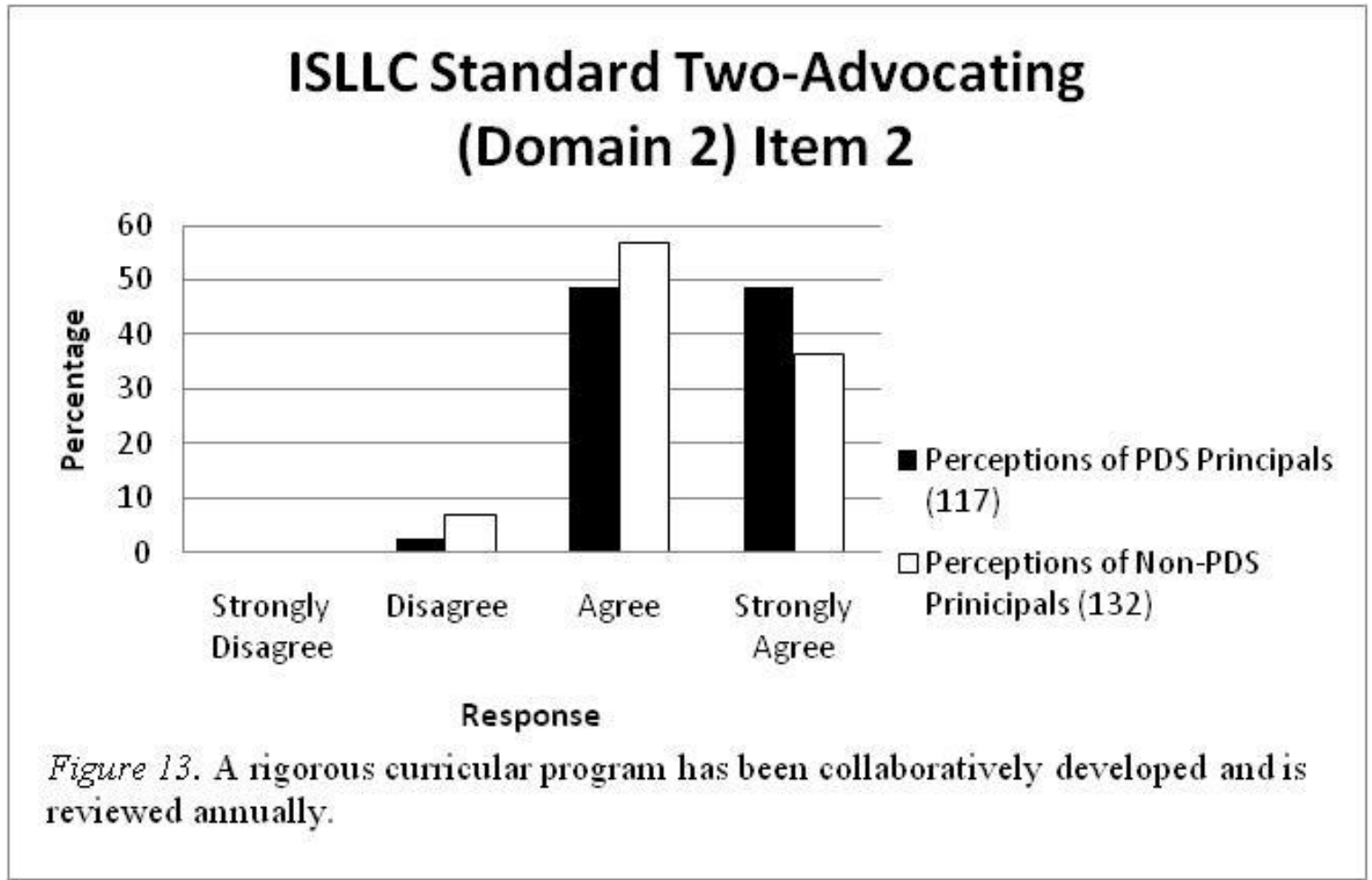

Advocating: Item 3. I believe we have a structured motivational learning environment at this school.

The analysis of the respondents' scores for survey Item 3 in Domain 2 showed that there was not a statistical difference between the responses of the principals who were involved as leaders in non-Professional Development Schools compared to principals as leaders in Professional Development Schools. The explanation of this analysis follows.

The comparison group $\mathrm{N}$ was 132 with a mean score of 3.40 and a standard deviation of 0.578. The treatment group $\mathrm{N}$ was 117 with a mean score of 3.49 and a standard deviation of 0.567. [The $t$-Test showed a two-tailed $t$-score of $1.178 p>.05$ (non-significant).] The analysis failed to reject the null hypothesis. 
The effect size, $d$ calculation, is -0.16 , indicating a small effect of the difference made by the treatment. The negative $d$ indicates that the treatment group scores were larger than the comparison group (see Figure 14).

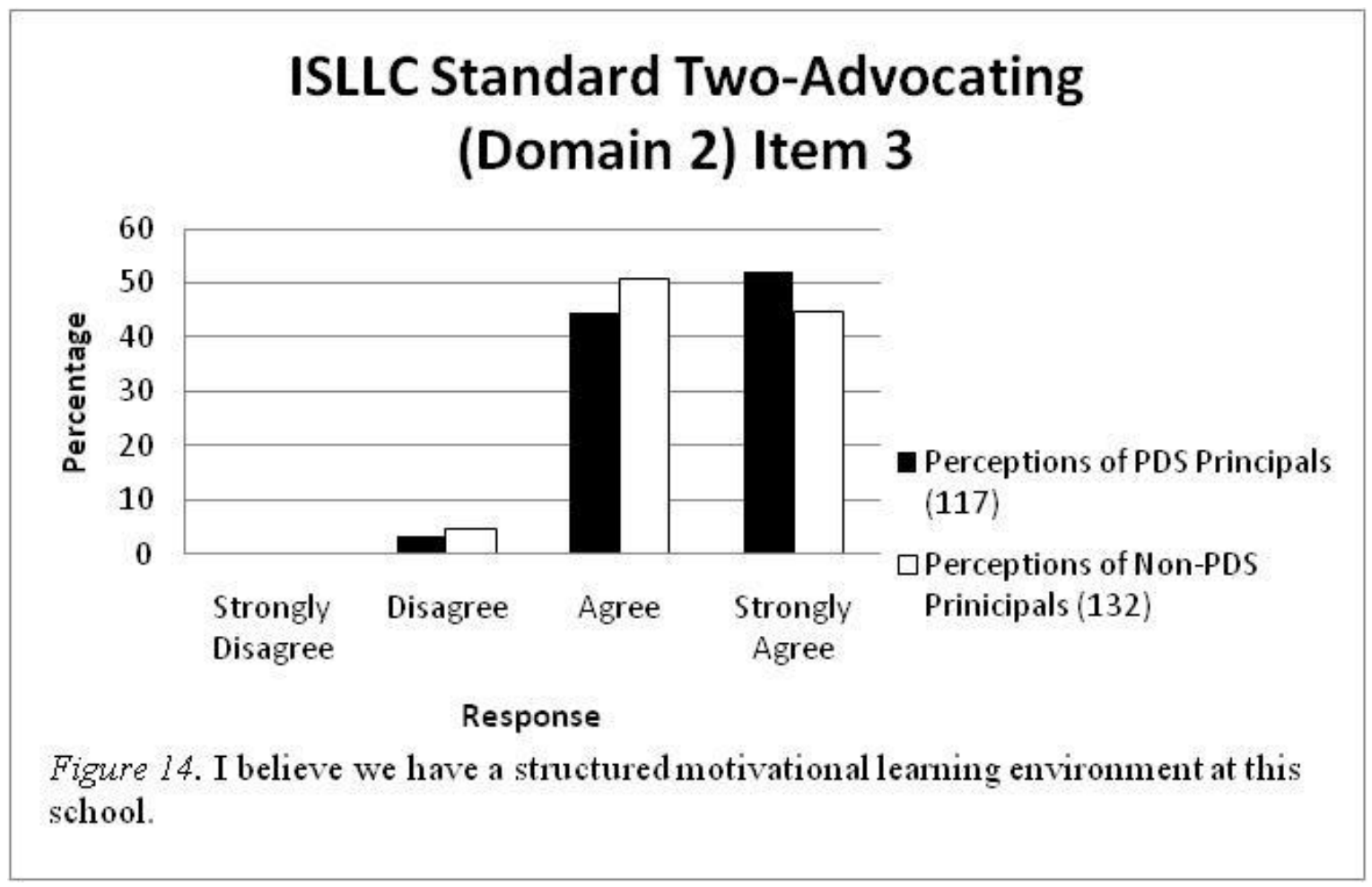

Advocating: Item 4. I have a plan for supervising instruction and I follow it.

The analysis of the respondents' scores for survey Item 4 in Domain 2 showed there was not a statistical difference between the responses of the principals who were involved as leaders in non-Professional Development Schools compared to principals as leaders in Professional Development Schools. The explanation of this analysis follows.

The comparison group $\mathrm{N}$ was 132 with a mean score of 3.32 and a standard deviation of 0.543. The treatment group $\mathrm{N}$ was 117 with a mean score of 3.33 and a standard deviation of 0.525. T [The $t$-Test showed a two-tailed $t$-score of $0.223 \mathrm{p}>.05$ (non-significant).] The analysis 
failed to reject the null hypothesis. There is no statistically significant difference between the scores of the comparison group and the treatment group in Domain 2: Item 4.

The effect size, $d$ calculation, is -0.02 , indicating a small effect of the difference made by the treatment. The negative $d$ indicates that the treatment group scores were larger than the comparison group (see Figure 15).

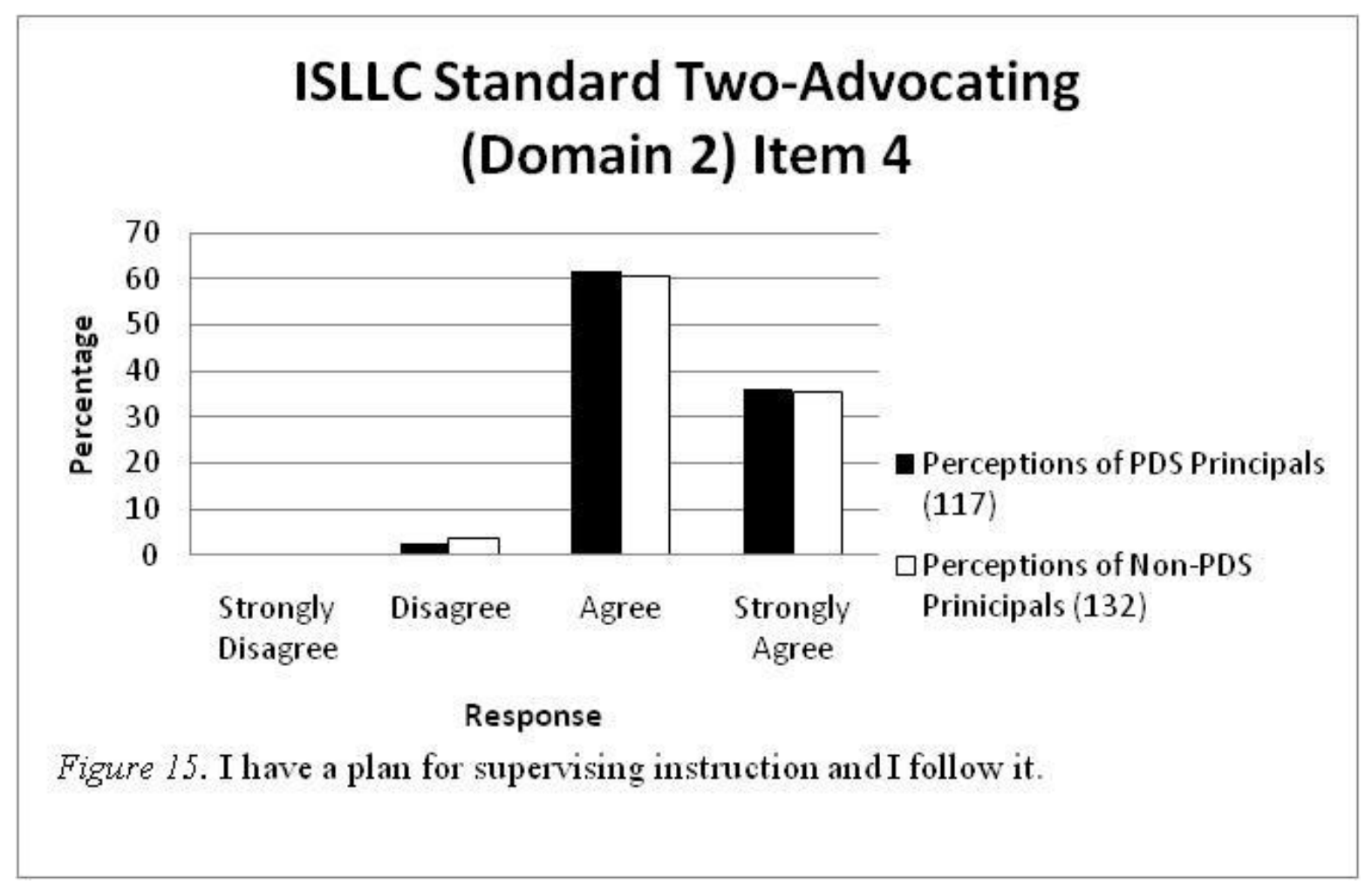

Advocating: Item 5. I do not believe our current assessment system is structured for student growth.

The analysis of the respondents' scores for survey Item 5 in Domain 2 showed there was not a statistical difference between the responses of the principals who were involved as leaders in non-Professional Development Schools compared to principals as leaders in Professional Development Schools. The explanation of this analysis follows. 
The comparison group $\mathrm{N}$ was 132 with a mean score of 2.71 and a standard deviation of 0.921. The treatment group $\mathrm{N}$ was 117 with a mean score of 2.69 and a standard deviation of 0.933. [The $t$-Test showed a two-tailed $t$-score of $-0.168 p>.05$ (non-significant).] The analysis failed to reject the null hypothesis.

There is no statistically significant difference between the scores of the comparison group and the treatment group in Domain 2: Item 5. The effect size, $d$ calculation, is 0.01 , indicating a small effect of the difference made by the treatment (see Figure 16).

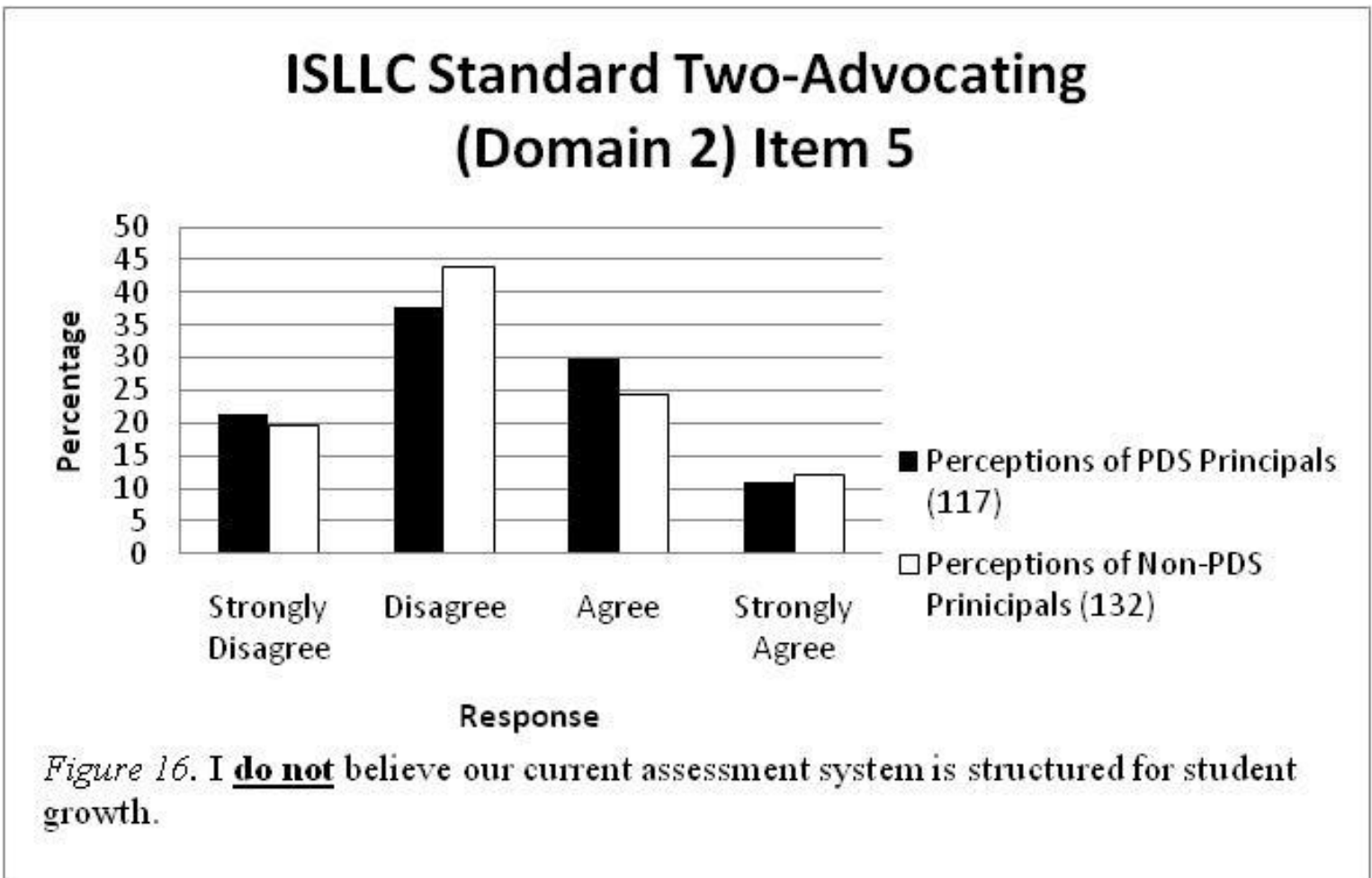

Advocating: Item 6. I believe it is important to develop the instructional capacity of staff.

The analysis of the respondents' scores for survey Item 6 in Domain 2 showed that there was no statistical difference between the responses of the principals who were involved as 
leaders in non-Professional Development Schools compared to principals as leaders in Professional Development Schools. The explanation of this analysis follows.

The comparison group $\mathrm{N}$ was 132 with a mean score of 3.73 and a standard deviation of 0.443. The treatment group $\mathrm{N}$ was 117 with a mean score of 3.71 and a standard deviation of 0.456. [The $t$-Test showed a two-tailed $t$-score of $-0.446 p>.05$ (non-significant).] The analysis failed to reject the null hypothesis. There is no statistically significant difference between the scores of the comparison group and the treatment group in Domain 2: Item 6.

The effect size, $d$ calculation, is 0.04 , indicating a small effect of the difference made by the treatment. The positive $d$ indicates that the comparison group scores were larger than the treatment group 1 (see Figure 17).

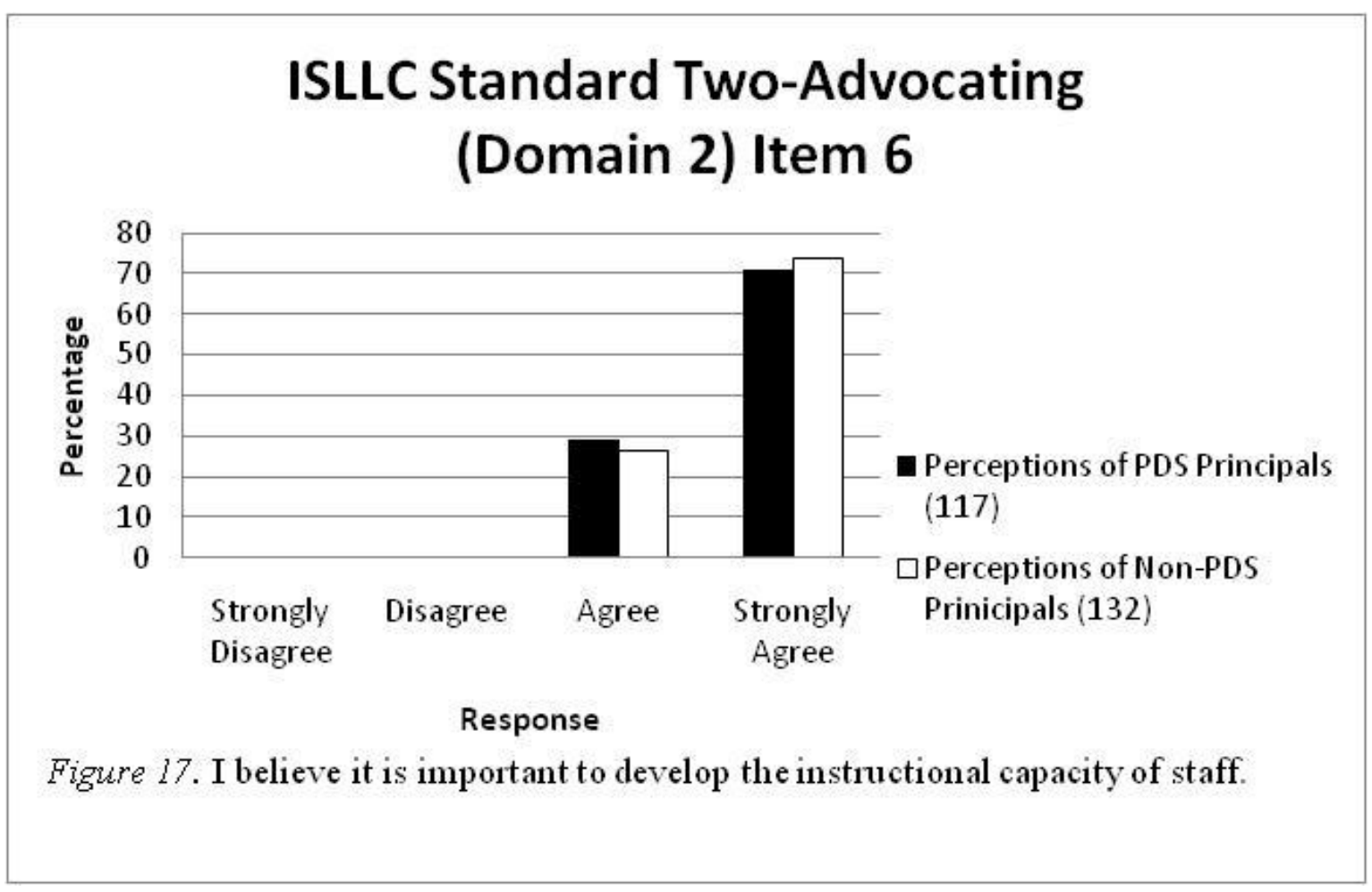

Advocating: Item 7. My faculty, staff, and I value and protect instructional time. 
The analysis of the respondents' scores for survey Item 7 in Domain 2 showed there was not a statistical difference between the responses of the principals who were involved as leaders in non-Professional Development Schools compared to principals as leaders in Professional Development Schools. The explanation of this analysis follows.

The comparison group $\mathrm{N}$ was 132 with a mean score of 3.61 and a standard deviation of 0.519. The treatment group $\mathrm{N}$ was 117 with a mean score of 3.71 and a standard deviation of 0.475. [The $t$-Test showed a two-tailed $t$-score of $1.513 p>.05$ (non-significant).] The analysis failed to reject the null hypothesis. There is no statistically significant difference between the scores of the comparison group and the treatment group in Domain 2: Item 7.

The effect size, $d$ calculation, is -0.20 , indicating a small effect of the difference made by the treatment. The negative $d$ indicates that the treatment group scores were larger than the comparison group (see Figure 18).

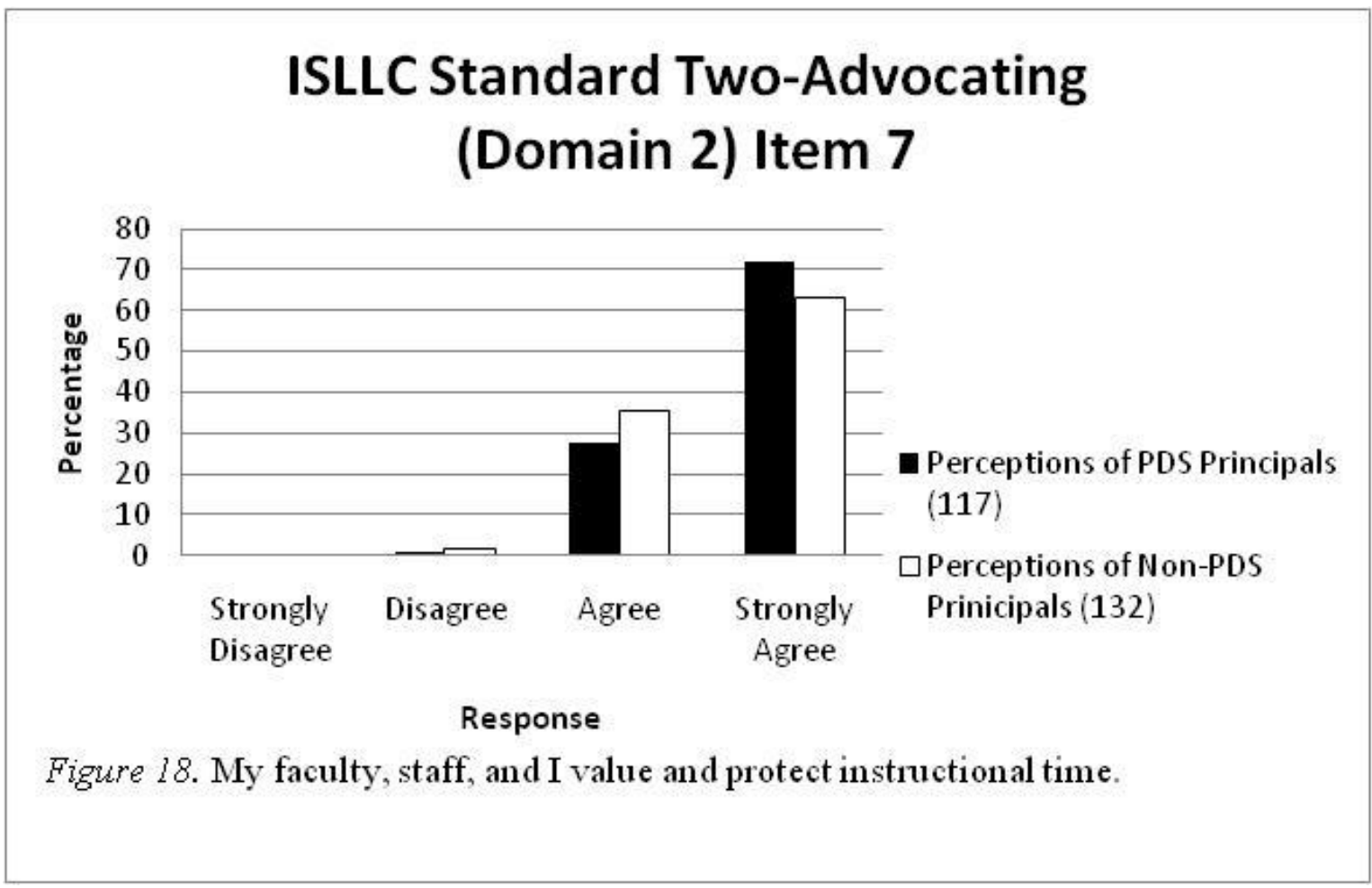


Advocating Item 8. We are constantly engaged in discussions about the most effective instructional technologies, and we seek ways to support getting and using it.

The analysis of the respondents' scores for survey Item 8 in Domain 2 showed there was not a statistical difference between the responses of the principals who were involved as leaders in non-Professional Development Schools compared to principals as leaders in Professional Development Schools. The explanation of this analysis follows.

The comparison group $\mathrm{N}$ was 132 with a mean score of 3.23 and a standard deviation of 0.578. The treatment group $\mathrm{N}$ was 117 with a mean score of 3.27 and a standard deviation of 0.593. [The $t$-Test showed a two-tailed $t$-score of $0.406 p>.05$ (non-significant).] The analysis failed to reject the null hypothesis. There is no statistically significant difference between the scores of the comparison group and the treatment group in Domain 2: Item 8.

The effect size, $d$ calculation, is -0.07 , indicating a small effect of the difference made by the treatment. The negative $d$ indicates that the treatment group scores were larger than the comparison group (see Figure 19). 


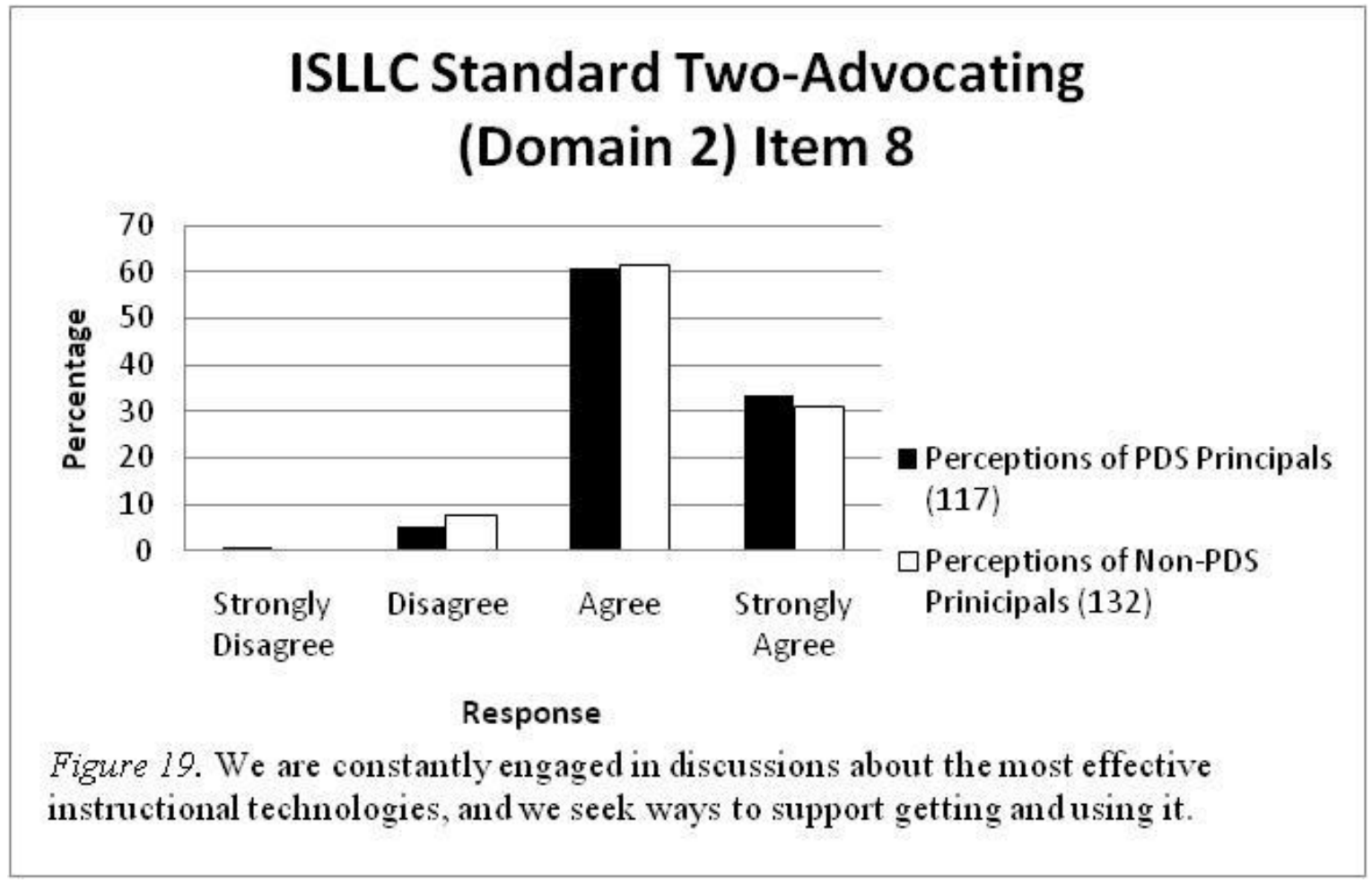

Advocating: Item 9. Evaluation of instructional program impact is done continuously and the program is modified daily to meet students' needs.

The analysis of the respondents' scores for survey Item 9 in Domain 2 showed there was not a statistical difference between the responses of the principals who were involved as leaders in non-Professional Development Schools compared to principals as leaders in Professional Development Schools. The explanation of this analysis follows.

The comparison group $\mathrm{N}$ was 132 with a mean score of 3.02 and a standard deviation of 0.566. The treatment group $\mathrm{N}$ was 117 with a mean score of 3.08 and a standard deviation of 0.589. [The $t$-Test showed a two-tailed $t$-score of $0.843 p>.05$ (non-significant).] The analysis failed to reject the null hypothesis. There is no statistically significant difference between the scores of the comparison group and the treatment group in Domain 2: Item 9. 
The effect size, $d$ calculation, is -0.10 , indicating a small effect of the difference made by the treatment. The negative $d$ indicates that the treatment group scores were larger than the comparison group (see Figure 20).

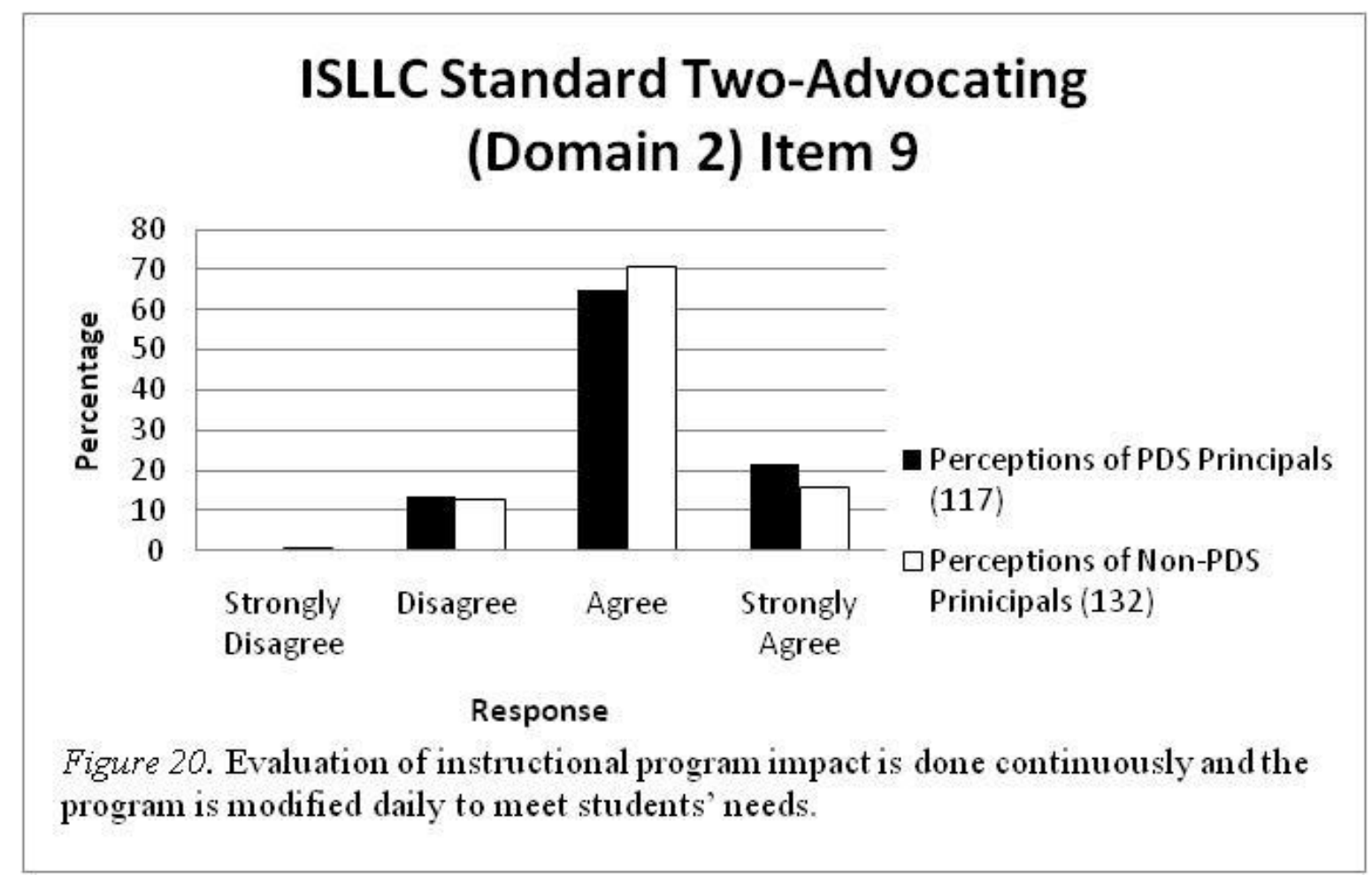

\section{Survey Domain 3 (management)}

Management: Item 1. I constantly review operational systems to determine if they are meeting the needs of all stakeholders.

The analysis of the respondents' scores for survey Item 1 in Domain 3 showed there was not a statistical difference between the responses of the principals who were involved as leaders in non-Professional Development Schools compared to principals as leaders in Professional Development Schools. The explanation of this analysis follows.

The comparison group $\mathrm{N}$ was 132 with a mean score of 3.12 and a standard deviation of 0.479. The treatment group $\mathrm{N}$ was 117 with a mean score of 3.16 and a standard deviation of 
0.556. [The $t$-Test showed a two-tailed $t$-score of $0.628 p>.05$ (non-significant).] The analysis failed to reject the null hypothesis. There is no statistically significant difference between the scores of the comparison group and the treatment group in Domain 3: Item 1.

The effect size, $d$ calculation, is -0.08 , indicating a small effect of the difference made by the treatment. The negative $d$ indicates that the treatment group scores were larger than the comparison group (see Figure 21).

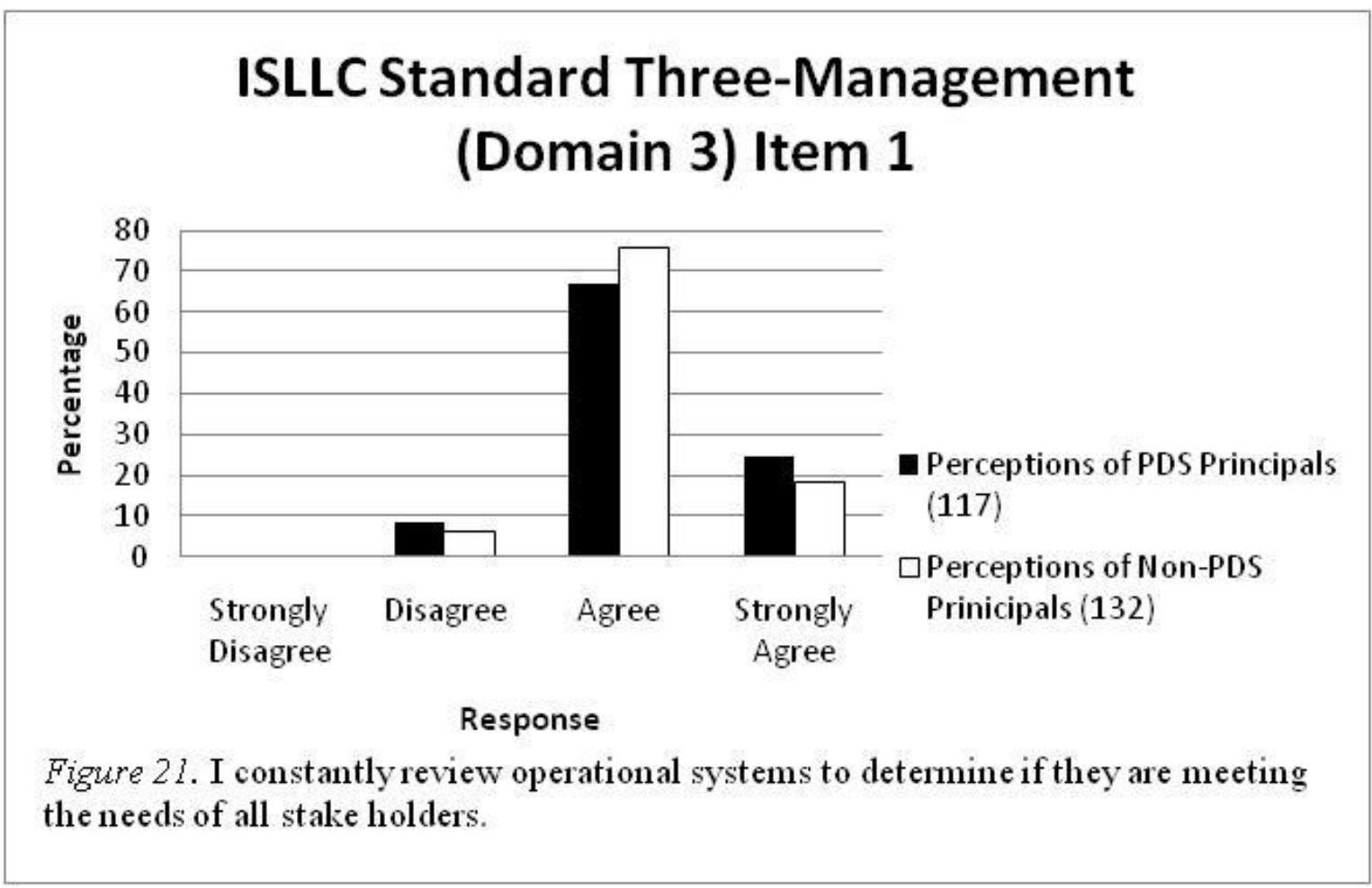

Management: Item 2. I challenge my faculty and staff to find ways to maximize resources.

The analysis of the respondents' scores for survey Item 2 in Domain 3 showed there was not a statistical difference between the responses of the principals who were involved as leaders in non-Professional Development Schools compared to principals as leaders in Professional Development Schools. The explanation of this analysis follows. 
The comparison group $\mathrm{N}$ was 132 with a mean score of 3.41 and a standard deviation of 0.524. The treatment group $\mathrm{N}$ was 117 with a mean score of 3.46 and a standard deviation of 0.518. [The $t$-Test showed a two-tailed $t$-score of $0.793 p>.05$ (non-significant).] The analysis failed to reject the null hypothesis. There is no statistically significant difference between the scores of the comparison group and the treatment group in Domain 3: Item 2.

The effect size, $d$ calculation, is -0.10 , indicating a small effect of the difference made by the treatment. The negative $d$ indicates that the treatment group scores were larger than the comparison group (see Figure 22).

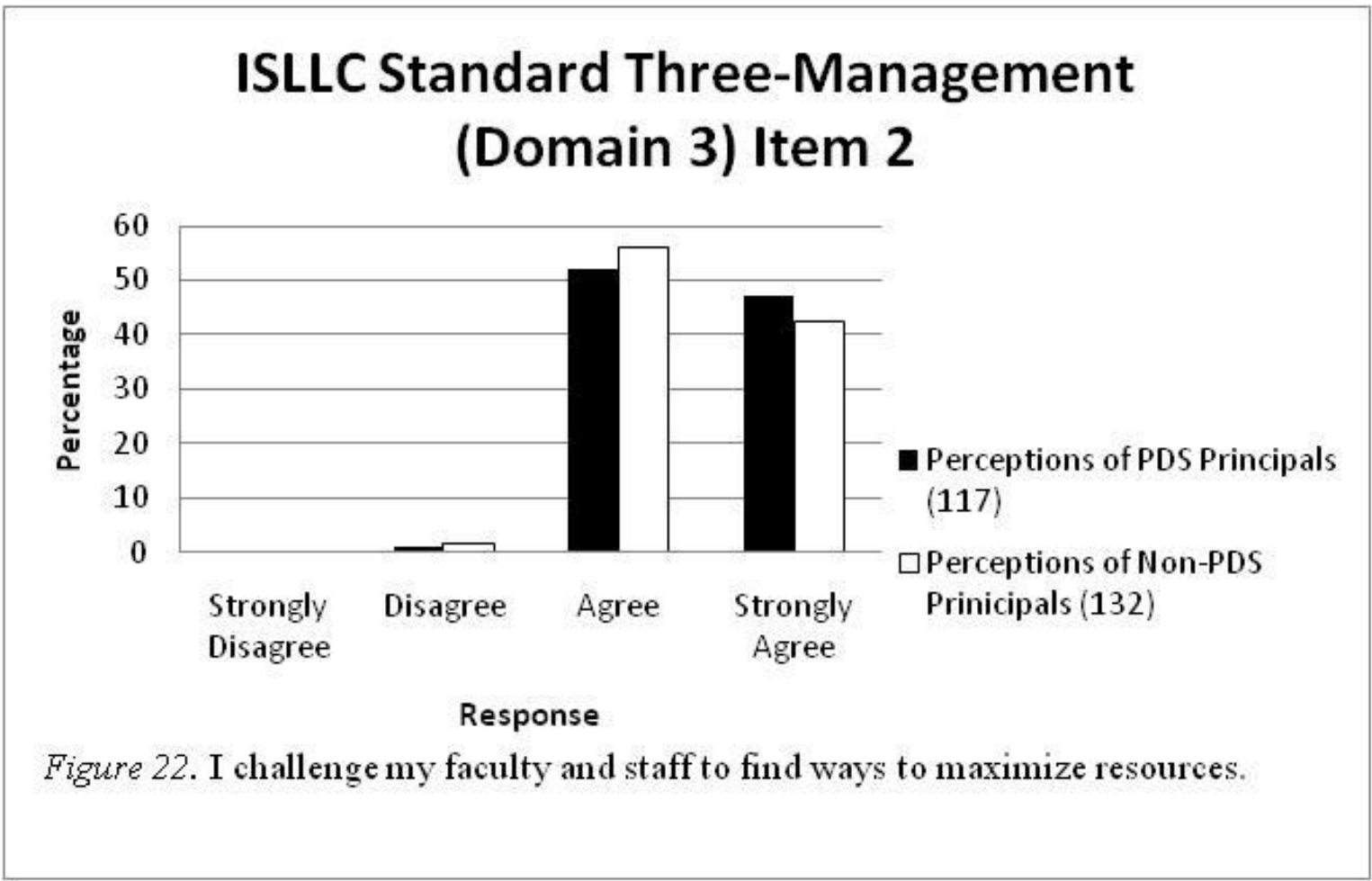

Management :Item 3. Safety policy and procedures are at the forefront of continuous review.

The analysis of the respondents' scores for survey Item 3 in Domain 3 showed there was not a statistical difference between the responses of the principals who were involved as leaders 
in non-Professional Development Schools compared to principals as leaders in Professional Development Schools. The explanation of this analysis follows.

The comparison group $\mathrm{N}$ was 132 with a mean score of 3.52 and a standard deviation of 0.572. The treatment group $\mathrm{N}$ was 117 with a mean score of 3.56 and a standard deviation of 0.532. [The $t$-Test showed a two-tailed $t$-score of $0.467 p>.05$ (non-significant).] The analysis failed to reject the null hypothesis. There is no statistically significant difference between the scores of the comparison group and the treatment group in Domain 3: Item 3.

The effect size, $d$ calculation, is -0.07 , indicating a small effect of the difference made by the treatment. The negative $d$ indicates that the treatment group scores were larger than the comparison group (see Figure 23).

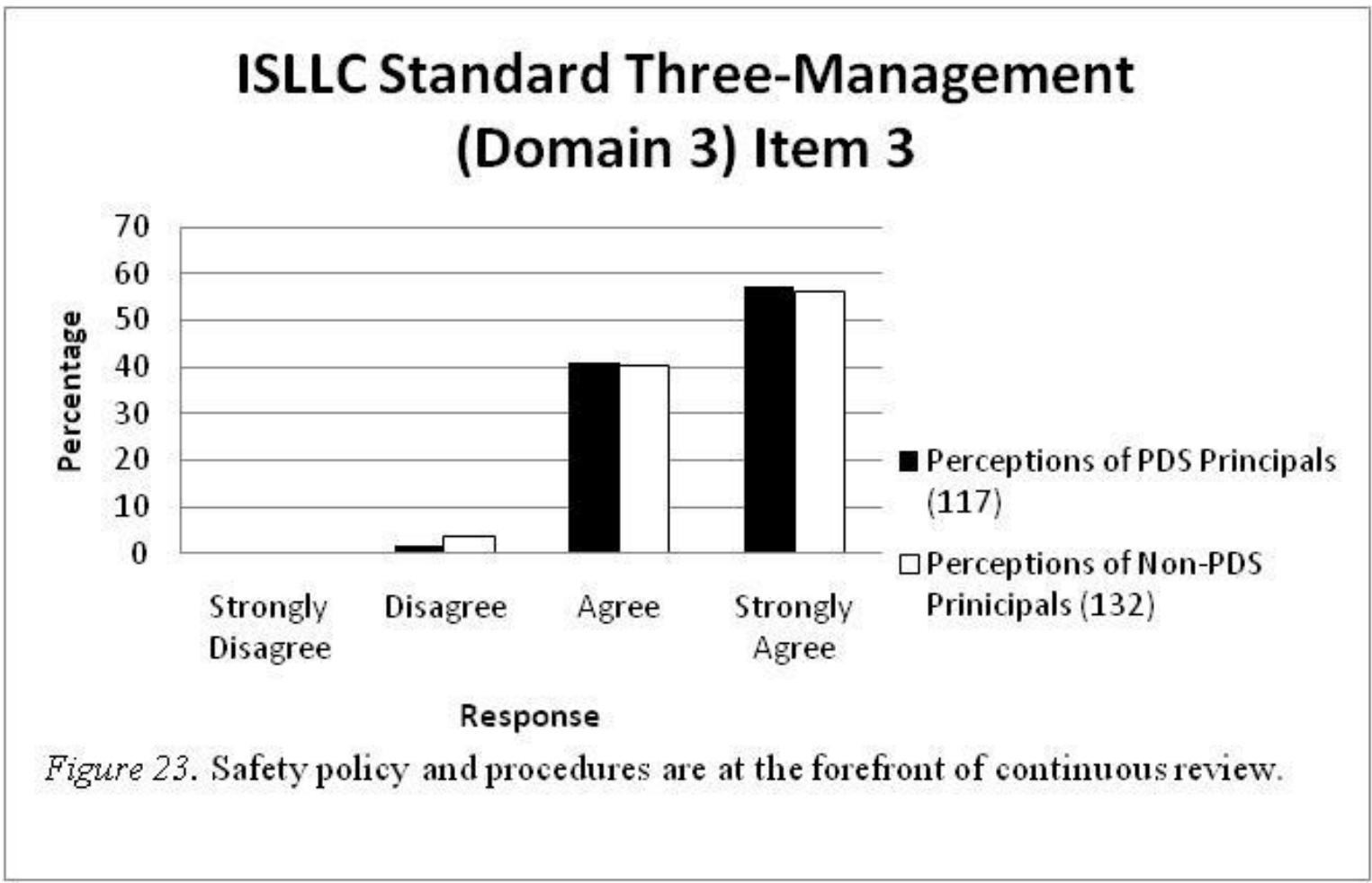


Management: Item 4. I develop leadership capacity in my faculty and staff by enabling them to solve organizational problems.

The analysis of the respondents' scores for survey Item 4 in Domain 3 showed there was not a statistical difference between the responses of the principals who were involved as leaders in non-Professional Development Schools compared to principals as leaders in Professional Development Schools. The explanation of this analysis follows.

The comparison group $\mathrm{N}$ was 132 with a mean score of 3.47 and a standard deviation of 0.545. The treatment group $\mathrm{N}$ was 117 with a mean score of 3.48 and a standard deviation of 0.519. [The $t$-Test showed a two-tailed $t$-score of $0.132 p>.05$ (non-significant).] The analysis failed to reject the null hypothesis. There is no statistically significant difference between the scores of the comparison group and the treatment group in Domain 3: Item 4.

The effect size, $d$ calculation, is -0.02 , indicating a small effect of the difference made by the treatment. The negative $d$ indicates that the treatment group scores were larger than the comparison group (see Figure 24). 


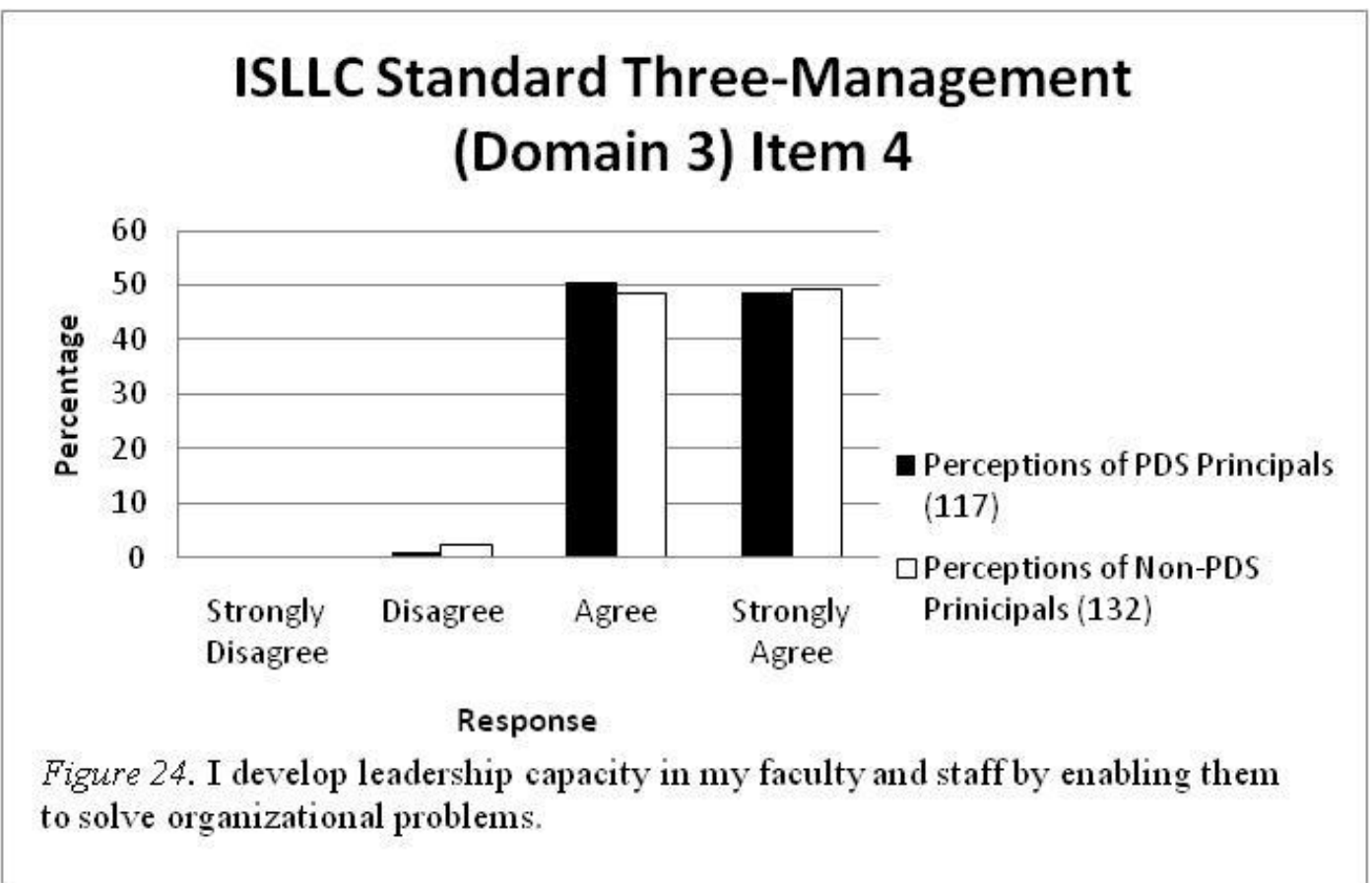

Management: Item 5. Focused teaching and learning time is built into logistics.

The analysis of the respondents' scores for survey Item 5 in Domain 3 showed there was not a statistical difference between the responses of the principals who were involved as leaders in non-Professional Development Schools compared to principals as leaders in Professional Development Schools. The explanation of this analysis follows.

The comparison group $\mathrm{N}$ was 132 with a mean score of 3.39 and a standard deviation of 0.575. The treatment group $\mathrm{N}$ was 117 with a mean score of 3.46 and a standard deviation of 0.498. [The $t$-Test showed a two-tailed $t$-score of $0.722 p>.05$ (non-significant).] The analysis failed to reject the null hypothesis. There is no statistically significant difference between the scores of the comparison group and the treatment group in Domain 3: Item 5.

The effect size, $d$ calculation, is -0.13 , indicating a small effect of the difference made by the treatment. The negative $d$ indicates that the treatment group scores were larger than the comparison group (see Figure 25). 


\section{ISLLC Standard Three-Management (Domain 3) Item 5}

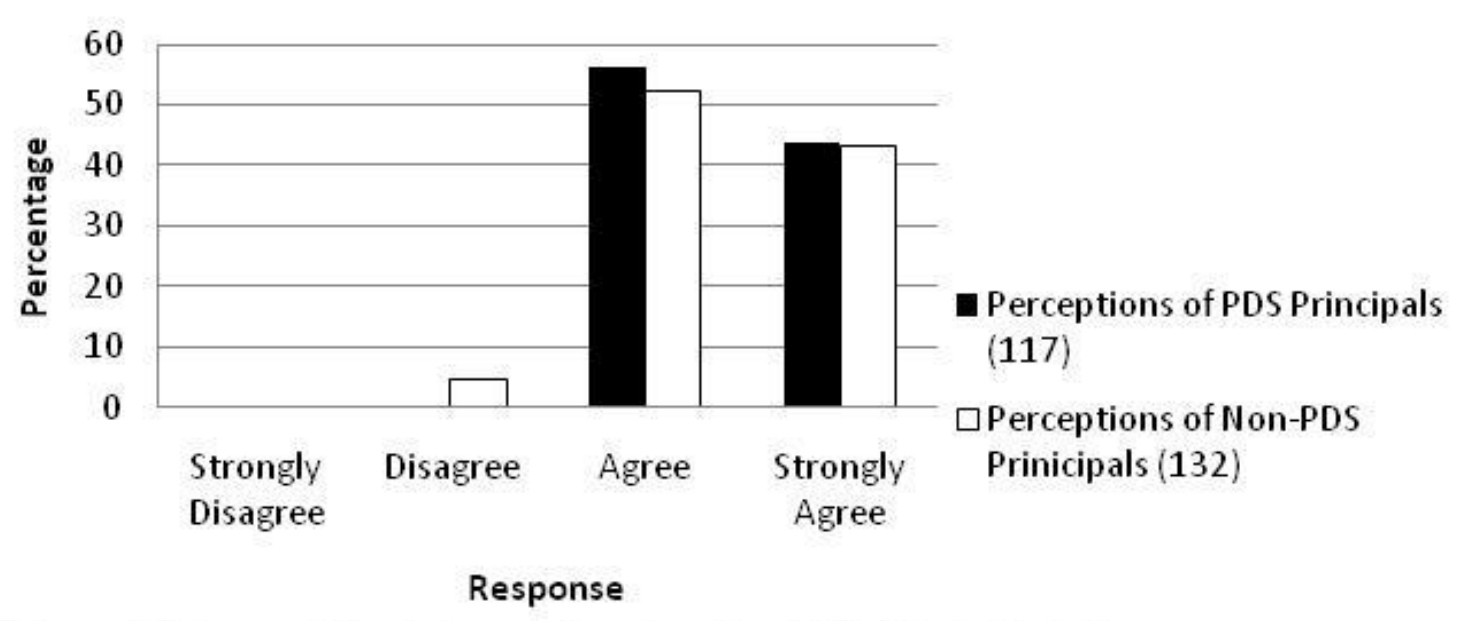

Figure 25. Focused teaching and learning time is built into logistics.

Survey Domain 4 (collaboration). Collaboration: Item 1. The faculty and I analyze data pertinent to the educational environment. The analysis of the respondents' scores for survey Item 1 in Domain 4 showed there was not a statistical difference between the responses of the principals who were involved as leaders in non-Professional Development Schools compared to principals as leaders in Professional Development Schools. The explanation of this analysis follows.

The comparison group $\mathrm{N}$ was 132 with a mean score of 3.57 and a standard deviation of 0.497. The treatment group $\mathrm{N}$ was 117 with a mean score of 3.52 and a standard deviation of 0.519. [The $t$-Test showed a two-tailed $t$-score of $-0.727 p>.05$ (non-significant).] The analysis failed to reject the null hypothesis. There is no statistically significant difference between the scores of the comparison group and the treatment group in Domain 4: Item 1. 


\section{ISLLC Standard Four-Collaboration (Domain 4) Item 1}

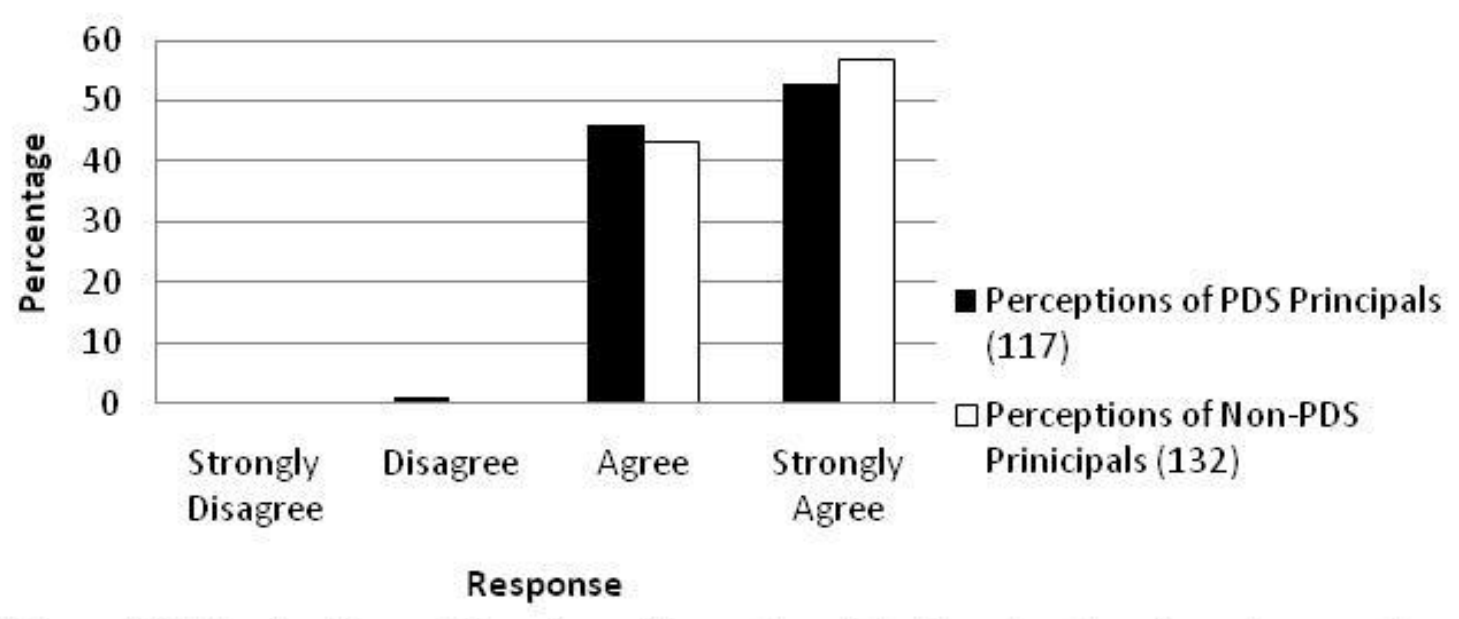

Figure 26 . The faculty and I analyze data pertinent to the educational environment.

The effect size, $d$ calculation, is 0.10 , indicating a small effect of the difference made by the treatment. The positive $d$ indicates that the comparison group scores were larger than the treatment group (see Figure 26).

Collaboration: Item 2 . I expect faculty and staff members to welcome and engage the community.

The analysis of the respondents' scores for survey Item 2 in Domain 4 showed there was not a statistical difference between the responses of the principals who were involved as leaders in non-Professional Development Schools compared to principals as leaders in Professional Development Schools. The explanation of this analysis follows.

The comparison group $\mathrm{N}$ was 132 with a mean score of 3.63 and a standard deviation of 0.500. The treatment group $\mathrm{N}$ was 117 with a mean score of 3.69 and a standard deviation of 0.464. [The $t$-Test showed a two-tailed $t$-score of $1.035 p>.05$ (non-significant).] The analysis 
failed to reject the null hypothesis. There is no statistically significant difference between the scores of the comparison group and the treatment group in Domain 4: Item 2.

The effect size, $d$ calculation, is -0.12 , indicating a small effect of the difference made by the treatment. The negative $d$ indicates that the treatment group scores were larger than the comparison group (see Figure 27).

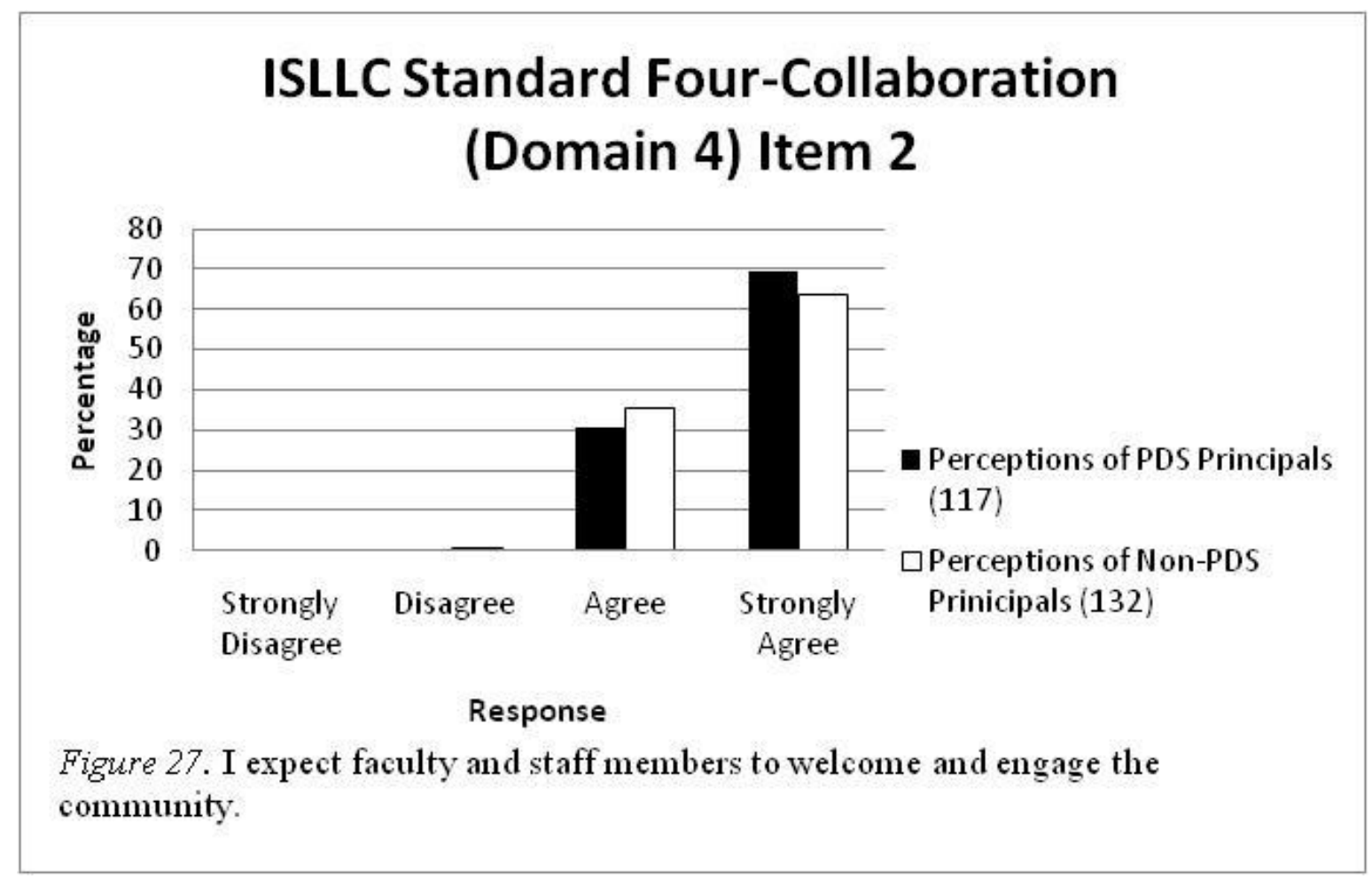

Collaboration: Item 3. The well-being of students and their families give us a common purpose for building positive relationships.

The analysis of the respondents' scores for survey Item 3 in Domain 4 showed there was not a statistical difference between the responses of the principals who were involved as leaders in non-Professional Development Schools compared to principals as leaders in Professional Development Schools. The explanation of this analysis follows. 
The comparison group $\mathrm{N}$ was 132 with a mean score of 3.67 and a standard deviation of 0.489. The treatment group $\mathrm{N}$ was 117 with a mean score of 3.61 and a standard deviation of 0.508. [The $t$-Test showed a two-tailed $t$-score of $-0.946 \quad p>.05$ (non-significant).] The analysis failed to reject the null hypothesis. There is no statistically significant difference between the scores of the comparison group and the treatment group in Domain 4: Item 3.

The effect size, $d$ calculation, is 0.12 , indicating a small effect of the difference made by the treatment. The positive $d$ indicates that the comparison group scores were larger than the treatment group (see Figure 28).

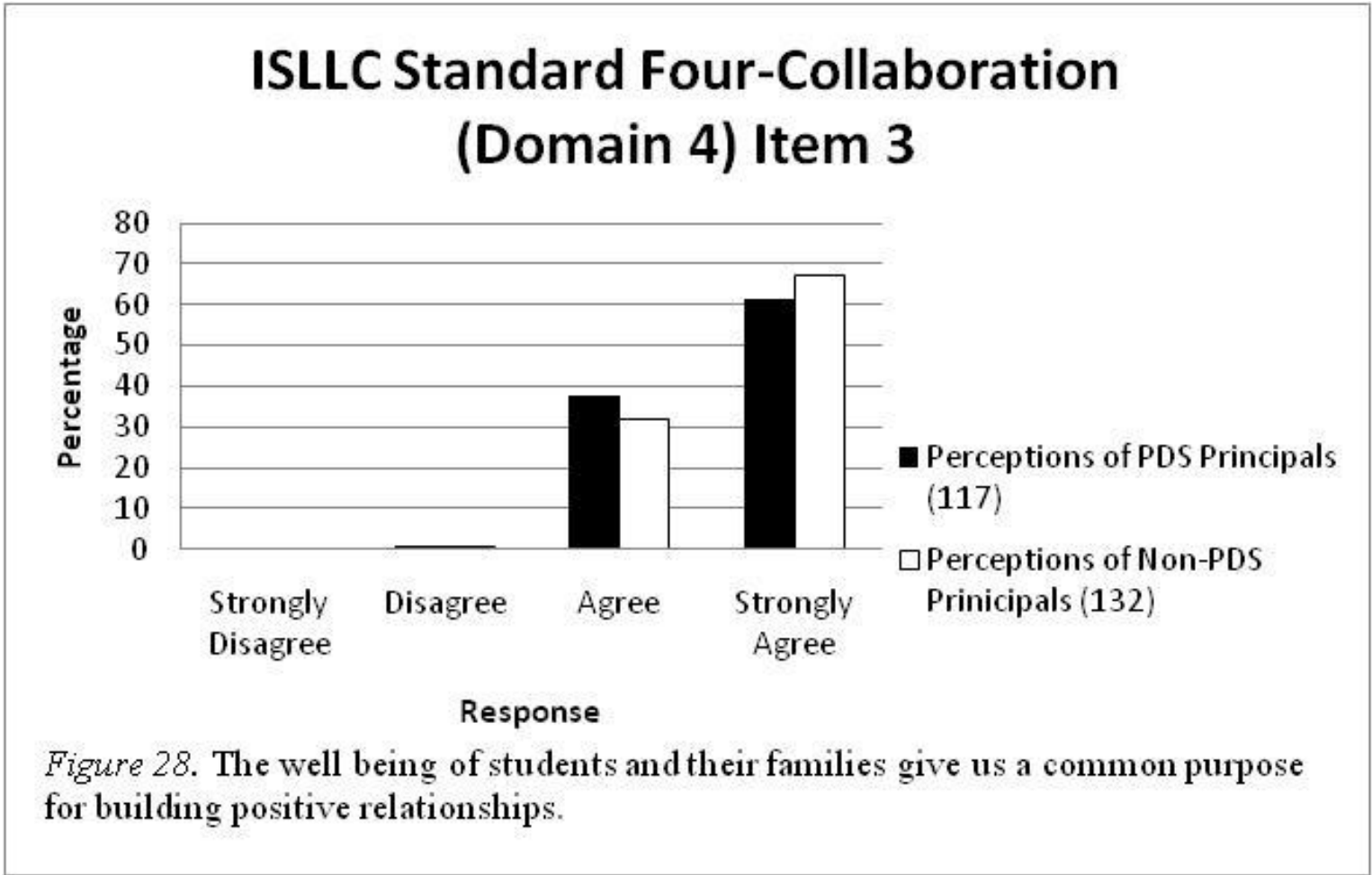

Collaboration: Item 4. I don't waste my time reaching out to disinterested community partners.

The analysis of the respondents' scores for survey Item 4 in Domain 4 showed there was not a statistical difference between the responses of the principals who were involved as leaders 
in non-Professional Development Schools compared to principals as leaders in Professional Development Schools. The explanation of this analysis follows.

The comparison group $\mathrm{N}$ was 132 with a mean score of 2.89 and a standard deviation of 0.645. The treatment group $\mathrm{N}$ was 117 with a mean score of 2.91 and a standard deviation of 0.707. [The $t$-Test showed a two-tailed $t$-score of $0.141 \quad p>.05$ (non-significant).] The analysis failed to reject the null hypothesis. There is no statistically significant difference between the scores of the comparison group and the treatment group in Domain 4: Item 4.

The effect size, $d$ calculation, is -0.03 , indicating a small effect of the difference made by the treatment. The negative $d$ indicates that the treatment group scores were larger than the comparison group (see Figure 29).

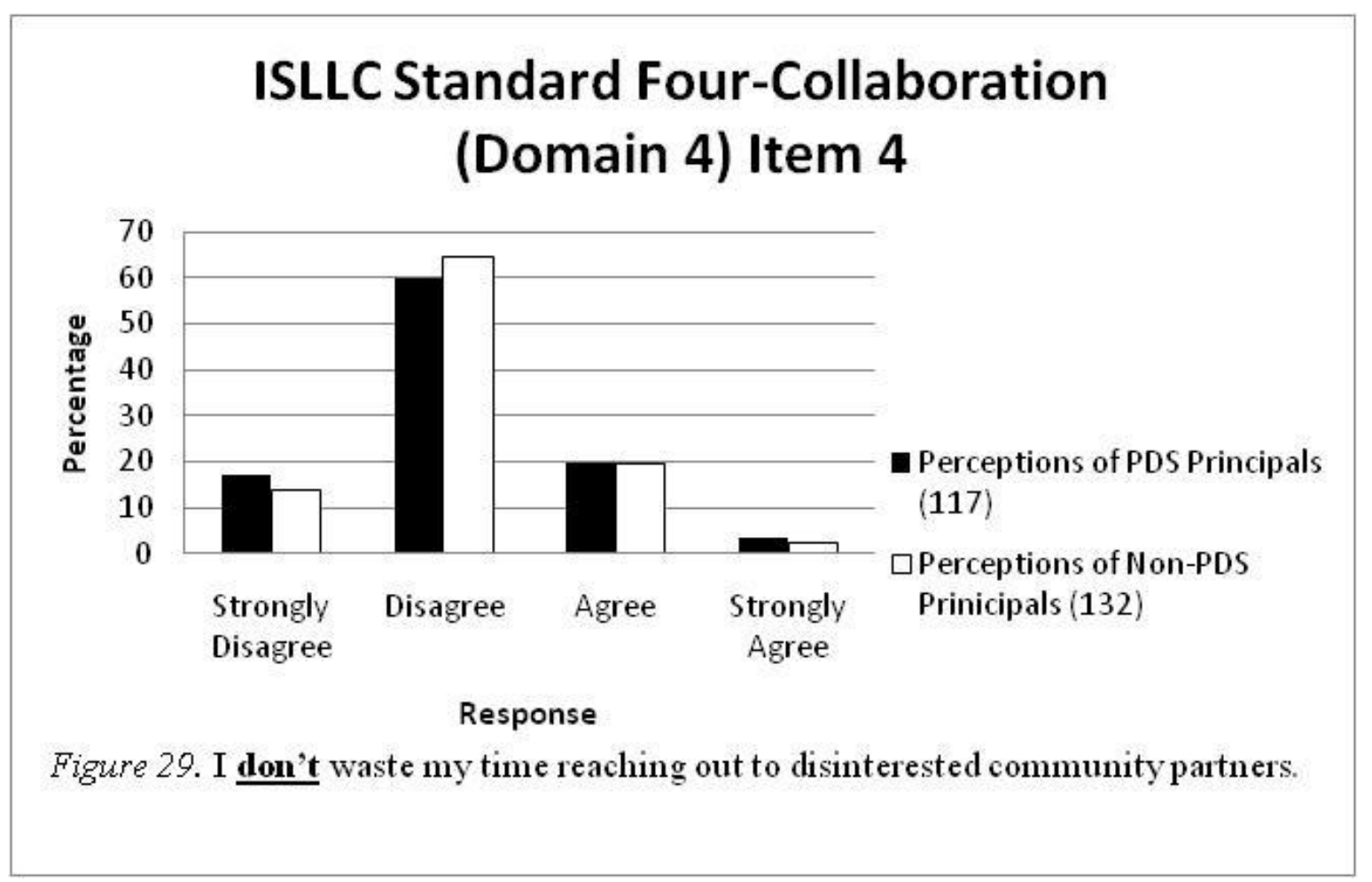




\section{Survey Domain 5 (ethics): Item 1}

Ethics: Item 1. I do not think it is important that every student maximize his/her achievement or success.

The analysis of the respondents' scores for survey Item 1 in Domain 5 showed there was not a statistical difference between the responses of the principals who were involved as leaders in non-Professional Development Schools compared to principals as leaders in Professional Development Schools. The explanation of this analysis follows.

The comparison group $\mathrm{N}$ was 132 with a mean score of 3.80 and a standard deviation of 0.549 . The treatment group $\mathrm{N}$ was 117 with a mean score of 3.67 and a standard deviation of 0.766 . [The $t$-Test showed a two-tailed $t$-score of $-1.538 \quad p>.05$ (non-significant).] The analysis failed to reject the null hypothesis. There is no statistically significant difference between the scores of the comparison group and the treatment group in Domain 5: Item 1

The effect size, $d$ calculation, is 0.20 , indicating a small effect of the difference made by the treatment. The positive $d$ indicates that the comparison group scores were larger than the treatment group (see Figure 30). 


\section{ISLLC Standard Five-Ethics (Domain 5) Item 1}

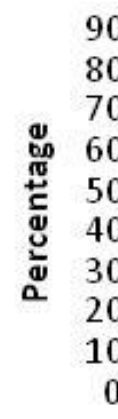

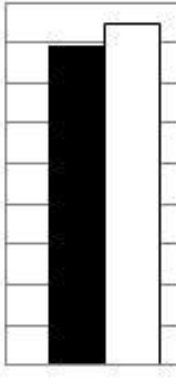

Strongly

Disagree

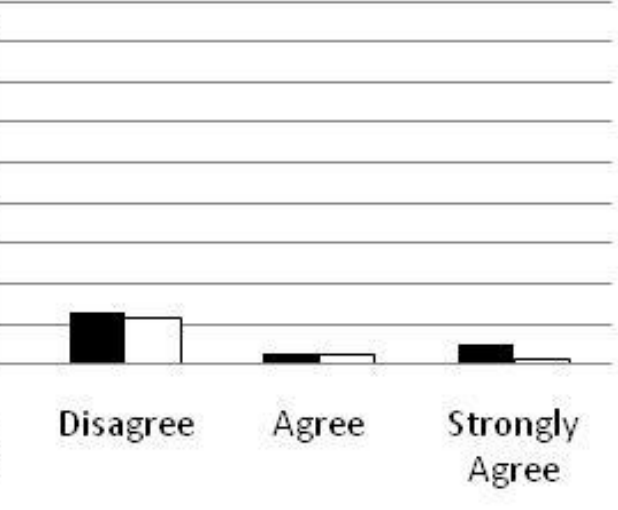

Perceptions of PDS Principals (117)

$\square$ Perceptions of Non-PDS

Response

Prinicipals (132)

Figure 30.1 do not thin
achievement or success.

Ethics: Item 2. I model the kind of behavior I expect from everyone else.

The analysis of the respondents' scores for survey Item 2 in Domain 5 showed there was not a statistical difference between the responses of the principals who were involved as leaders in non-Professional Development Schools compared to principals as leaders in Professional Development Schools. The explanation of this analysis follows.

The comparison group $\mathrm{N}$ was 132 with a mean score of 3.77 and a standard deviation of 0.426. The treatment group $\mathrm{N}$ was 117 with a mean score of 3.71 and a standard deviation of 0.456. [The $t$-Test showed a two-tailed $t$-score of $-0.998 p>.05$ (non-significant).] The analysis failed to reject the null hypothesis. There is no statistically significant difference between the scores of the comparison group and the treatment group in Domain 5: Item 2. 
The effect size, $d$ calculation, is 0.14 , indicating a small effect of the difference made by the treatment. The positive $d$ indicates that the comparison group scores were larger than the treatment group (see Figure 31).

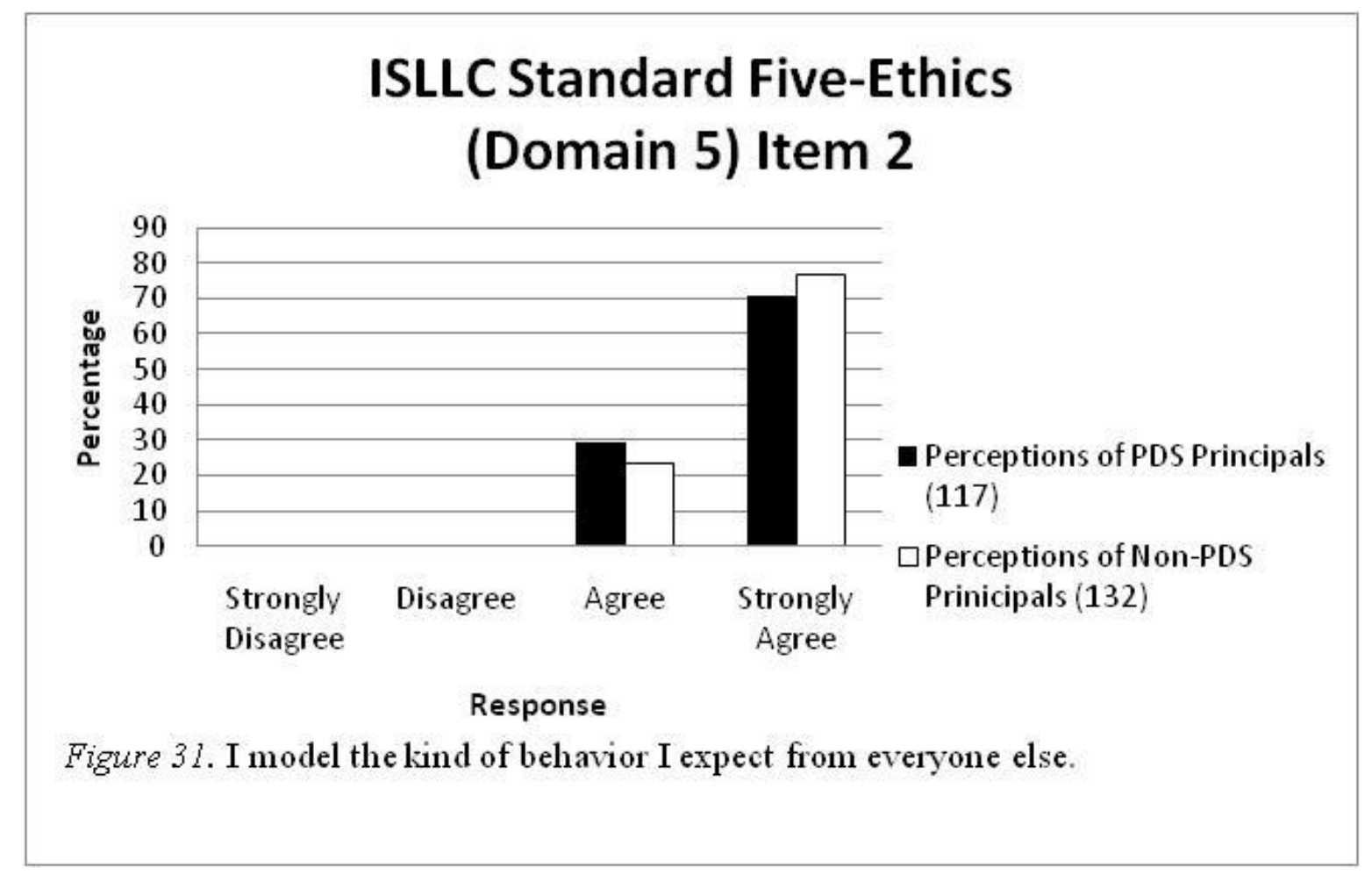

Ethics: Item 3. My vision for building a fear free culture of teaching and learning is centered by democratic principles, equality and fairness, and strength in diversity.

The analysis of the respondents' scores for survey Item 3 in Domain 5 showed there was not a statistical difference between the responses of the principals who were involved as leaders in non-Professional Development Schools compared to principals as leaders in Professional Development Schools. The explanation of this analysis follows. 
The comparison group $\mathrm{N}$ was 132 with a mean score of 3.53 and a standard deviation of 0.581. The treatment group $\mathrm{N}$ was 117 with a mean score of 3.51 and a standard deviation of 0.546. [The $t$-Test showed a two-tailed $t$-score of $0.313 p>.05$ (non-significant).] The analysis failed to reject the null hypothesis. There is no statistically significant difference between the scores of the comparison group and the treatment group in Domain 5: Item 3.

The effect size, $d$ calculation, is 0.04 , indicating a small effect of the difference made by the treatment. The positive $d$ indicates that the comparison group scores were larger than the treatment group (see Figure 32).

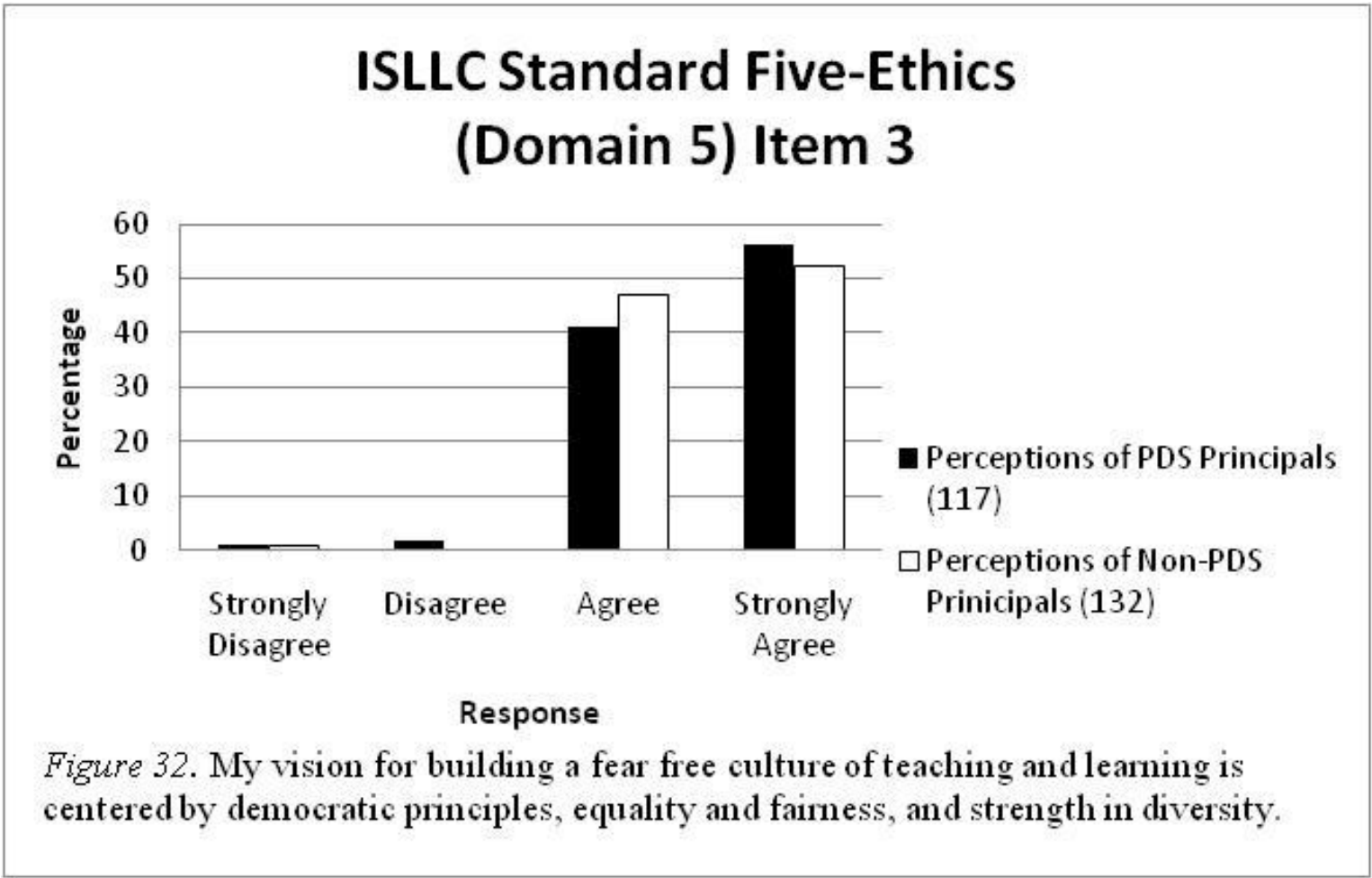

Ethics: Item 4. Legal consequences of decision-making are not connected to moral decision making.

The analysis of the respondents' scores for survey Item 4 in Domain 5 showed there was not a statistical difference between the responses of the principals who were involved as leaders 
in non-Professional Development Schools compared to principals as leaders in Professional Development Schools. The explanation of this analysis follows.

The comparison group $\mathrm{N}$ was 132 with a mean score of 2.43 and a standard deviation of 0.608. The treatment group $\mathrm{N}$ was 117 with a mean score of 2.45 and a standard deviation of 0.609. [The $t$-Test showed a two-tailed $t$-score of $0.274 p>.05$ (non-significant).] The analysis failed to reject the null hypothesis. There is no statistically significant difference between the scores of the comparison group and the treatment group in Domain 5: Item 4.

The effect size, $d$ calculation, is -0.03 , indicating a small effect of the difference made by the treatment. The negative $d$ indicates that the treatment group scores were larger than the comparison group (see Figure 33).

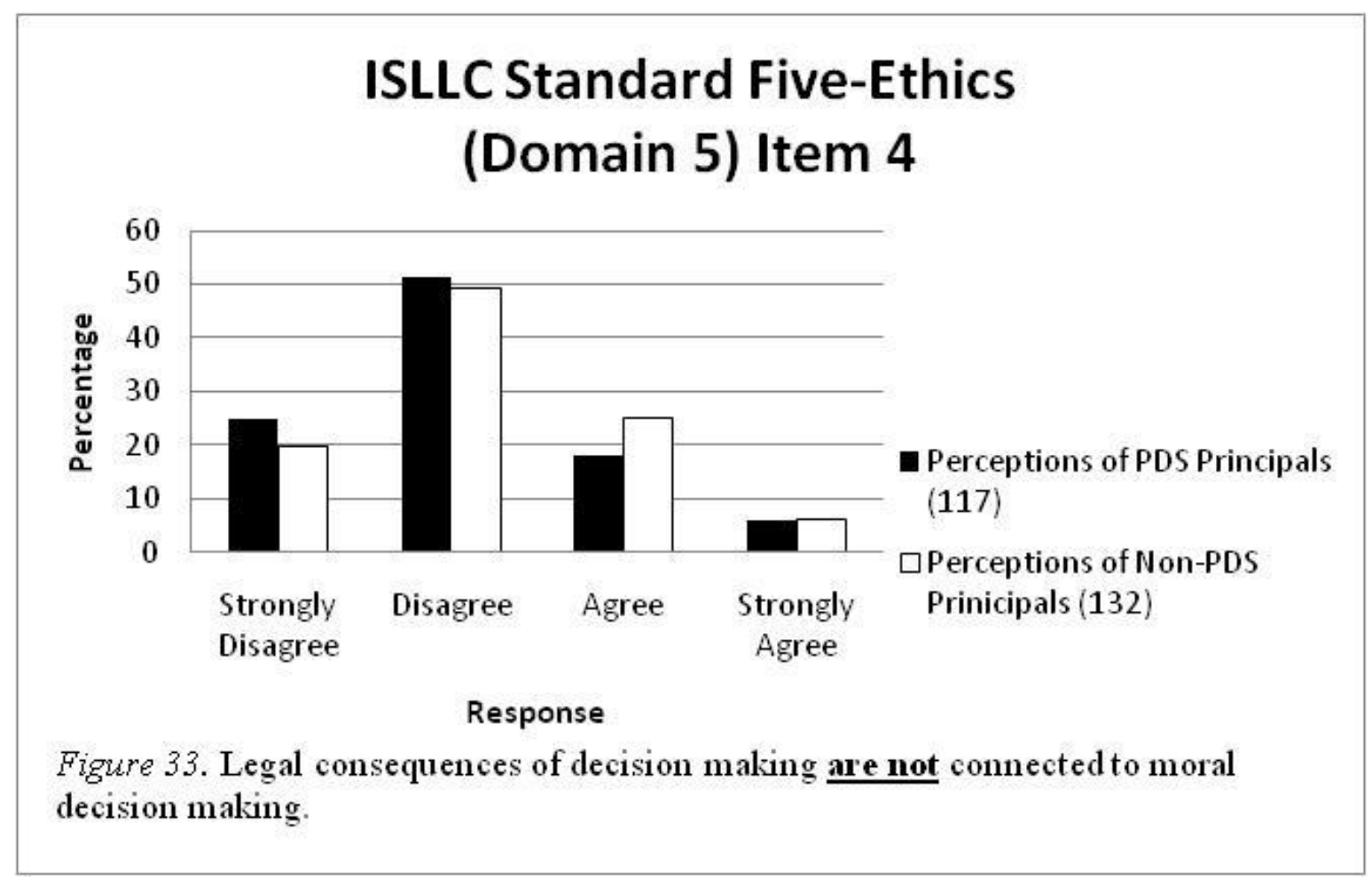


Ethics: Item 5. I base all my leadership work on opening the door of education to all people regardless of social status, ethnicity, gender, religion, and cultural background.

The analysis of the respondents' scores for survey Item 5 in Domain 5 showed there was not a statistical difference between the responses of the principals who were involved as leaders in non-Professional Development Schools compared to principals as leaders in Professional Development Schools. The explanation of this analysis follows.

The comparison group $\mathrm{N}$ was 132 with a mean score of 3.73 and a standard deviation of 0.420. The treatment group $\mathrm{N}$ was 117 with a mean score of 3.80 and a standard deviation of 0.496. [The $t$-Test showed a two-tailed $t$-score of $1.299 \quad p>.05$ (non-significant).] The analysis failed to reject the null hypothesis. There is no statistically significant difference between the scores of the comparison group and the treatment group in Domain 5: Item 5.

The effect size, $d$ calculation, is -0.15 , indicating a small effect of the difference made by the treatment. The negative $d$ indicates that the treatment group scores were larger than the comparison group (see Figure 34). 


\section{ISLLC Standard Five-Ethics (Domain 5) Item 5}

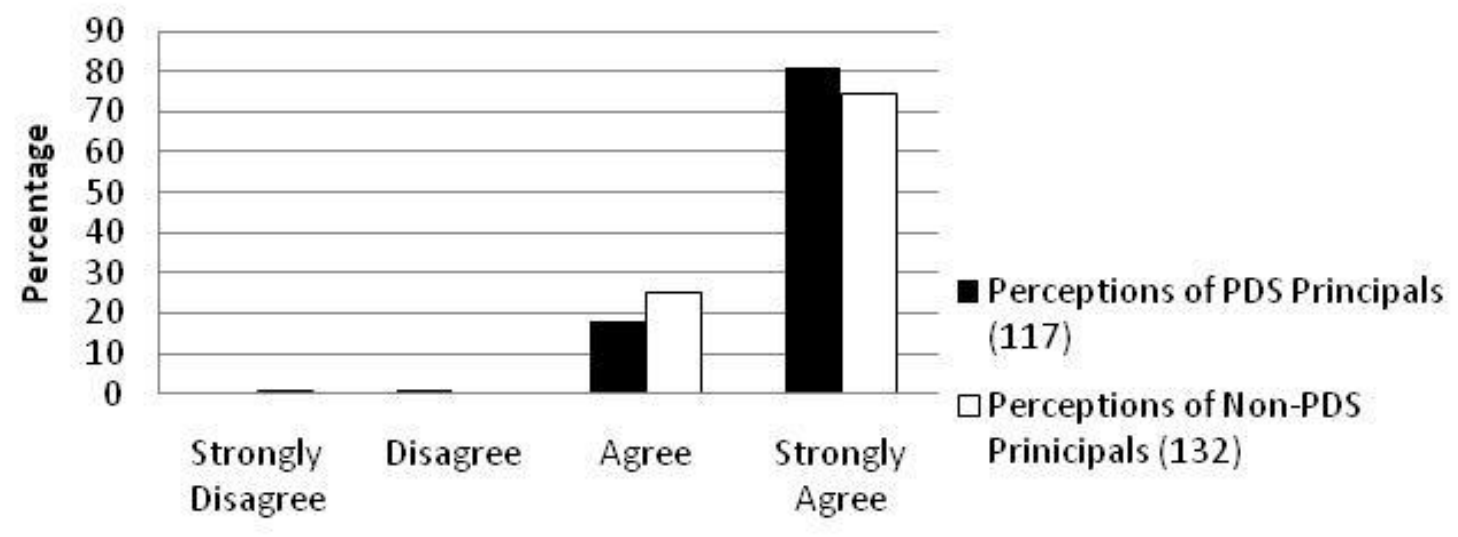

Response

Figure 34. I base all my leadership work on opening the door of education to all people regardless of social status, ethnicity, gender, religion, and cultural background.

\section{Survey Domain 6 (Community Relations): Item 1}

Community relations: Item 1. I and my faculty advocate for children and their families.

The analysis of the respondents' scores for survey Item 1 in Domain 6 showed there was not a statistical difference between the responses of the principals who were involved as leaders in non-Professional Development Schools compared to principals as leaders in Professional Development Schools. The explanation of this analysis follows.

The comparison group $\mathrm{N}$ was 132 with a mean score of 3.62 and a standard deviation of 0.567. The treatment group $\mathrm{N}$ was 117 with a mean score of 3.62 and a standard deviation of 0.523. [The $t$-Test showed a two-tailed $t$-score of $0.397 \quad p>.05$ (non-significant).] The analysis failed to reject the null hypothesis. 
There is no statistically significant difference between the scores of the comparison group and the treatment group in Domain 6: Item 1. The effect size, $d$ calculation, is 0.00 , indicating no effect of the difference made by the treatment (see Figure 35).

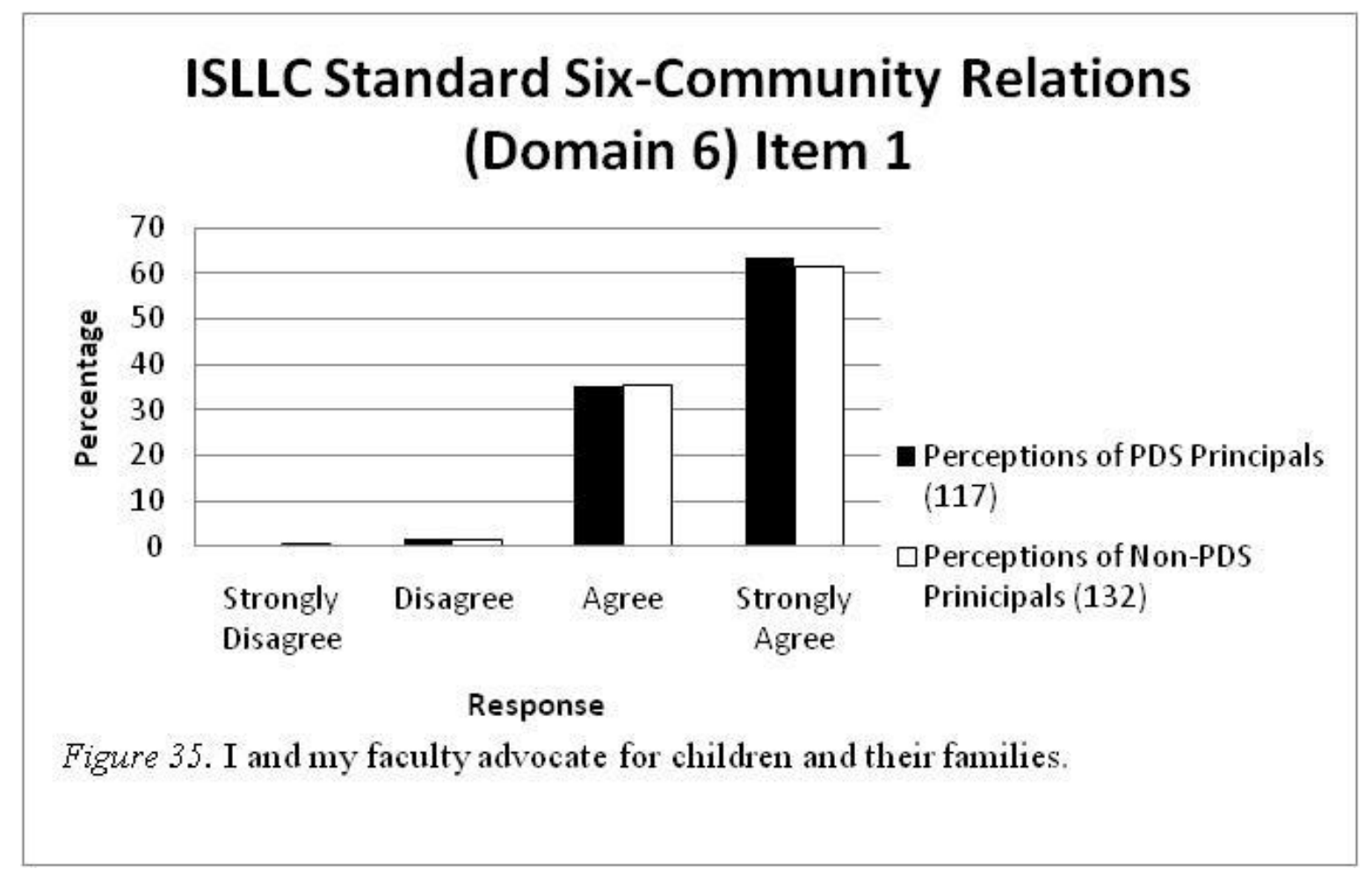

Community relations: Item 2. I act to influence policies affecting student learning.

The analysis of the respondents' scores for survey Item 2 in Domain 6 showed there was not a statistical difference between the responses of the principals who were involved as leaders in non-Professional Development Schools compared to principals as leaders in Professional Development Schools. The explanation of this analysis follows.

The comparison group $\mathrm{N}$ was 132 with a mean score of 3.52 and a standard deviation of 0.586. The treatment group $\mathrm{N}$ was 117 with a mean score of 3.54 and a standard deviation of 0.534. [The $t$-Test showed a two-tailed $t$-score of $0.271 \quad p>.05$ (non-significant).] The analysis 
failed to reject the null hypothesis. There is no statistically significant difference between the scores of the comparison group and the treatment group in Domain 6: Item 2.

The effect size, $d$ calculation, is -0.04 , indicating a small effect of the difference made by the treatment. The negative $d$ indicates that the treatment group scores were larger than the comparison group (see Figure 36).

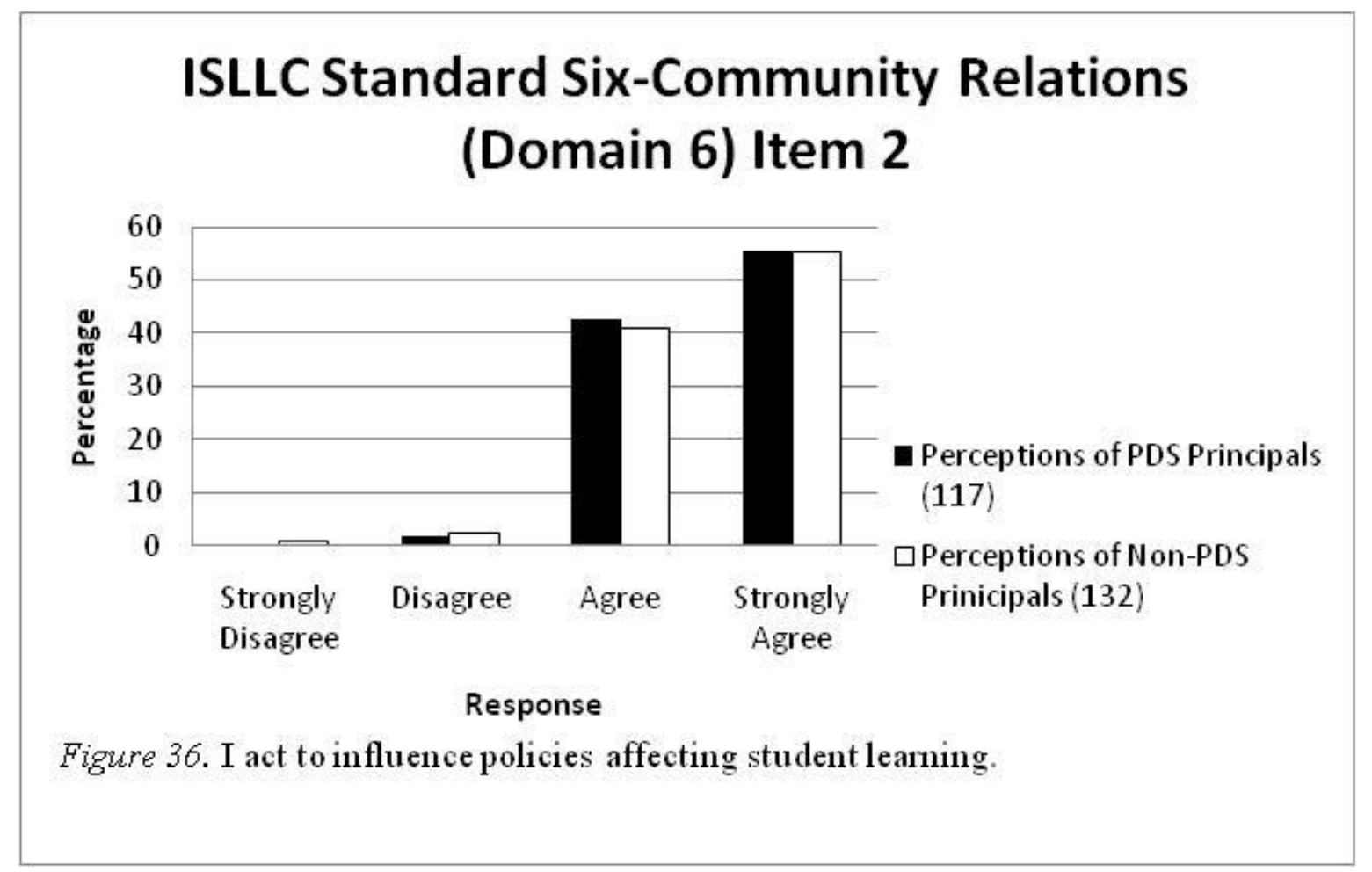

Community Relations: Item 3. I anticipate emerging trends and adapt my leadership strategies.

The analysis of the respondents' scores for survey Item 3 in Domain 6 showed there was not a statistical difference between the responses of the principals who were involved as leaders in non-Professional Development Schools compared to principals as leaders in Professional Development Schools. The explanation of this analysis follows. 
The comparison group $\mathrm{N}$ was 132 with a mean score of 3.35 and a standard deviation of 0.567. The treatment group $\mathrm{N}$ was 117 with a mean score of 3.29 and a standard deviation of 0.558. [The $t$-Test showed a two-tailed $t$-score of -.845 $p>.05$ (non-significant).] The analysis failed to reject the null hypothesis. There is no statistically significant difference between the scores of the comparison group and the treatment group in Domain 6: Item 3.

The effect size, $d$ calculation, is 0.11 , indicating a small effect of the difference made by the treatment. The positive $d$ indicates that the comparison group scores were larger than the treatment group (see Figure 37).

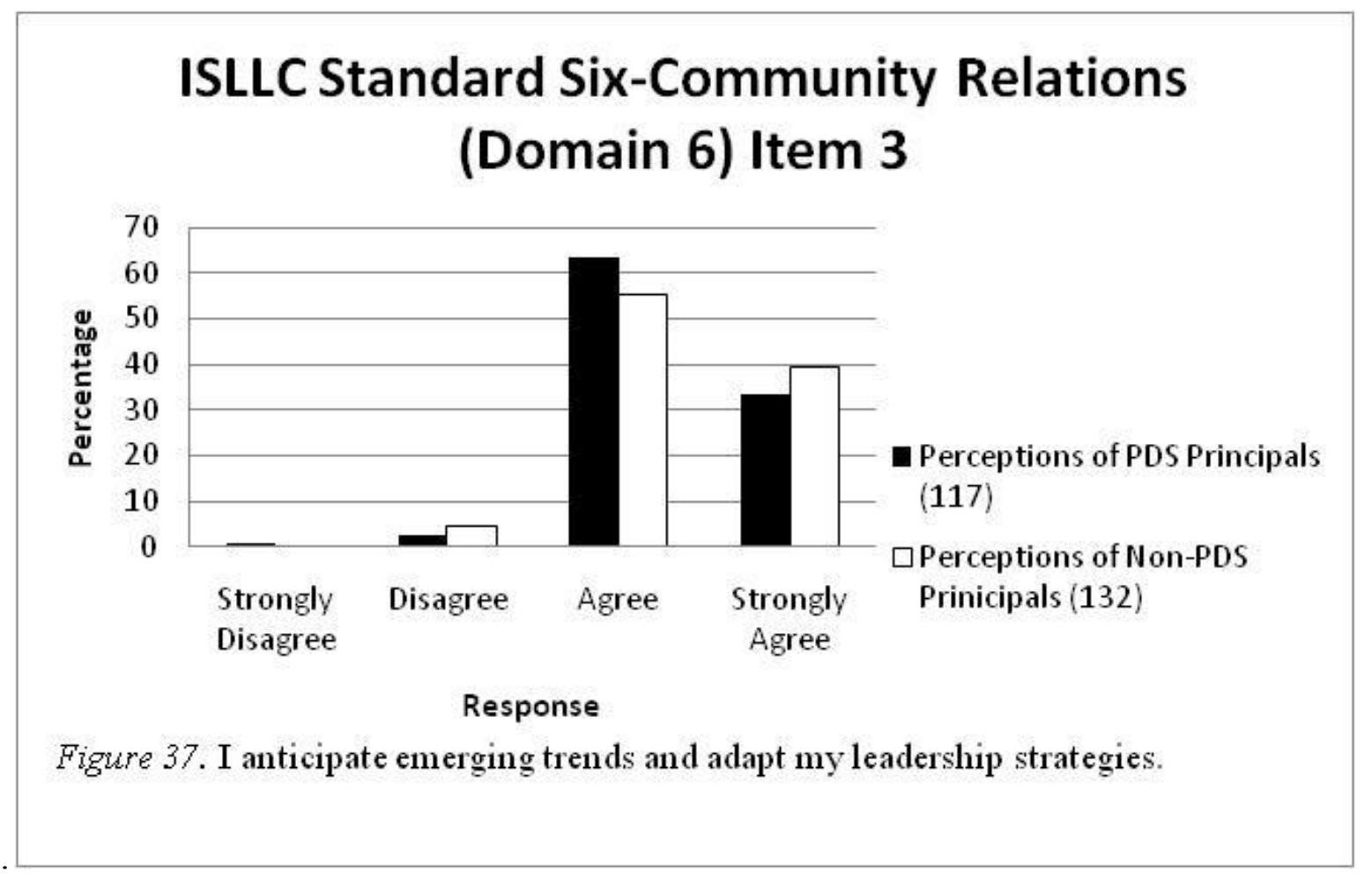

\section{Analysis of Narrative Responses}

Narrative question one. How have the Interstate School Leaders Licensure Consortium standards (ISLLC) affected your leadership? 
The responses to this question can be divided into three categories: (1) Awareness of ISLLC standards, (2) Awareness of Accountability, (3) How the ISLLC standards have affected leadership. A complete listing of all responses to Narrative Question 1 from the comparison group is available in appendix H. A complete listing of all responses from the treatment group (PDS principals) made to Narrative Question 1 is available seen in Appendix I. All comments are grouped into three related categories.

The largest number of comments to this question was in the category of how the ISLLC standards have affected leadership. In the comparison group, which consisted of principals from non-Professional Development Schools, 25 (19\%) of the respondents commented that the Interstate School Leaders Licensure Consortium standards had no influence their schoolimprovement efforts. Eleven (9\%) of the respondents in the treatment group responded that the standards had not affected their leadership.

Another disparity between the two groups was in the Awareness of ISLLC standards category. There were 19 responses from the Comparison group (non-PDS) not aware of the ISLLC standard (14\%). There were thirteen responses in the Awareness of ISLLC standards category from the treatment group (11\%).

Narrative Question Two. What do you believe are the greatest challenges for providing a culture of professional renewal inside a Professional Development School system?

The responses to this question can be divided into 5 categories:

(1) Funding,

(2) Time,

(3) Training, 
(4) Apathy and Commitment by teachers,

(5) Direction.

A complete listing of all responses from the comparison group (non-PDS) made to Question 2 divided into the five categories may be seen in Appendix J. A complete listing of all responses from the treatment group (PDS) made to Question 2 divided into the five categories may be seen in Appendix K.

The largest number of comments to this question by respondents in both groups was in the category of Time, with 25 comments from the comparison group (19\%) and 25 from the treatment group (21\%). Other categories with large numbers of responses from both groups were Training, Direction, and Apathy and Commitment by teachers.

\section{Chapter Summary}

This chapter presents the analysis of the data collected from 249 schools principals in the state of West Virginia. The purpose of this collection of data was an attempt to answer the question: Is there a statistically significant difference in the perceptions of two groups of principals regarding how leaders perceive their work as measured by an instrument aligned with the ISLLC standards?

The first section is a statistical analysis of survey responses by domain in correspondence with the ISLLC standards. The null hypothesis for each of the six domains is: There is no statistically significant difference in Domain X scores when comparing individuals who are principals of Professional Development Schools with those who are principals of nonProfessional Development Schools. The alternative hypothesis for each of the six domains is: There is a statistically significant difference in Domain X scores when comparing individuals 
who are principals of Professional Development Schools with those who are principals of nonProfessional Development Schools.

The second section of this chapter is a statistical analysis of survey responses by individual question. This is an attempt to investigate the specifics of the responses within each domain.

The third section of this chapter provides a description of the narrative responses of both the comparison and treatment groups to the two open-ended questions:

(1) How have the Interstate School Leaders Licensure Consortium standards (ISLLC) affected your leadership?

(2) What do you believe are the greatest challenges for providing a culture of professional renewal inside a Professional Development School system?

The Principal Perception Survey, designed for this study, yielded a response rate of 43.3 percent or 249 respondents. The highest number of respondents came from the comparison group (non-PDS) with 132 participants for a 53\% of the survey group. The treatment group with 117 participants (PDS) comprised 47\% of the survey group.

The results represent the responses of West Virginia Principals with active e-mail addresses who responded to the Principal Perception Survey. The numbers of male and female respondents in both groups were similar, thereby dismissing gender as a primary variable. The comparison group (non-Professional Development School Principals) was comprised of 66 males (50 percent of the comparison group) and 66 females (50 percent). The treatment group (Professional Development School Principals) was comprised of 69 males (59 percent) and 48 females (41 percent). The $t$-test for the two sample groups determined whether the observed 
differences in the scores of the two groups was larger than the difference that would result solely by chance.

At first glance, it would appear that the perceptions of the 249 West Virginia principals who responded to the survey were similar in all six domains of the survey, which was aligned with the ISLLC standards.

In all cases, no statistically significant differences were found in the six domains. In 30

of the 31 items on the survey, no statistically significant difference was found. Domain 2, Item 2 was the only item to have a statistically significant difference. The following statement is item 2: A rigorous curricular program has been collaboratively developed and is reviewed annually. The analysis of the respondents' scores for survey Item 2 in Domain 2 showed there was a statistical difference between the responses of the principals who were involved as leaders in non-Professional Development Schools compared to principals as leaders in Professional Development Schools. The scores of the principals in the treatment group (PDS principals) perceived their schools to be higher performing than the non-PDS principals. The explanation of this analysis follows.

The comparison group $\mathrm{N}$ was 132 with a mean score of 3.30 and a standard deviation of 0.589. The treatment group $\mathrm{N}$ was 117 with a mean score of 3.46 and a standard deviation of 0.550. The $t$-Test for Equality of Means showed a two-tailed significance level of 2.29, which falls outside $t$-critical; therefore, the null hypothesis is rejected. There is a significant difference in the scores of the comparison and treatment groups in Item 2. The effect size, $d$ calculation, is -0.28 , indicating a small effect of the difference made by the treatment. The negative $d$ indicates that the treatment group scores were larger than the comparison group. 
Chapter Five presents the major conclusions drawn from the analysis of the data generated by the Principal Perception Survey by domains, and by individual items. Comments from the two narrative responses are included. 


\section{Chapter Five: Discussion}

\section{Synopsis of the Study}

The problems that school leaders continue to face are the conditions connected with the leadership position that drive current principals away and thwart the ambitions of emerging school leaders. The (Professional Development School) PDS culture, by its nature, is supposed to foster professional growth for all of the professionals involved in the teaching and learning community. The professionals in PDS schools are expected to engage in the process of personal and professional reflection to ensure professional growth that is referred to as simultaneous renewal. The PDS culture evolves from all those working in the process of personal and professional reflection. These personal and professional reflections enhance the ability of the organization to achieve its goals and maintain an ongoing state of organizational renewal.

The model PDS provides a culture of collaborative support. By providing professional development opportunities and encouraging ongoing reflection in a collaborative culture of renewal, the structure of the Professional Development School could under the right circumstances support principals.

\section{Instrument Development Phase}

The Principal Perception Survey was developed to validate the research hypothesis: Professional Development Schools have an influence on how principals perceive their work in accordance with the ISLLC standards. The survey had 31 statements or questions in direct alignment with the ISLLC standards. The 575 principals were asked to indicate their level of agreement with the 31 items on a Likert-type scale, relative to their own schools. In addition, the principals were asked to respond to two opened-ended questions: 
The Principal Perception Survey was delivered electronically, and sent to the 575 principals in West Virginia with active e-mail accounts. A return rate of $43.3 \%$ was obtained with 249 responses. Of the 249 responses, 132 responses were received from non-Professional Development School principals, and 117 responses were received from Professional Development School principals (PDS). To be considered a PDS, a formal partnership agreement between a K-12 school and an institution of higher learning was required to exist. In this study, the PDS principals served as the treatment group. The split in the response rate was 53\% nonPDS principals compared to $47 \%$ PDS principals.

During the development phase, the survey instrument was analyzed for validity by a group of educators with expertise in educational leadership and related research. The reviewers confirmed the content validity during the development of the Principal Perception Survey. Content validity is the degree to which a test measures an intended content area. Content validity is determined by expert judgment (Gay, Mills, \& Airasian, 2006). These reviews constitute evidence of content validity of the Principal Perception Survey instrument. "A measure has content validity when its items accurately represent the thing (the 'universe') being measured. Content validity is not a statistical property; it is a matter of expert judgment" (Vogt, 2005, p. 59). In order to ensure construct validity, five out of thirty-one items of the survey were constructed reversely. The reverse items were brought to the principals' attention in the survey by using negative words and bold print (Susie, 1996).

The survey was designed to measure perception not practice. The survey focused on the principal's perceptions of various tasks as delineated in the ISLLC Standards. The survey did not measure the principals' depth of knowledge of the Interstate School Leaders Licensure Consortium (ISLLC) standards. 


\section{Instrument Domains Correlated to ISLLC standards}

The perceptions of principals gleaned from their responses to the 31-item survey questionnaire focused on the following six domains in direct alignment with the ISLLC standards.

- Domain (1) Visioning (ISLLC Standard 1)

An education leader promotes the success of every student by facilitating the development, articulation, implementation, and stewardship of a vision of learning that is shared and supported by all stakeholders.

- Domain (2) Advocating (ISLLC Standard 2)

An education leader promotes the success of every student by advocating, nurturing, and sustaining a school culture and instructional program conducive to student learning and staff professional growth.

- Domain (3) Management (ISLLC Standard 3)

An education leader promotes the success of every student by ensuring management of the organization, operation, and resources for a safe, efficient, and effective learning environment.

- Domain (4) Collaboration (ISLLC Standard 4)

An education leader promotes the success of every student by collaborating with faculty and community members, responding to diverse community interests and needs, and mobilizing community resources.

- Domain (5) Ethics (ISLLC Standard 5)

An education leader promotes the success of every student by acting with integrity, fairness, and in an ethical manner.

- Domain (6) Community Relations (ISLLC Standard 6) 
An education leader promotes the success of every student by understanding, responding to, and influencing the political, social, economic, legal, and cultural context.

\section{Results}

The Principal Perception Survey has painted a picture drawn from 249 principals in West Virginia. The picture shows no significant statistical relationship between perceptions of PDS principals and the perceptions of non-PDS principals when measured against the ISLLC standards when the data is compared by domain. The Principal Perception Survey found no statistically significant difference in perceptions of the treatment group (PDS principals) and the comparison group (non-PDS principals) in 30 of the 31 items.

The exception occurred in Item Two (collaboration) of Domain Two (advocating).In fact, this was the only statistically significant difference found among 31 perceptions measured. This item contained the following statement.

A rigorous curricular program has been collaboratively developed and is reviewed annually.

The respondents' scores for survey Item Two (collaboration) in Domain Two (advocating) showed there was a statistical difference between the responses of the principals who were involved as leaders in non-Professional Development Schools compared to principals as leaders in Professional Development Schools.

NCATE created a set of standards outlining a series of norms for work associated with Professional Development Schools (NCATE, 2001). Collaboration, which is standard three indicated by the National Council for Accreditation of Teacher Education (NCATE) as one part of a successful structure to maintain a PDS culture. The culture of the PDS initiative must marry the various members of the educational community allowing them to work as a cohesive unit. 
Teitel believed significant structural and ideological change must become an integral part of the institutional culture for the PDS initiative to be successful. For the PDS culture to be complete and to be an effective practice, teacher education preparation programs must be tied closely with school partners in defining parameters of best practices in teaching, administration, and research (1992). The scores of the principals in the treatment group (PDS principals) perceived their schools to be higher performing than the non-PDS principals when working collaboratively to develop a curricular program.

In addition to the 31 survey items, The Principal Perception Survey contained two questions requiring a narrative response.

Narrative question one. How have the Interstate School Leaders Licensure Consortium Standards (ISLLC) affected your leadership?

The principals' responses to this question were divided into three categories for review.

- Awareness of ISLLC standards

- Awareness of Accountability

- How the ISLLC standards have affected their leadership

A complete listing of all responses from the experimental group for question 1 is provided in Appendix I.

Awareness of ISLLC standards. Nineteen responses from the comparison group (nonPDS) indicated that those principals were unaware of the ISLLC standards (14\%). Thirteen responses in the Awareness of ISLLC standards category from the treatment group (11\%) were unaware of the ISLLC standards. The percentages indicate a difference in awareness between the two groups. In this sample, the treatment group (PDS) indicated a higher awareness of the ISLLC standards. 
Awareness of accountability. With the exception of one response, all comments from the PDS and non-PDS principals in the area of awareness of Accountability were positive. Both groups alluded to ISLLC standards as providing a basis to reflect in their practice as a leader.

ISLLC standards and leadership. The largest number of comments to Narrative Question One concerned ISLLC standards and their affect on leadership. The comparison group (non-PDS) principals' strongly felt that the ISLLC standards have not affected their leadership. Almost all of the comments stated that the ISLLC standards had no effect on their leadership practices. When the responses were analyzed, 26 of the 31 non-PDS principals stated that the ISLLC standards had either no effect or minimal effect on their leadership. In the comparison group, which consisted of principals from non-Professional Development Schools, 25 (19\%) of the respondents commented that the Interstate School Leaders Licensure Consortium standards had no influence on their school-improvement efforts. In comparison, eleven (9\%) of the respondents in the treatment group stated that the standards had not affected their leadership. The percentages indicate a difference in the narrative response of how the ISLLC standards affected leadership practices between the two groups. In this sample, the treatment group (PDS) indicated a higher usage of the ISLLC standards in leadership practices.

Narrative Question Two.

What do you believe are the greatest challenges for providing a culture of professional renewal inside a Professional Development School system?

The responses to this question were divided into 5 categories.

- Funding

- Time

- Training 
- Apathy and Commitment by teachers

- Direction

A complete listing of all responses from the experimental group (PDS) to Narrative Question 2 divided into the five categories may be seen in Appendix K. A complete listing of all responses from the treatment group (non-PDS) made to Question 2 divided into the five categories may be viewed in Appendix J.

Category: Time. The largest number of comments to this question by respondents in both groups was in the category of Time, with 25 comments from the comparison group (19\%) and 25 from the treatment group (21\%). All comments from the PDS and non-PDS principals in this category indicated that the greatest issue facing professional renewal is time. Principals from both groups described in depth how time affects and limits their leadership in the area of professional renewal.

\section{Conclusions}

The data failed to reject the null hypothesis in all six domains. The Principal Perception Survey found no significant statistical relationship between involvement with a Professional Development School and principals perceptions of their work in accordance with the ISLLC standards in all 6 domains and in 30 of the 31 items.

The exception was found in Domain Two (advocating.): Item Two (collaboration), which contained the following statement: A rigorous curricular program has been Collaboratively developed and is reviewed annually. The analysis of the respondents' scores for survey Item 2 in Domain 2 discovered a statistical difference between the responses of the principals who were involved as leaders in non-Professional Development Schools compared to principals as leaders in Professional Development Schools. The scores of the principals in the 
treatment group (PDS) indicated that they perceived their schools to be higher performing in the area of collaboration (Item 2 in Domain) than the non-PDS principals.

The PDS structure has been supportive of students and teachers. However, according to the data (principal perceptions) neither the PDS nor the non-PDS structures support principals in current leadership issues. At this time, the PDS cannot serve as a cradle for the development of principals. The conditions connected with the leadership position persist in all schools, and continue to drive principals away from the profession.

\section{Recommendations for Further Study}

There are several potential research offshoots from this work. For instance, the survey instrument used in this study could be tested for reliability and validity with other groups connected with Professional Development Schools. The survey could be used with teachers' perceptions of the PDS principal. Would the perceptions of the teacher match the perceptions of the PDS principal? The instrument could be used to measure the perception of the PDS principal through the eyes of the superintendent, student, teacher, or parent. This survey instrument could be used to formulate a 360-degree study. This type of study could lead to each survey item being reviewed for clarity and elimination of multiple points.

A 360-degree study would bring to light more information pertaining to Professional Development Schools and the leadership (principals) within those schools. The role of the school principal could be studied for potential re-structuring of responsibilities.

The Principal Perception Survey could be used in a case study of principals in PDS schools that are highly committed to the elements of the PDS culture. This study would recognize full participation in the culture of the PDS not merely a formal partnership agreement. 
A case study of principals' perceptions in schools that are involved in a PDS in their first, third and fifth years of active participation. All three schools would be identified as Professional Development Schools that are actively engaged and committed to their formal PDS partnership agreement.

In terms of instructional practices, an investigation of effective ways to use Professional Development Schools in acquiring $21^{\text {st }}$-century skills could be designed. A study of how a connection with higher education is currently being used in West Virginia schools could be investigated. A final suggestion is to investigate ways to increase community involvement in West Virginia schools.

Education has always been in a state of reformation. In the early nineteen hundreds, Dewey stated that learning was as an active process. Active knowledge leads to changes in behavior. Educational research is the exploration of knowledge that will determine the direction of educational leadership for the next generation. Quality research into as many aspects of educational leadership as possible is critically needed. The leadership of today will determine the future of education tomorrow. 


\section{References}

Abdal-Haqq, I. (1998). Professional development schools: Weighing the evidence. Thousand Oaks, CA: Corwin Press.

Anthes, K. (2002). No Child Left Behind Policy Brief: School and district leadership. Denver, CO: Education Commission of the States.

Barth, R. (1990). Improving schools from within. San Francisco, CA: Jossey-Bass.

Beck, A. (2004). The Flexner Report and the Standardization of American Medical Education. The Journal of the American Medical Association, 1-3. 291 (17).

Benedum Collaborative. (1985). Benedum Collaborative Professional Development Schools [Tree Chart]. from the Benedum Collaborative Web site:

http://www.hre.wvu.edu/benedum/pds/pds.htm

Bolman, L., \& Deal, T. (2003). Reframing organizations: Artistry, choice, and leadership. San Francisco, CA: Jossey-Bass

Book, C. (1996). Professional development schools. In J. Sikula (Ed.), Handbook of research on teacher education ( $2^{\text {nd }}$ ed., pp. 194-210). New York, NY: Macmillan Library Reference USA.

Breault, A., \& Breault, R. (2005).Experiencing Dewey: Insights for Today’s Classroom. In D. T. Hansen (Ed.). Foreword (pp. xi). Indianapolis, IN: Kappa Delta Pi.

Bush, G. (2002, January 8). President Signs Landmark No Child Left Behind Education Bill. Retrieved from The White House Web site: http://www.whitehouse.gov/news/releases/2002/01/20020108-1.html

Callahan, R., \& Button, H. (1964). Behavioral science and educational administration. Chicago: IL: University of Chicago Press. 
Campbell, R., Fleming, T., Newell, L., \& Bennion, J. (1987). A history of thought and practice in educational administration. New York, NY: Teachers College Press.

Carnegie Forum on Education and the Economy. (1987). A Nation Prepared. Washington, DC: Carnegie Forum on Education and the Economy.

Clark, D. (1998). Searching for Authentic Leadership in University Graduate Programs and with Public School Colleagues. Journal for a Just and Caring Education, 365-373.

Clark, S., \& Lewis, D. (1988). Faculty vitality: context, concerns, and prospects. In J. Smart (Ed.)., Higher education: Handbook of theory and research (Vol. IV, pp. 282-318). Bronx, New York: Agathon Press

Cotton, K. (2003). Principals and student achievement: What the research says. Alexandria, VA: Association for Supervision and Curriculum Development.

Cramer, D., \& Johnston, M. (2000). The missing voice of the principal in the school/university collaboration. In M. Johnston, P. Brosnan, D. Cramer, \& T. Dove (Eds.). Collaborative Reform and Other Improbable Dreams: The Challenges of Professional Development Schools. Albany, NY: State University of New York Press.

Creighton, T., \& Jones, G. (2001). Selection or self-selection? How Rigorous Are Our Selection Criteria for Education Administration Preparation Programs? Proceedings of the National Council of Professors of Educational Administration. University of Houston, Houston, TX.

Crowson, R., \& McPherson, R. (1987). Approaches to administrative training in education. Albany, NY: State University of New York Press.

Culbertson, J. (1965). Perspectives on educational administration and the behavioral sciences. Eugene: University of Oregon. 
Darling-Hammond, L. (Ed.). (1994). Professional development schools: Schools for developing a profession. New York, NY: Teachers College Press.

Dewey, J. (1902). The child and the curriculum. Chicago, IL: The University of Chicago Press.

Dillman, D. (2007). Mail and internet surveys: The tailored design method. 2007 update with new internet, visual, and mixed mode guide. Hoboken, NJ: John Wiley \& Sons.

Elmore, R. (2000). Building a new structure for school leadership. Washington, DC: The Albert Shanker Institute.

Field, S. (2008). How Highly Effective Professional Development School Principals Utilize Research-Based Leadership Practices To Lead The School-University Partnership (Doctoral dissertation, University of Colorado Denver, 2008). Retrieved from Dissertation Abstracts On-Line.

Fink, A. (2005). Conducting research literature reviews $\left(2^{\text {nd }}\right.$ ed.). Thousand Oaks, CA: Sage Publications.

Foster, E. S., Loving, C. C., \& Shumate, A. (2000). Effective Principals, Effective Professional Development Schools. Teaching and Change, 8(1)., 76-97.

Frasier, C. M. (1994). Two education reform trains: Standards/assessment and simultaneous renewal. Record in Educational leadership, 14(2)., 15-17.

Friedman, T. L. (2007). The world is flat, 3.0 A brief history of the twenty-first century. New York, NY: Picador.

Friedman, T. L. (2005). The world is flat. A brief history of the twenty-first century New York, NY: Farrar, Straus and Giroux.

Fullan, M. (1991). The new meaning of educational change. ( $2^{\text {nd }}$ ed.). New York, NY: Teachers College Press. 
Fullan, M., \& Hargreaves, A. (1996). What's worth fighting for in your school? New York, NY: Teachers College Press.

Fullan, M. (2004). The moral imperative of school leadership. Thousand Oaks, CA: Corwin Press.

Fullan, M., Bertani, A., \& Quinn, J. (2004, April). New Lessons for District wide Reform. Educational Leadership, 6 (1). ( pp 42-46).

Gabbard, G. (2003). Teacher Education Programs engaged in Professional Development Schools: A study of Organizational Change (Doctoral dissertation, University of Massachusetts, 2003). Retrieved from Dissertation Abstracts On-Line.

Gay, L. R., Mills, G. E., \& Airasian, P. W. (2006). Educational research: Competencies for analysis and applications 8th ed. Upper Saddle River, NJ: Pearson Education, Inc.

Goodlad, J. I. (2000). Educational in democracy: Advancing the agenda. Phi Delta Kappan, September, p. 86-89

Goodlad, J., Soder, R., et al. (1990). Places where teachers are taught. San Francisco, CA: Jossey-Bass.

Greenfield. D. (1995). Toward a theory of school administration: The centrality of leadership Educational Administration Quarterly, 31(1), 61-85

Gregg, R. T. (1960). Administration, In C.W. Harris (Ed.). Encyclopedia of educational research ( $3^{\text {rd }}$ ed., pp. 19-24). New York, NY: MacMillan

Gregg R. T. (1969). Preparation of administrators In Ebel RL (ed.). Encyclopedia of educational research, ( $4^{\text {th }}$ ed., p. 993-1004)., London, England: MacMillan.

Gullatt, D., \& Lofton, B. (1996). The principal's role in promoting academic gain. 
Natchitoches: Northwestern State University of Louisiana, Alexandria, LA: Rapides Parish Board.

Hallinger, P. (2003). Leading educational change: Reflections on the practice of instructional and transformational leadership: Cambridge Journal of Education, 33(3)., 329-351

Hallinger, P., \& Murphy, J. (1991). Developing leaders for tomorrow's schools. Phi Delta Kappan, p. 514-520.

Hallinger, P., \& Heck, R. (2002). What do you call people with visions? The role of vision, mission, and goals in school leadership and improvement. In K. Leithwood \& P. Hallinger (Eds.)., second international handbook of educational leadership and administration (p. 9-40). Alphen aan den Rijn, The Netherlands: Kluwer.

Hammonds, B. (2002, January 3). Leading and Learning for the 21stC. Quality Learning, pp. $1-9$.

Hawthorne, R. (1997). Impact on colleges of education. In N. Hoffman, W. M. Reed \& G. S. Rosenbluth (Eds.)., Lesson from restructuring experiences: stories of change in professional development schools. (p. 295-322). Albany, New York: State University of New York Press.

Hess, F. (2003). A License to Lead? A New Leadership Agenda for America's Schools. (Prepared as part of the $21^{\text {st }}$ century Schools Project). Washington, DC: Progressive Policy Institute.

Hunkins, F., Wiseman, D., \& Williams, R. (1995). Supporting collaborative inquiry. In R.T Osguthorpe, M.F Harris, R, C. Harris, \& S. Black (Eds.)., Partner schools: Centers for educational renewal (p. 99-123). San Francisco, CA: Jossey-Bass. 
Interstate School Leaders Licensure Consortium. (2006). Interstate School Leaders Licensure Consortium: Standards for School Leaders. Washington, DC: Council of Chief State School Officers.

Keller, B. (2000, May 3). Building On Experiences. Journal of Education Week. 19(34)., 36-40 Retrieved January 22, 2009, from http://www.bigpicture.org/2000/05/building-onexperience/

Kersh, P., \& Mastal, J. E. (1998). An analysis of studies of collaboration between universities and K-12 schools. Educational Forum, 62, 218-225.

Kess, D. (2002). The role of school-based site coordinator in Professional Development Schools: The Impact on Teacher Professionalism (Doctoral dissertation, University of Connecticut, 2002). Retrieved from Dissertation Abstracts On-Line.

Klaumeier, R. L. (1990). Four decades of calls for reform of teacher education: The 1950s through the 1980s. Teacher Education Quarterly. 17(4). 23-64

Lashway, L. (2002, November). Trends in School Leadership. Retrieved from Eric Digest Web site: http://eric.uoregon.edu/publications/digests/digest162.html

Leithwood, K. (2004). Educational leadership: A review of the research. The Laboratory for Student Success. Philadelphia, PA: The Mid-Atlantic Regional Educational Laboratory at Temple University Center for Research in Human Development and Education

Leithwood, K., Louis Seashore, K., Anderson, S., \& Wahlstrom, K. (2004). How leadership influences student learning: The Wallace Foundation http://www.wallacefoundation.org/KnowledgeCenter/KnowledgeTopics/CurrentAreasofF ocus/EducationLeadership/Pages/HowLeadershipInfluencesStudentLearning.aspx 
Levine, A. (2005a, March). Educating School Leaders. Washington, DC: Education Schools Project.

Levine, A. (2005b, April 15). Point of View: Change in the Principal's Office: the role of Universities. Retrieved from The Chronicle of Higher Education Web site: http://www.ucop.edu/acadinit/mastplan/edd/che_review04152005.htm

Levine, M. (1992). Professional practice schools: Linking teacher education and school reform. New York, NY: Teachers College Press.

Likert, R. (1967). The human organization: Its management and value. New York, NY: McGraw Hill Book Company.

Lyons, N., Stroble, B., et al. (1997). The idea of the university in an age of school reform: the shaping force of professional development schools. In R. Trachtman (Ed.)., Making professional development schools work (p. 88-111). New York, NY \& and London, England: Teachers College Press

Marzano, R., McNulty, B., \& Waters, T. (2005). School leadership that works: From research to results. Aurora, CO.

Matthews, L. J., \& Crow, G. M. (2003). Being and becoming a principal: Role conceptions for contemporary principals and assistant principals. Boston, MA: Pearson Education.

McCarthy, M. M., \& Kuh, G. D. (1997). Continuity and change: The educational leadership professoriate. Columbia, MO: The University Council for Educational Administration.

McCarthy, M.M., Kuh G.D., Newell L.J., \& Iacona C.M., (1988). Under Scrutiny: The Educational Administration Professorate, Tempe, AZ: University Council for Educational Administration.

Metcalf-Turner, P. (1999). Variable Definitions of Professional Development Schools: A 
Desire or a Dilemma? Peabody Journal of Education, 74(3\&4)., 33-41.

Miklos, E. (1983, 3 19). Evolution in administrator preparation programs. Educational Administration Quarterly, pp. 153-177.

Milstein, M., \& Associates. (1993). Changing the way we prepare educational leaders: The danforth experience. Newbury Park: CA Corwin Press.

Mitchell, L. (1950). Our children and our schools. New York: Simon \& Schuster

Murnane, R., \& Levy, F. (1996). Teaching the new basic skills: Principles for educating children to thrive in a changing economy. New York: Free Press.

Murphy, J. (1990). The educational reform movement of the 1980s: A comprehensive analysis. In J. Murphy (Ed.)., The reform of america public education in the 1980s: Perspectives and cases (p. 3-55). Berkeley, CA: McCutchan.

Murphy J. (1992). The landscape of leadership preparation: Reframing the education of school administrators. Newbury Park: Corwin Press.

Murphy, J. (1998). Preparation for the school principalship: The United States' story. School Leadership \& Management, 359-372.

Murphy, J. (2005a). Connecting teacher leadership and school improvement. Thousand Oaks: Corwin Press.

Murphy, J (2005b). Unpacking the Foundations of ISLLC Standards and Addressing Concerns in the Academic Community. Educational Administration Quarterly, 41(1)., 154-191

Murphy, J. (2006, fall). PERSPECTIVE: Of Dubious Efficacy-Questioning the Core of University-based Preparation Programs for School Leaders. School Leadership News. 17(6).

Murphy, J., \& Hallinger, P. (1987). Approaches to administrative training in education. 
Albany: State University of New York Press.

Murphy, J., \& Shipman, N. (1996, November 2). Interstate School Leaders Licensure Consortium standards for School Leaders. Retrieved from The Wallace Foundation website: http://www.wallacefoundation.org/NR/rdonlyres/A68DC3DE-D3DD-46BBBCC713DA0948A1B6/0/isllcstd.pdf

Murphy, J. \&. Shipman, N. (1996). Interstate School Leaders Licensure Consortium. Council of Chief State School Officers (pp. 6-12). Washington: Council of Chief State School Officers.

Murphy, J., \& Shipman, N. (1999). The Interstate School Leaders Licensure Consortium: A Standards - Based Approach t Strengthening Educational Leadership. Personnel Evaluation in Education, 205-224.

National Commission on Excellence in Educational Administration (1987). Leaders for America's Schools, Tempe, AZ: University Council for Educational Administration.

National Commission on Teaching and America's Future. (1996). What matters most: teaching for America's future. New York: National Commission on Teaching and America's Future.

National Council for Accreditation of Teacher Education. (2001). Draft standards for Identifying and supporting quality professional development schools. Washington DC: National council for the accreditation of teacher education.

National Society for the Study of Education. (1964). Behavioral science and educational administration. Chicago: University of Chicago Press. 
New York Times. (1917, February 27). Keep Schools Free From Money Taint. New York Times. Retrieved from http://query.nytimes.com/gst/abstract.html?res=950CE1DF 123BEE3ABC4951DFB466838

Norlander-Case, K. A., Reagan, T. G., \& Case, C. W. (1999). The professional teacher: The preparation and nurturance of the reflective practitioner (Vol. 4). San Francisco, CA: Jossey-Bass Publishers.

Pearlman, B. (2006). 21st Century Learning in Schools-A case Study of New Technology High School in Napa, CA. New Direction for Youth Development .

Pellicer, L. O., \& Anderson, L. W. (1995). A handbook for teacher leaders. Thousand Oaks: Corwin Press.

Perrone, V. (1989). Working papers reflections on teachers, schools, and communities. New York: Teachers College Press.

Portney, L., \& Watkins, M. (2000). Foundations of clinical research applications to practice. Upper Saddle River: Prentice-Hall, Inc.

Richardson, S. W. (1990). The professional development school: A common sense approach to improving education. Fort Worth: Sid W. Richardson Foundation.

Richardson, S. W. (1993). The professional development school: A common sense approach to improving education. Fort Worth: Sid W. Richardson Foundation.

Robinson, S., \& Darling-Hammond, L. (1994). Change for collaboration and collaboration for change: transforming teaching through school-university partnerships. In L. DarlingHammond (Ed.)., Professional development schools: schools for developing a profession. New York: Teachers College Press. 
Ryan, A. (1995). John Dewey and the high tide of american liberalism. New York: W. W. Norton \& Company.

Salaiz, N. (2004). A Multiple Case Study of a Select Group of Texas School Administrators' Perceptions of a Change Initiative; Tech Prep Integration into Career and Technology Education (CATE). Programs (Doctoral dissertation, University of Texas at Austin, 2004). Retrieved from Dissertation Abstracts On-Line.

Schon, D. A. (1983). The reflective practitioner. How professionals think in action. New York: Basic Books.

Schugurensky, D. (2002, September 15). Selected Moments of the $20^{\text {th }}$ Century: Retrieved from Department of Adult Education, Community Development and Counseling Psychology Web site: http://www.wier.ca/ daniel_schugurens/assignment1/1931addams.html Schaefer, R. (1967). The school as a center of inquiry. New York: Harper \& Row Publishers. Sewell, T. E., Shapiro, J. P., \& Sanford, J. S., (1995). Professional development schools in the inner city: Policy implications for school-university collaboration. In H. G. Petrie (Ed.)., Professionalization, partnership, and power: Building professional development schools (pp. 179-198). Albany: State University of New York Press.

Silins, H. C., Mulford, W. R., \& Zarins, S. (2002). Organizational learning and school change. Educational Administration Quarterly, 38(5)., 613-642

Silver, P.F. (1982). Administrator preparation in Mitzel Hem (ed.). Encyclopedia of Educational Research, $5^{\text {th }}$ ed., Vol. 1, p. 49-59, New York: Free Press.

Smith, Mary. (2000). Who was Lucy Sprague... and why should you know? In ProQuest Information and learning Company online. Retrieved July 31, 2008, from http://findarticles.com/p/articles/mi_qa3614/is_200020/ai_n8907594/print?tag=artBody;coll 
Stallings, J., \& Kowalski, T. (1990). Research on professional development schools. In R. Houston (Ed.)., Handbook of research on teacher education. New York, New York Macmillan.

Suskie, L. (1996). Questionnaire survey research: What works ( ${ }^{\text {nd }}$ Ed.). Tallahassee, FL: Association for Institutional Research.

Teitel, L. (1992). The Impact of Professional Development School Partnerships on the Preparation of Teachers. Teaching Education, 4 (2)., pp. 77-85.

Teitel, L. (1996). Professional development schools: a literature review. Boston: Professional Development School Standards Project, National Council for Accreditation of Teacher Education.

Teitel, L. (1999). Looking toward the future by understanding the past: The historical context of professional development schools. Peabody Journal of Education, 74, 6-20.

Teitel, L. (2003). The professional development schools handbook. Thousand Oaks, CA: Sage Publications Company.

Tilford, K. (2007). The essence of the principal's role in a professional development school (Doctoral dissertation, University of Florida, 2007).

Trachtman, R., \& Levine, M. (1997). Reinventing Leadership in Professional Development Schools. In R. Trachtman and M. Levine (Eds.)., Making Professional Development Schools Work: Politics, Practice, and Policy. New York, New York: Teacher's College Press.

The Holmes Group. (1986). Tomorrow's teachers: A report of the Holmes Group. East Lansing, MI: Holmes Group 
The Holmes Group. (1990). Tomorrow's schools: principles for the design of professional development schools: a report of the Holmes Group. East Lansing, MI (501 Erickson Hall, East Lansing 48824-10349).: Holmes Group.

The Holmes Group. (1995). Tomorrow's schools of education: a report of the Holmes Group. East Lansing Mich.: Holmes Group

The National Commission on Excellence in Education (1983, April). A Nation at Risk: The Imperative for Educational Reform. Retrieved March 13, 2008 from http://www.ed.gov/pubs/NatAtRisk/title.html

Thomson, S. (1998). Causing change: the national policy board for educational administration. Newbury Park: Corwin Press.

Vogt, P. W. (2005). Dictionary of statistics \& methodology; A nontechnical guide for the social sciences. Thousand Oaks: Sage Publications, Inc.

Woolfolk, A (2007). Educational psychology. Boston, MA: Pearson.

Yuk1, G. (1994). Leadership in organizations ( $3^{\text {rd }}$ ed.). Englewood Cliffs, NJ: Prentice Hall 
Appendix A

Principal Perception Survey (p. 1)

\begin{tabular}{|c|c|c|c|c|}
\hline \multicolumn{5}{|c|}{$\begin{array}{l}\text { Leaders of West Virginia Professional Development Schools: } \\
\text { Principal Perception Survey }\end{array}$} \\
\hline \multicolumn{5}{|c|}{ NOTE: All respondents will remain anonvmous. } \\
\hline $\begin{array}{l}\text { Principal of a: (select on } \\
\text { (PDS) Professiona } \\
\text { Development School (De } \\
\text { by having a Formal Par } \\
\text { agreement) } \\
\text { non-Professional } \\
\text { Development School }\end{array}$ & $\begin{array}{l}\text { Principal of a: (select one) } \\
\text { (PDS) Professional } \\
\text { Development School (Defined } \\
\text { by having a Formal Partnership } \\
\text { agreement) }\end{array}$ & \# Years as a & Principa & \\
\hline \multicolumn{5}{|c|}{$\begin{array}{l}\text { Directions: Listed below are statements about the six areas related to the Educational Leadership Policy } \\
\text { Standards (ISLLC) } \\
\quad \text { Please select the number at the right of each statement that best describes your agreement with } \\
\text { the idea in the statement. }\end{array}$} \\
\hline \multicolumn{5}{|l|}{ Domain 1: Visioning } \\
\hline & $\begin{array}{c}1 \\
\text { Strongly } \\
\text { Disagree }\end{array}$ & $\begin{array}{c}2 \\
\text { Disagree }\end{array}$ & $\begin{array}{c}3 \\
\text { Agree }\end{array}$ & $\begin{array}{l}4 \\
\text { Strongly } \\
\text { Agree }\end{array}$ \\
\hline $\begin{array}{l}\text { 1. I consider myself a good collaborator when it comes to } \\
\text { developing a shared vision for this school. }\end{array}$ & 1 & 2 & 3 & 4 \\
\hline $\begin{array}{l}\text { 2. I do not collect and use data to identify goals or assess } \\
\text { organizational effectiveness. }\end{array}$ & 1 & 2 & 3 & 4 \\
\hline $\begin{array}{l}\text { 3. I consider myself a methodical planner when it comes to the } \\
\text { development of school goals. }\end{array}$ & 1 & 2 & 3 & 4 \\
\hline $\begin{array}{l}\text { 4. I often deliver the message that continuous improvement is } \\
\text { necessary to achieve goals. }\end{array}$ & 1 & 2 & 3 & 4 \\
\hline $\begin{array}{l}5 . \text { I often include stakeholder groups when assessing whether or } \\
\text { not we have achieved goals that move us toward the shared } \\
\text { vision. }\end{array}$ & 1 & 2 & 3 & 4 \\
\hline \multicolumn{5}{|l|}{ Domain 2: Advocating } \\
\hline & $\begin{array}{c}1 \\
\text { Strongly } \\
\text { Disagree }\end{array}$ & \begin{tabular}{c|c|}
2 \\
Disagree
\end{tabular} & $\begin{array}{c}3 \\
\text { Agree }\end{array}$ & $\begin{array}{c}4 \\
\text { Strongly } \\
\text { Agree }\end{array}$ \\
\hline $\begin{array}{l}\text { 1. My faculty and I have developed and maintained high } \\
\text { expectations for all students. }\end{array}$ & 1 & 2 & 3 & 4 \\
\hline
\end{tabular}




\section{Appendix A}

\section{Principal Perception Survey (p. 2)}

\begin{tabular}{|c|c|c|c|c|}
\hline $\begin{array}{l}\text { 2. A rigorous curricular program has been collaboratively } \\
\text { developed and is reviewed annually. }\end{array}$ & 1 & 2 & 3 & 4 \\
\hline $\begin{array}{l}\text { 3. I believe we have a structured motivational learning } \\
\text { environment at this school. }\end{array}$ & 1 & 2 & 3 & 3 \\
\hline 4. I have a plan for supervising instruction and I follow it. & 1 & 2 & 3 & 4 \\
\hline $\begin{array}{l}\text { 5. I do not believe our current assessment system is structured } \\
\text { for student growth. }\end{array}$ & 1 & 2 & 3 & 4 \\
\hline $\begin{array}{l}\text { 6. I believe it is important to develop the instructional capacity } \\
\text { of staff. }\end{array}$ & 1 & 2 & 3 & 4 \\
\hline 7. My faculty, staff, and I value and protect instructional time. & 1 & 2 & 3 & 4 \\
\hline $\begin{array}{l}\text { 8. We are constantly engaged in discussions about the most } \\
\text { effective instructional technologies, and we seek ways to support } \\
\text { getting and using it. }\end{array}$ & 1 & 2 & 3 & 4 \\
\hline $\begin{array}{l}\text { 9. Evaluation of instructional program impact is done } \\
\text { continuously and the program is modified daily to meet students' } \\
\text { needs. }\end{array}$ & 1 & 2 & 3 & 4 \\
\hline \multicolumn{5}{|l|}{ Domain 3: Management } \\
\hline & $\begin{array}{c}1 \\
\text { Strongly } \\
\text { Disagree }\end{array}$ & \begin{tabular}{c|}
2 \\
Disagre \\
$\mathrm{e}$
\end{tabular} & $\begin{array}{c}3 \\
\text { Agree }\end{array}$ & $\begin{array}{l}4 \\
\text { Strongly } \\
\text { Agree }\end{array}$ \\
\hline $\begin{array}{l}\text { 1. I constantly review operational systems to determine if they } \\
\text { are meeting the needs of all stakeholders. }\end{array}$ & 1 & 2 & 3 & 4 \\
\hline $\begin{array}{l}\text { 2. I challenge my faculty and staff to find ways to maximize } \\
\text { resources. }\end{array}$ & 1 & 2 & 3 & 4 \\
\hline $\begin{array}{l}\text { 3. Safety policy and procedures are at the forefront of } \\
\text { continuous review. }\end{array}$ & 1 & 2 & 3 & 4 \\
\hline $\begin{array}{l}\text { 4. I develop leadership capacity in my faculty and staff by } \\
\text { enabling them to solve organizational problems. }\end{array}$ & 1 & 2 & 3 & 4 \\
\hline 5. Focused teaching and learning time is built into logistics. & 1 & 2 & 3 & 4 \\
\hline \multicolumn{5}{|c|}{ Domain 4: Collaboration } \\
\hline & $\begin{array}{c}1 \\
\text { Strongly } \\
\text { Disagree }\end{array}$ & \begin{tabular}{c|}
2 \\
Disagree
\end{tabular} & $\begin{array}{c}3 \\
\text { Agree }\end{array}$ & $\begin{array}{l}4 \\
\text { Strongly } \\
\text { Agree }\end{array}$ \\
\hline $\begin{array}{l}\text { 1. The faculty and I analyze data pertinent to the educational } \\
\text { environment. }\end{array}$ & 1 & 2 & 3 & 4 \\
\hline $\begin{array}{l}\text { 2. I expect faculty and staff members to welcome and engage } \\
\text { the community. }\end{array}$ & 1 & 2 & 3 & 4 \\
\hline
\end{tabular}


Appendix A

Principal Perception Survey (p. 3)

\begin{tabular}{|c|c|c|c|c|}
\hline $\begin{array}{l}\text { 3. The well being of students and their families give us a } \\
\text { common purpose for building positive relationships. }\end{array}$ & 1 & 2 & 3 & 4 \\
\hline $\begin{array}{l}\text { 4. I don't waste my time reaching out to disinterested } \\
\text { community partners. }\end{array}$ & 1 & 2 & 3 & 4 \\
\hline \multicolumn{5}{|l|}{ Domain 5: Ethics } \\
\hline & $\begin{array}{c}1 \\
\text { Strongly } \\
\text { Disagree }\end{array}$ & $\begin{array}{c}2 \\
\text { Disagree }\end{array}$ & $\begin{array}{c}3 \\
\text { Agree }\end{array}$ & $\begin{array}{l}4 \\
\text { Strongly } \\
\text { Agree }\end{array}$ \\
\hline $\begin{array}{l}\text { 1. I do not think it is important that every student maximizes } \\
\text { his/her achievement or success. }\end{array}$ & 1 & 2 & 3 & 4 \\
\hline 2. I model the kind of behavior I expect from everyone else. & 1 & 2 & 3 & 4 \\
\hline $\begin{array}{l}\text { 3. My vision for building a fear free culture of teaching and } \\
\text { learning is centered by democratic principles, equality and } \\
\text { fairness, and strength in diversity. }\end{array}$ & 1 & 2 & 3 & 4 \\
\hline $\begin{array}{l}\text { 4. Legal consequences of decision making are not connected to } \\
\text { moral decision making. }\end{array}$ & 1 & 2 & 3 & 4 \\
\hline $\begin{array}{l}\text { 5. I base all my leadership work on opening the door of } \\
\text { education to all people regardless of social status, ethnicity, } \\
\text { gender, religion, and cultural background. }\end{array}$ & 1 & 2 & 3 & 4 \\
\hline \multicolumn{5}{|l|}{ Domain 6: Community Relations } \\
\hline 't & $\begin{array}{c}1 \\
\text { Strongly } \\
\text { Disagree }\end{array}$ & $\begin{array}{l}2 \\
\text { Disagre } \\
\mathrm{e}\end{array}$ & $\begin{array}{c}3 \\
\text { Not } \\
\text { Sure }\end{array}$ & $\begin{array}{l}4 \\
\text { Strongly } \\
\text { Agree }\end{array}$ \\
\hline 1. I and my faculty advocate for children and their families. & 1 & 2 & 3 & 4 \\
\hline 2. I act to influence policies affecting student learning. & 1 & 2 & 3 & 4 \\
\hline $\begin{array}{l}\text { 3. I anticipate emerging trends and adapt my leadership } \\
\text { strategies. }\end{array}$ & 1 & 2 & 3 & 4 \\
\hline
\end{tabular}


Appendix B

Letter of Support

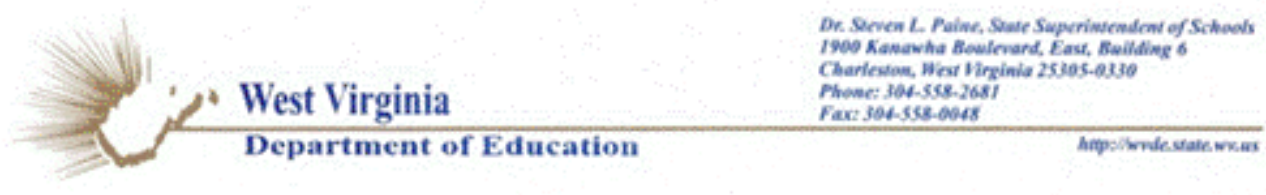

April 13, 2009

Mr. David Lancaster

Assistant Professor of Education

Student Teacher Supervisor

Education Division

West Virginia University-Parkersburg

300 Campus Drive

Parkersburg. WV 26104-8647

Dear Mr. Lancaster:

I am most happy to support your disseriation study in the areas of leadership and the connection with Professional Development Schools. The study of the relationship between involvement with Professional Development Schools and how leaders pereeive their work in alignment with the Interstate School Leaders Licensure Consortium (ISLLC) standards is an interesting topic.

As I understand your study, you will be gathering perceptual data from principals of Professional Development Schools and Non-Professional Development Schools in alignment with the ISLLC standards. I look forward to reading the results of your study which has the potential for important contributions to current knowledge on leadership in West Virginia.

You are welcome to contact any of my staff involved with this work. Dr. Karen Huffman can provide you valuable information about the leadership in public schools across the state of West Virginia. I know all of the other staff members will be pleased to discuss this work and provide you with any information you might request. Dr. Huffman or a member of her staff may be reached at 304-558-3199.

If 1 can be of further assistance, please feel free to contact me. Best wishes in your dissertation study.

Sineerely,

State Superintendent of Schools

DEST VIEGTI HOLRD OF FBICATON

20es:-2004

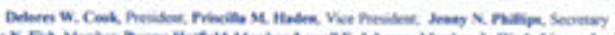

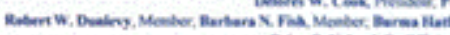

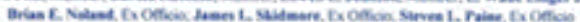




\author{
Appendix C \\ Principal Advance E-mail
}

\title{
Dear Principal,
}

I am requesting your assistance. My name is David Lancaster, and I am involved with the Leadership Doctoral Cohort at West Virginia University. The completion of this project is the final step in obtaining my Doctor in Education Leadership.

This research is focused on the problem of school leadership conditions that drive current leaders away and thwart the ambitions of emerging school leaders. The statistics for this investigation will come from the data collected from current principals in the state of West Virginia. This project has the support of Dr. Paine, State Superintendent of Schools.

You will receive a questionnaire package via e-mail, which will consist of thirty-one questions with the responses rated from 1-4. The number one response starts out with the opinion of strongly agreeing with a statement. The number two represents agreement with the statement while the number three supports disagreement with the statement. The number four represents strong disagreement with the statement.

I sincerely appreciate your time in filling out the survey. All individual responses will be kept confidential. There will be no penalty for anyone choosing not to participate. During the survey, you do not have to answer all of the questions and may quit at any time.

To ensure all procedures are followed The West Virginia University Institutional Review Board (IRB) has acknowledgment of this study on file. I will serve as the Principal Investigator under the direct supervision of Dr. Paul Chapman from WVU for this project. My affiliation with WVU is my involvement as a student in the Leadership Doctoral Cohort program. Dr. Chapman may be reached at 304-293-2174 or Paul.Chapman@mail.wvu.edu.

If you have any questions about this project, please contact me at my home phone number. (304684-3265) In addition, upon completion of this project, I will be glad to send you a copy of the survey results per your request.

Sincerely,

David L Lancaster

dave.lancaster@mail.wvu.edu

304-684-3265 
Appendix D

Participation Request

Dear Principal,

This is the research survey I discussed in a prior e-mail. Again, thank you for taking time out of your busy schedule to help me with my project. This is a voluntary research survey and there will be no penalty for anyone choosing not to participate. During the survey, you do not have to answer all of the questions and may quit at any time.

This survey contains thirty-one questions with the responses rated from 1-4. The number one response starts out with the opinion of strongly agreeing with a statement. Number two represents agreement with the statement while number three supports disagreement with the statement. Number four represents strong disagreement with the statement. All individual responses will remain anonymous.

If you have any questions about this project, please contact me at my home phone number. (304-684-3265) In addition, upon completion of this project, I would be glad to send you a copy of the survey results per your request.

Sincerely,

David L Lancaster

dave.lancaster@mail.wvu.edu

304-684-3265 
Appendix E

Follow up E-mail 1

Dear Principal,

I'm following up on a research survey request made a few days ago. Please take the time to fill out the survey on Principals' perceptions. I'm eager to finish gathering the information and complete the report. If you need any assistance, please contact me. I want to ensure you that all individual responses will be kept confidential.

Again, thank you for taking time out of your busy schedule to help me with my project. This is a voluntary research survey and there will be no penalty for anyone choosing not to participate. During the survey, you do not have to answer all of the questions and may quit at any time.

Thank you for the time and effort in this matter.

Sincerely,

David L Lancaster

dave.lancaster@mail.wvu.edu

304-684-3265 
Appendix F

Follow up E-mail 2

Dear Principal,

I'm following up on a research survey request made a few days ago. Please take the time to fill out the survey on Principals' perceptions. This project has the support of Dr. Paine, State Superintendant of Schools. If you need any assistance, please contact me. I want to ensure you all individual responses will be kept confidential.

Again, thank you for taking time out of your busy schedule to help me with my project. This is a voluntary research survey and there will be no penalty for anyone choosing not to participate. During the survey, you do not have to answer all of the questions and may quit at any time. Thank you for the time and effort you have put into this matter. I have sent a copy of the prior survey via e-mail for your convenience.

Sincerely,

David L Lancaster

dave.lancaster@mail.wvu.edu

304-684-3265 


\section{Appendix G}

Follow up E-mail 3

\section{Dear Principal,}

I'm following up on a survey request made a few days ago. Please take the time to fill out the survey on Principals' perceptions. I'm eager to finish gathering the information and complete the report. This phone message will be your last reminder. If you need any assistance, please contact me. I want to ensure you that all individual responses will be kept confidential.

Again, thank you for taking time out of your busy schedule to help me with my project. This is a voluntary research survey and there will be no penalty for anyone choosing not to participate. During the survey, you do not have to answer all of the questions and may quit at any time. Thank you for the time and effort in this matter.

Sincerely,

David L Lancaster

dave.lancaster@mail.wvu.edu

304-684-3265 
Appendix $\mathrm{H}$

Narrative Question 1: Non-PDS (p. 1)

Complete listing of all responses from the comparison group made to Question 1(non-PDS)

How have the Interstate School Leaders Licensure Consortium standards (ISLLC) affected your leadership?

\section{Awareness of ISLLC standards}

- ?

- ???

- ??????????????????

- Am not aware of this??

- Do not know of this consortium.

- I am not aware of this.

- I am not familiar with ISLLC and have no idea what it is.

- I am not familiar with ISLLC standards

- I am not sure what that is???

- I am not sure.

- I am unaware of them.

- I am unaware of these standards. No information has been shared on this topic within this specific titled program definition.

- I have no knowledge of the ISLLC

- I used the standards to organize my portfolio when I graduated from the master's program.

- It is my intent to keep it updated under those standards. I am also a National Board Certified Teacher and I am eagerly awaiting the Principal Certification. I will be interested in seeing

- How the standards combine or compare.

- Never heard of it!

- Never heard of them :(

- Not familiar with the standards

\section{Awareness of Accountability}

- I have tried to utilize the professional communities system within my building through book Studies and subject area discussions. This takes time and commitment on my part.

- ?

- Provided a basis to reflect on my practice. 
Appendix $\mathrm{H}$

Narrative Question 1: Non-PDS (p .2)

- The emphasis on success for every student and marshaling the resources and community to provide such success are integral to my practice as a school leader.

- The ISLLC have given school administrators across the nations a basic framework of what school administrators need to know in order to effectively and efficiently administer and Manage a school from Florida to Washington State. We need a national boardcertified program for administrators much like we have for our teachers.

- ???

- They are the guidepost for developing a strong and effective leadership.

- They have a caused a refocusing on what schooling is all about and and redirected many of my efforts into more productive practice

\section{How the ISLLC standards have affected leadership}

- Have not affected my leadership

- I apologize, but I have not used this resource.

- I don't know that they have affected my leadership.

- I don't know what the standards are nor am I familiar with the Consortium. Therefore, they have had no influence on my leadership.

- I have not looked at these standards.

- It has not

- It has not been a concern of mine, I am looking at retirement.

- It has not.

- It has not. I do what I know works and stick with proven leadership standards and skills.

- It hasn't affected my leadership. I have never heard of the ISLLC.

- Minimally

- My leadership is based on training and vision with lifelong learning. I am not familiar with the

- Standards except when I took the long version of the praxis years ago for PA certification.

- $\mathrm{n} / \mathrm{a}$

- $\mathrm{n} / \mathrm{a}$

- No impact! When I was in the county, I was unaware of them.

- None 


\author{
Appendix $\mathrm{H}$ \\ Narrative Question 1: Non-PDS (p .3)
}

- NONE

- None

- None

- not applicable

- Not at all.

- Not at all.

- Not to a large part at this time. May have more and more influence as time passes.

- Our county has provided us with great professional development. I can't say much about this

- I am not very up-to-date on it.

- These standards have always been in place in Kanawha County.

- They have had impact only at the time of my study in the program at WVU. Our county does not refer to them but does provide on-going professional development through Leadership

- ???

- They have not.

- Up to this point, I don't know that they have. 
Appendix I

Narrative Question 1: PDS (p 1)

Complete listing of all responses from the experimental group made to Question 1(PDS)

How have the Interstate School Leaders Licensure Consortium standards (ISLLC) affected your leadership?

\section{Awareness of ISLLC standards}

- ?

- ??

- I am not aware of this consortium.

- I am not aware of this movement.

- I am not familiar with the ISLLC.

- I am not familiar with these standards.

- I am unfamiliar with this program.

- I have no idea what this is.

- I have not seen the ISLLC standards.

- If I am aware of this, it is under a different name.

- If I knew what the ISLLC was, I would tell you.

- Not aware of this Consortium.

- Sorry, I am not familiar with these standards.

\section{Awareness of Accountability}

- Awareness of Accountability.

- I assumed that Monongahela Elementary would be classified as a Professional Development School because of our partnership with Fairmont State University.

- The ISLLC has not really affected my leadership in that they are a list of "common sense" goals

- For an educational administrator as opposed to a building administrator. Plus, I came into my position with a strong PD background and a background as a teacher leader with 28 years

- ????

- Experience. The ISLLC would be extremely helpful tool to those going into administration early in their careers.

- ????? 
Appendix I

Narrative Question 1: PDS (p 2)

- The standards are known nationally and allow WV principals to compare our work to principals nationally.

- They are the basis for all that is done at the leadership level.

\section{How the ISLLC standards affected your leadership}

- Enhanced them

- I am familiar with these and incorporate these standards into my leadership practice.

- I have not really used those standards. I have always tried to follow the requirements of our

- State for qualities and responsibilities for administrators, used the 7 correlates for effective schools, and tried to stay abreast of new developments, and constantly look at what is good for Students. Above all, we must stay student centered.

- ????????

- It has not.

- It has provides me with a framework on which to guide teachers.

- It is a good framework to use to guide your planning and reflection on your work.

- No

- Not that I am aware.

- No effect.

- No.

- None

- None, for my skills are well developed.

- Not at all, thus far.

- Somewhat

- They are a guide.

- Modules to keep us current on trends and developments that not only affect our role but the Students we serve.

- ?

- They haven't

- Very limited affect

- We have an awareness of the standards and seek to assist the school and community to reach those standards. 


\section{Appendix $\mathbf{J}$}

Narrative Question 2: Non-PDS (P. 1)

Complete listing of all responses from the comparison group made to Question 2 (non-PDS)

What do you believe are the greatest challenges for providing a culture of professional renewal inside a Professional Development School system?

\section{Funding}

- Funding and Time for teachers to practice what they have learned and discuss with collaboration.

- Limited Resources

- Money and time.

\section{Time}

- TIME!!!!!!!!!

- Time to work with staff as the staff is overburdened with new initiatives every year after we are told there will be no new initiatives.

- TIME--There is never enough time to do everything we want or need to do. 2) MORALE--Teacher and general staff morale are at their lowest right now.

- Time !! While this culture is important to developed, it often takes away time from the business of being in the school to accomplish the workload there. We are told that being an instructional leader is our main focus and I agree that it should be. In reality, it slides down the scale because of real world problems of plant and community management and engagement issues.

- Time and money

- Time

- Time, seems to be the greatest problem each of us faces. We need more time to accomplish the goals and plan.

- time allotment, continuity, monetary support, effective training, follow-up

- Time

- TIME and MONEY! To have the time to plan for staff development that will be used for student learning, thus money to pay for substitute teachers so planning can take place. 


\section{Appendix J}

Narrative Question 2: Non-PDS (P. 2)

- Finding the time and resources to provide engaging and meaningful Professional Dev. that has real world application and pulls teachers and staff along with admin. into the learning process willingly. If it is truly important let's make sure that we budget the time to do it and pay staff accordingly to do the training.

- TIME, TIME, AND TIME - I live in Virginia where the teachers get 7 full professional development days at the beginning of each year and have to gain 12 hours of technology professional development each year. my daughters Standards of Learning scores were advanced in ALL areas. Our teachers are just as good, just (if not more) dedicated to student success - they are not given enough support.

- finding time to do the PD activities

- Time!

- TIME! TIME! TIME! and by the way did I mention TIME! At the highest levels, we must address the all too common idea that each discipline or current stakeholder is the most important and can demand first place. Example, Reading and multiple reading programs have demanded time that can only be obtained by making the day longer or stealing from other disciplines. We must educated the whole child in all the disciplines and be aware that no one subject exists in a sterile environment without others, these subject area demands needs, and skills.

- The main problem is time. My staff goes beyond the required hours the county and state mandate. Today, more staff members are requiring pay to attend any extra developments. This is fair, and the days of you do it for the kids is ending outside the structured day. Our staff is tired at the end of an instructional day and has families of their own to attend too. I'm happy that we are tired at the end of each day. The effort and dedication shows as the staff exits the building. We always have informal conversations about our day as each staff member leaves.

- The main problem is time. My staff goes beyond the required hours the county and state mandate. Today, more staff members are requiring pay to attend any extra developments. This is fair, and the days of you do it for the kids is ending outside the structured day. Our staff is tired at the end of an instructional day and has families of their own to attend too. I'm happy that we are tired at the end of each day. The effort and dedication shows as the staff exits the building. We always have informal conversations about our day as each staff member leaves.

- As always, time is a huge factor. Also monies are a concern.

- TIME PD

- Providing the time 
Appendix $\mathrm{J}$

Narrative Question 2: Non-PDS (P. 3)

- The challenges in education today involve the calendar debate, the constant lack of time and sufficient resources, mandates from above without the money or personnel to support their implementation, and lack of parent support for many things that we in education are forced to do.

- The teachers have too much to do. we cannot expect then to serve on committees and work in the evenings every weeknight.

- time

- Time and availability of courses/seminars, etc.

- Time for staff collaboration

\section{Training}

- Relevant training.

- The county should provide professional development for administrators that are pertinent to our needs.

- Fighting the Central Office in scheduling and maintaining collaboration time as well as trying to convince Central Office that our staff is well read, attends pertinent professional development activities, and is well versed in research.

- Providing the "culture" isn't difficult for the instructional leader, however the greatest challenges come with providing a professional development is, as is always the case in education, time, and money. The few days provided by the state/county for PD, typically held before school begins, don't address needs that develop during the year. Teachers' time is very valuable to them and they are cautious about committing to PD beyond the minimum requirements. Reading First got it right when they required teachers in their schools to complete 100 hours of PD annually. Also, with constant progress monitoring of students, they were able to focus PD on areas of need.

- I wish we were a PD school, but we attempt to grow professionally by conducting professional book studies, providing PD session with the Instructional Coaches or with teacher leaders within the school and visiting other schools. Our goal is to become a model school for advancing student achievement.

- On the clock, time to provide professional renewal with teacher leaders identified to lead vertical teams to meet school identified needs. Professional development needs to take 
Appendix $\mathrm{J}$

Narrative Question 2: Non-PDS (P. 4)

- On a more important meaning. It needs to not only provide expert, researched based staff development, it needs to include follow up with feedback and have an expectation that the staff development will be implemented.

- Adequate time to provide sustained Professional Development.

- Making sure professional development is valuable, relevant and of interest to all concerned

\section{Apathy and commitment by teachers}

- Getting all stakeholders to see the need for change and to motivate them to actually change.

- Work ethics

- Teachers willing to change their instruction to meet the needs of their students. Administrations willing to give resources needed. Time!!

- Apathy and disinterest among parents who have students with learning needs. Accountability of parents for assisting their child with appropriate activities that will maximize their success at school.

- Teachers must clearly see the connect with PD and practice. Talented professionals are not quick to give up successful proven practice for the newest idea unless it can be shown that it might be better and more effective than what they know and use.

- Teacher apathy.

- Challenges to professional renewal appear to be a plethora of "programs" and "quick fixes" as well as a natural reticence on the part of teachers to welcome change. Staff development must be pervasive and sustained within the school rather than a long series of "drive by" professional development episodes, which lack the necessary emphasis to become a vital part of the school culture.

- Teachers who believe "I have always taught this way and will continue to teach this way. It is not my fault if the students do not want to learn."

- The biggest challenge as an administrator is trying to adjust instructional practices according to the students of today. Many educators are comfortable with their current teaching style/strategies. The strategies work and they do not want to change. Slowly adding scientific based strategies to the current bank of information is the route I have been following. Changing the mind frame that there is no other way to teach is difficult. 
Appendix $\mathrm{J}$

Narrative Question 2: Non-PDS (P. 5)

\section{Direction}

- 1. Lack of time inside employment term and work hours to provide training. Staff is expected to attend training on their own time. 2. Curriculum and and instructional strategies are dictated by the state e.g., RTI (3 Tier Reading and Math). 3. Frequent state leadership shifts in paradigms affecting curriculum and instruction that require educators to drop previously adopted practices to learn new ones. 4. Staff morale problems resulting from increased demands without compensation and time to implement imposed changes.

- I am not sure.

- I taught in a PDS school for 14 years, working intensely with student teachers and their program. It affected my vision immensely. Access to current research and expectations, and working with the University to align best practices within the school and with the program was joyful hard work. This is my first job as a principal, and I miss being in a PDS. It is like the staff hasn't kept up... there's a definite time warp with technology and vision and best practices. The staff emphasized quiet and clean. Students in rows working on worksheets, occasionally standing up to recite an answer. It's been a long struggle.

- Small counties must unite because of student movement population and as the saying goes, "everyone must be on the same page." I have seen the cycles in instruction, when a school system finds something that works and results have been seen at the next school level leave it alone. Changing reading and math every few years is a major problem and it leaves gaps in a students learning. Professional development is necessary for new teachers but older teachers need to know what changes are happening in education so that their teaching method can be adjusted, such as when computers were introduced and new teaching tool become available.

- instill in administrators that they are there for the students first and it is not a job, it's a career

- $\mathrm{n} / \mathrm{a}$

- not sure

- We are not PDS but I have been a principal of a PDS school in the past. Communicating that all involved (student teacher, cooperating teacher, school administrator and higher ed supervisor) are all leaders and learners from day 1 observation block to retirement. Also establishing a strong and consistent orientation/plan at the cooperating school.

- Lack of clear understanding of purpose and beliefs. 


\section{Appendix J \\ Narrative Question 2: Non-PDS (P. 6)}

- Building an infrastructure that give teachers time and purpose to learn and collaborate with one another.

- Working with the Higher Education institution. We have approached Shepherd University about a PDS relationship, which they have agreed to. But all that it has produced is to have them send students here as a part of their Pedagogy coursework. 
Appendix K

Narrative Question 2: PDS (P. 1)

Complete listing of all responses from the experimental group made to Question 2(PDS)

What do you believe are the greatest challenges for providing a culture of professional renewal inside a Professional Development School system?

\section{Funding}

- lack of time and money

- Lack of funds to pay teachers to get renewed, school calendar, teachers recognizing the need for renewal.

- Having the resources to meet the challenges.

- Money to stipend staff and time CPR PD. Our county is very active with PD and when we add ours in it can overwhelm our teachers. We do have a great relationship with our higher Ed partner and provide their students with PD and experiences.

\section{Time}

- Time, too much high stake testing, state is stressing technology but no funding to provide it, too many top down guidelines, parental apathy

- Time for collaboration.

- Time

- Time...time to collaborate and build a culture of support and sharing is not available to teachers.

- Time for collaboration

- Time, space, funding.

- Time

- Time is always the biggest challenge. Providing your staff, time to have professional renewal is a key for it to happen. Teachers value time, and when it is provided to them, it shows the importance of the renewal effort.

- Time, money

- Time. The day is restricted by some much-required work that teachers have no time to use common sense based decisions.

- Time for renewal is the greatest inhibiter. Next is money for quality in-service. As a PDS, we were able to obtain funding for several years to hold grass roots PD based upon staff needs and desires. 
Appendix K

Narrative Question 2: PDS (P. 2)

- The constraints of time and money will continue to be the greatest of our challenges.

- Creating opportunities (time) for ongoing/imbedded staff development!

- Time and money

- There is not enough time built into the system for sustained professional development to take place. We don't need money to bring "big name" presenters in to our staff, we need the time to work with our staff.

- Time and resources are the greatest challenges.

- scheduling common planning not enough hours in the day

- Teacher Time to collaborate Teacher/Principal time to discuss best practices

- The greatest challenge is common time for sharing and financial resources to acquire training and pay teachers.

- The present system of scheduling and time constraints creates obstacles for opportunities of renewal inside a professional development school system.

- The time necessary to foster, grow, and adapt the PLC.

- Time and energy.

- Time and money

- Time constraints

- Time for collaboration

- we need more time to collaborate with each other, currently, meeting 5 days per year is not enough

\section{Training}

- Differentiated instruction should be stressed in the beginning.

- The greatest challenge I see is finding that very delicate balance of meeting the needs of the pre-service teachers, the classroom teachers, and the classroom students without overwhelming any one of these groups.

- Professional renewal should be based on ongoing, job embedded, and scientifically proven professional development.

- Provide appropriate professional development, time, and money for training.

- Willingness of every staff member to embrace being a life-long learner and their selfmotivation to eagerly want to improve professionally

- Staff recruitment and retention. High turnover and mobility among staff. Lack of time for professional development and collaboration. Lack of resources.

- The replacement of master teachers must occur and the technology applications must be implemented to address 21 st Century Learning. 
Appendix K

Narrative Question 2: PDS (P. 3)

- Choosing the area of importance that has the most impact on student learning. Many times personal agendas get in the way of our central core which is student learning.

- Staff eagerness to be lifelong learners. This is only the case with two of my staff members but nonetheless it is a challenge for me because I want/need $100 \%$ buy in to improve our school to the maximum level. Principals need legislative support in requiring teachers to grow professionally and not limiting professional development to 18 hours.

- The problems lie in jumping on every new trend with the belief that it will make all the difference in the world whether that is technology or some new strategy. It all comes down to teachers and the relationships they build with students. If it is positive, then there is a potential to learn. If the teacher is committed to the students learning, the learning occurs, no matter what ability or grade level a person teaches.

\section{Apathy and commitment by teachers}

- Overcoming resistance when implementing new changes.

- Commitment of new teachers to this as well as the older well established teachers.

- Not being able to actively evaluate those who don't have the best interests of their students at heart. I feel this is a Wood County problem from the administration at the top being influenced especially by WVAFT.

- Change never happens easily.

- The greatest challenge is that of providing and expecting ALL professionals to the same standards. Many seasoned professionals want to continue to instruct in the same manner as they have always instructed. It was good enough 30 years ago, so it should be good enough today.

- GETTING VETERAN TEACHERS TO MAKE CHANGES.

- I believe that the hardest thing to change about a school is the culture. What is important, what does the school hold important, etc. There is a difference between a faculty and a community of professional educators. For professionals to identify needs, to communicate these needs and share these ideas, and to address these needs are critical. The biggest challenge is "ourselves". But, with communication, collaboration, trust, etc. this can be addressed, nurtured and overcome...for the benefit of ALL of our Students.

- Maintaining the enthusiasm for the venture, and developing quality teacher leaders. 
Appendix K

Narrative Question 2: PDS (P. 4)

- We are a Marshall University Professional Development School. This has been a rewarding experience in that MU has provided us with wonderful resources. The greatest challenge is getting all staff onboard.

- All within a P.D.S. must be open-minded. They need the capacity to analyze situations and programs. They need to have a willingness to "tweek" and change to get "resultsfocused learning" for every student.

\section{Direction}

- Professional development must meet the needs of the staff, school, and what change is needed. It must bring something to the teachers more than filling a required time element.

- Getting everyone on the same page

- Federal and State oversight and regulations.

- Getting everyone to work together for the common good.

- Having teachers clearly understand the purpose and provide follow up.

- we are controlled by the results of assessments

- Complacency, cynicism, and Central Office

- A Professional Development School must be a "model school." I believe the collaboration can be profitable for both partners and the ultimate benefits can be seen not only in our school faculty and staff, our partner, but also in our students. I firmly believe that the professionals in the school must take ownership of their professional development and therefore, the schools becoming a "community of learners" where persons are permitted to set their own goals are most beneficial.

- We have to be considered and treated like professionals. The educational community as a whole is considered to be untrustworthy bums who have the summers off and do not work when it snows. 
Appendix L

Keywords and Concepts among the Standards of the ISLLC and NCATE

\begin{tabular}{|c|c|c|}
\hline Key Words and Concepts & $\begin{array}{c}\text { Interstate School Leaders } \\
\text { Licensure Consortium (ISLLC) } \\
\text { Standards }\end{array}$ & $\begin{array}{c}\text { National Council } \\
\text { Accreditation for } \\
\text { Accreditation of Teacher } \\
\text { Education (NCATE) }\end{array}$ \\
\hline Collaboration & Standard 1 and 2 & Standard 3 \\
\hline Leader or Leadership & Standards $1,2,3,4,5$, and 6 & Standard 5 \\
\hline Community & Standards 4 and 6 & Standard $1,2,4$, and 5 \\
\hline Success & Standards $1,2,3,4,5$, and 6 & Standard 4 \\
\hline Development & Standard 1 & Standards 1,4 , and 5 \\
\hline Vision & Standard 1 & Standards 1 and 5 \\
\hline Evaluate & Standards $1,2,3$, and 5 & Standards 2,4 , and 5 \\
\hline
\end{tabular}

